\title{
Structural and functional elucidation of the primary transducer module of the B cell antigen receptor
}

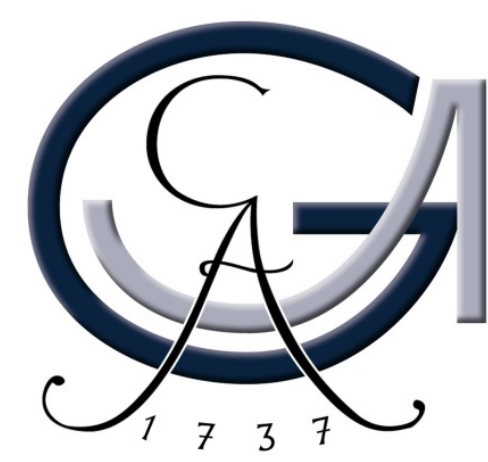

Dissertation

for the award of the degree

"Doctor rerum naturalium"

of the Georg-August Universität Göttingen,

within the doctoral program Molecular Biology

of the Georg-August University School of Science (GAUSS)

submitted by

Sona Pirkuliyeva

from

Ashgabat, Turkmenistan

Göttingen, 2015 


\section{Thesis committee}

Prof. Dr. Jürgen Wienands, University Medical Center Göttingen, Department of Cellular and Molecular Immunology

Prof. Dr. Lutz Walter, German Primate Center, Department of Primate Genetics

Prof. Dr. Christian Griesinger, Max-Planck Institute for Biophysical Chemistry, Department of NMR-based Structural Biology

\section{Members of the Examination Board}

Referee: Prof. Dr. Jürgen Wienands, University Medical Center Göttingen, Department of Cellular and Molecular Immunology

$2^{\text {nd }}$ referee: Prof. Dr. Lutz Walter, German Primate Center, Department of Primate Genetics

\section{Further members of the Examination Board}

Prof. Dr. Matthias Dobbelstein, University Medical Center Göttingen, Institute of Molecular Oncology

Prof. Dr. Blanche Schwappach, University Medical Center Göttingen, Department of Molecular Biology

Prof. Dr. Michael Thumm, University Medical Center Göttingen, Department of Cellular Biochemistry

Dr. Dieter Klopfenstein, Third Institute of Physics, Department of Biophysics

Date of the oral examination: $16^{\text {th }}$ of February, 2015 


\section{The declaration of academic honesty}

Herewith, I declare that I prepared the doctoral thesis "Structural and functional elucidation of the primary transducer module of the B cell antigen receptor" on my own, using only the sources cited and with the help of the persons as indicated.

Göttingen, 15th of January 2015

Sona Pirkuliyeva 


\section{Table of Contents}

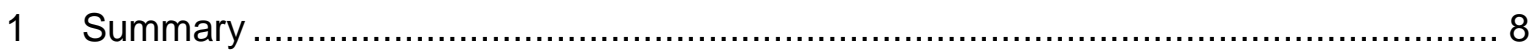

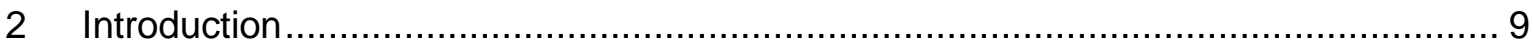

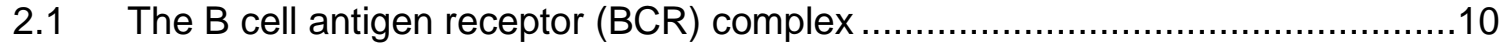

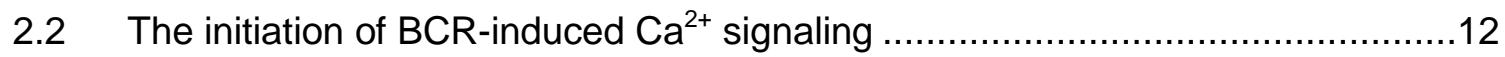

2.3 BCR-induced downstream signal transduction pathways and B cell fate ...........13

2.4 The role of the adaptor protein SLP65 in B cell activation ..............................16

2.5 The membrane recruitment process of SLP65 ............................................17

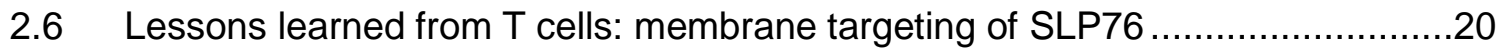

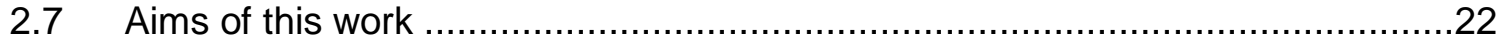

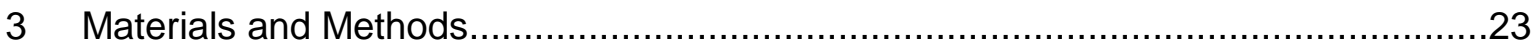

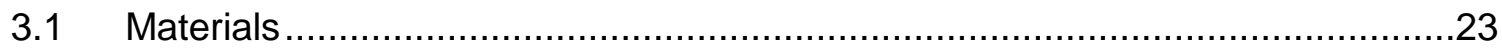

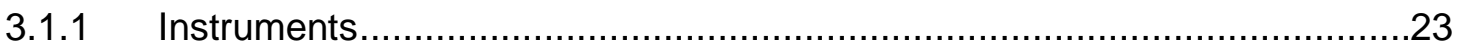

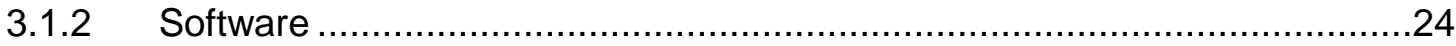

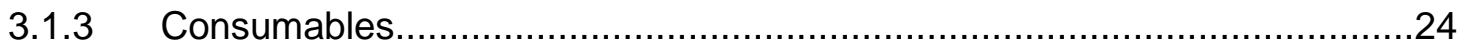

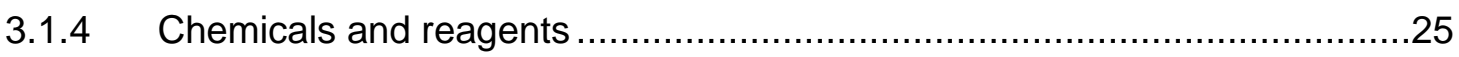

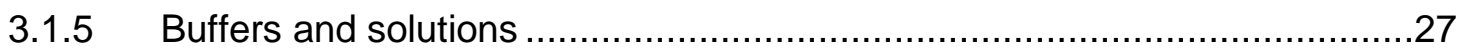

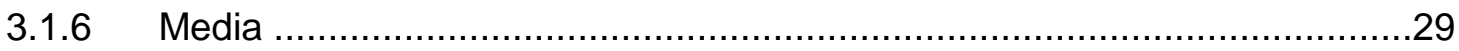

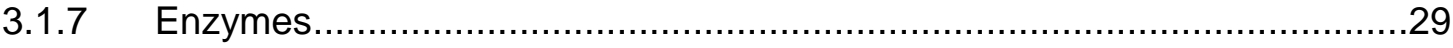

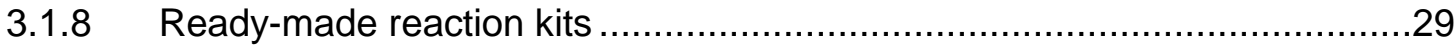

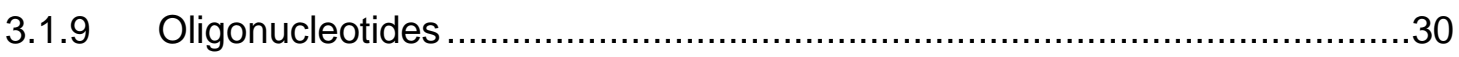

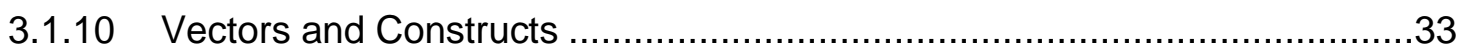

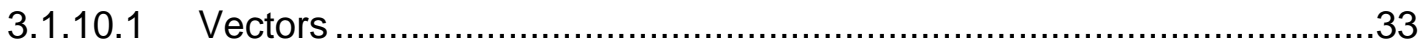

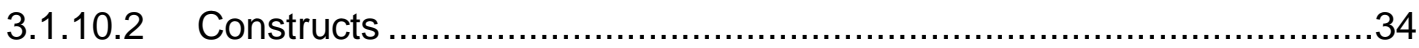

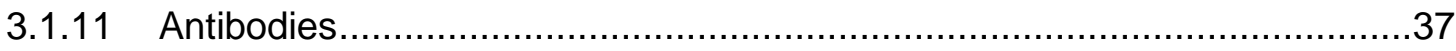

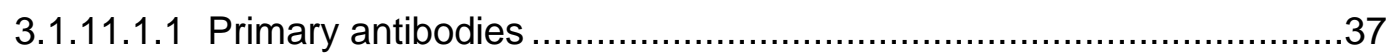




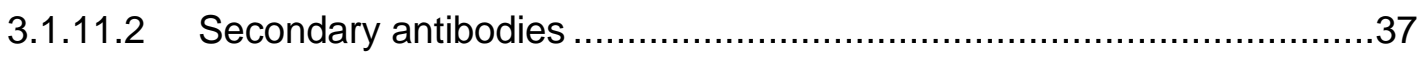

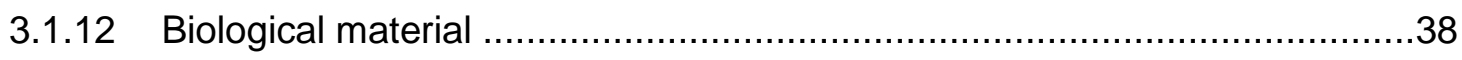

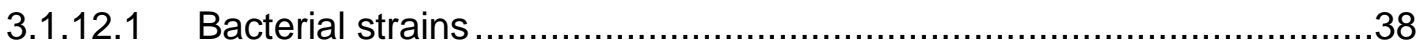

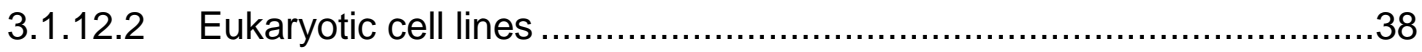

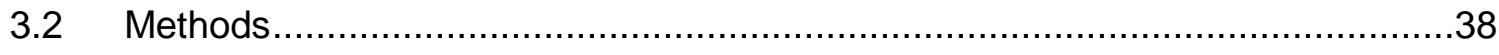

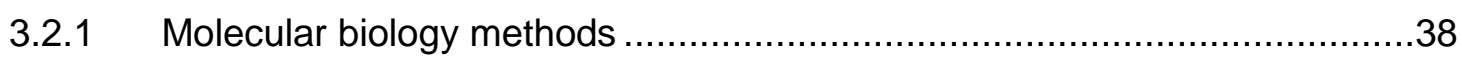

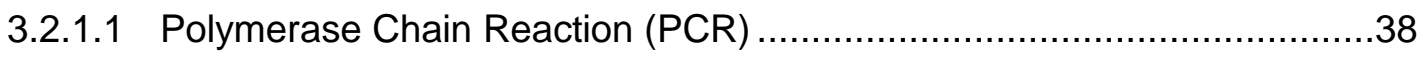

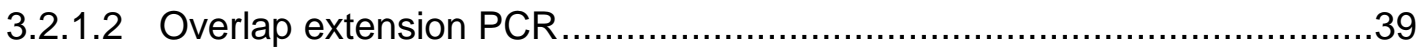

3.2.1.3 Site directed mutagenesis ....................................................... 40

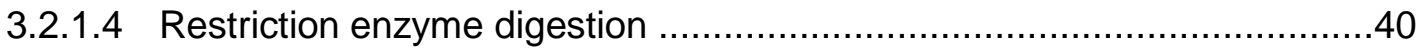

3.2.1.5 Agarose gel electrophoresis ................................................... 40

3.2.1.6 Purification and gel extraction of DNA fragments ............................41

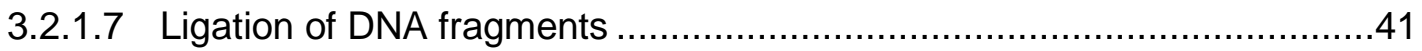

3.2.1.8 Cloning of PCR fragments via T/A cloning …...............................41

3.2.1.9 Transformation of chemo-competent E.coli ...................................... 41

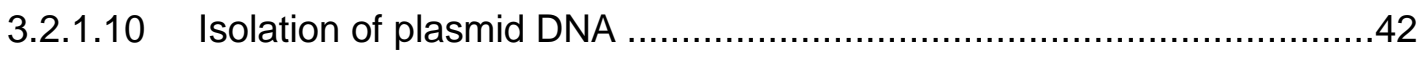

3.2.1.11 Total RNA isolation ...........................................................42

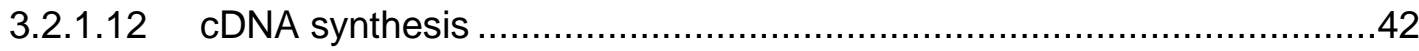

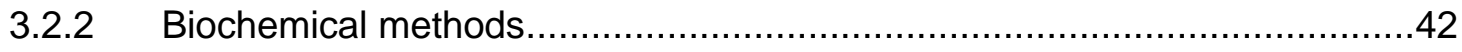

3.2.2.1 Expression and purification of recombinant GST proteins ....................42

3.2.2.2 Expression and purification of recombinant His-tagged proteins ............43

3.2.2.3 Photometric determination of protein concentration............................43

3.2.2.4 Preparation of cleared cellular lysates (CCLs) ..................................43

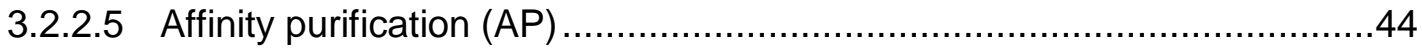

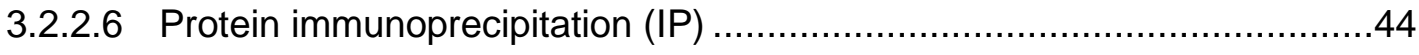

3.2.2.7 SDS polyacrylamide gel electrophoresis (SDS-PAGE) .......................44 


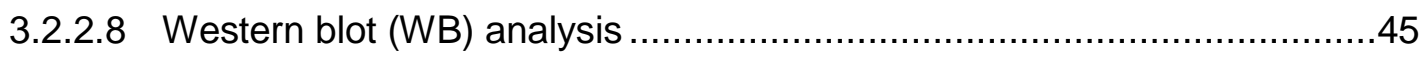

3.2.2.9 Preparation of SUVs by detergent exclusion .................................45

3.2.2.10 Preparation of LUVs by extrusion .............................................46

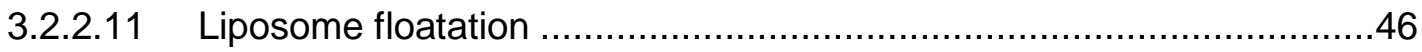

3.2.2.12 Preparation of SUVs for NMR and CD spectroscopy ......................46

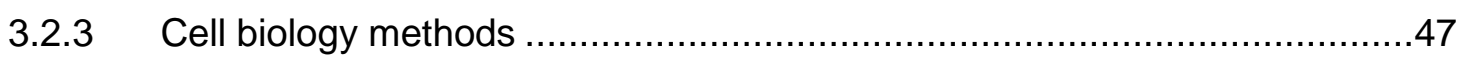

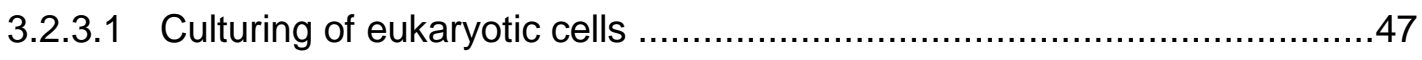

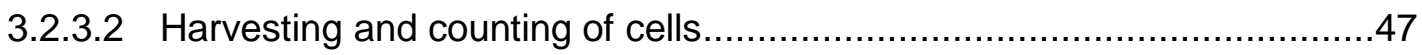

3.2.3.3 Freezing and thawing of cells .................................................... 47

3.2.3.4 Isolation of primary splenic mouse B cells ........................................

3.2.3.5 Transfection of PlatE cells by lipofection ..................................... 48

3.2.3.6 Retroviral transduction of DT40 and DG75 cells..............................48

3.2.3.7 Retroviral transduction of primary mouse B cells ...............................48

3.2.3.8 Analysis of $\mathrm{Ca}^{2+}$ flux by flow cytometry ......................................49

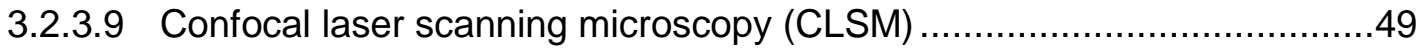

3.2.3.10 Colocalization analysis of fluorescence signals..............................50

3.2.3.11 Fluorescence recovery after photobleaching (FRAP) analysis .............50

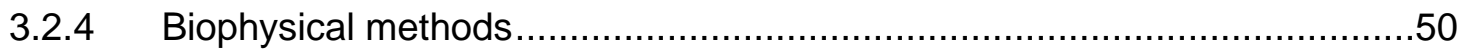

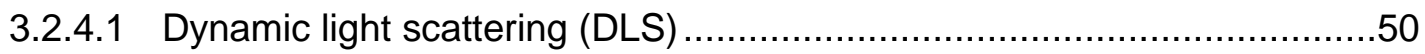

3.2.4.2 Circular dichroism spectroscopy............................................. 51

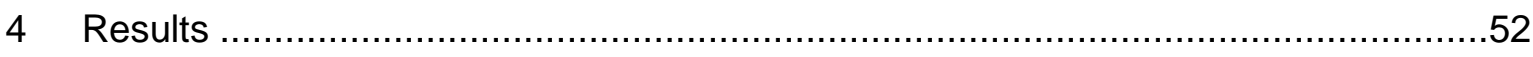

4.1 NMR analysis of the secondary structure of SLP65 and validation of the

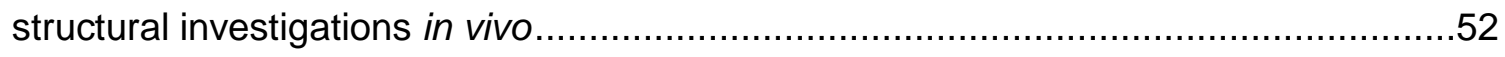

4.1.1 SLP65 possesses large intrinsically disordered regions .........................52

4.1.2 Intrinsic disorder in SLP65 is sufficient for the assembly of the $\mathrm{Ca}^{2+}$ initiation complex, but not for membrane targeting of SLP65 .......................................53

4.1.3 Btk and PLCY2 assemble on SLP65 in a spatially defined manner ............58 
4.2 Structural and functional analysis of the N-terminal region of SLP65

4.2.1 Evaluation of previous studies on the SLP65 $\mathrm{N}$ terminus.

4.2.2 The $\mathrm{N}$ terminus of SLP65 is a novel lipid-binding module which targets SLP65 to vesicles in resting $B$ cells

. .66

4.2.3 The $\mathrm{N}$ terminus of SLP65 binds preferentially to negatively charged membranes and may recognize membrane curvature

4.2.4 The CC domain of CIN85 is a lipid-binding domain, which preferentially binds to phosphatidic acid.

4.2.5 The SLP65 $\mathrm{N}$ terminus is disordered in solution, but binds to liposomes in a defined manner

4.2.6 Individual hydrophobic residues in the $\mathrm{N}$ terminus are essential for SLP65 function, but dispensable for lipid binding

4.2.7 The $\mathrm{N}$ terminus plays a differential role for SLP65 membrane targeting and $\mathrm{Ca}^{2+}$ signaling in different species and at different stages of $\mathrm{B}$ cell development ......89

4.2.8 The SLP65 N terminus depends on the remaining SLP65 sequence for full functionality. .93

5 Discussion 96

5.1 Intrinsic disorder in SLP65 and its implications for SLP65 function .96

5.2 The role of the SLP65 N terminus in the membrane targeting of SLP65 .98

5.3 The proposed mechanism used by the SLP65 $\mathrm{N}$ terminus for membrane anchoring .101

5.4 The $\mathrm{N}$ terminus cooperates with CIN85 for the efficient membrane targeting of SLP65 104

5.5 The vesicular route for the plasma membrane targeting of signaling proteins in lymphocytes 106

5.6 Conclusions and perspectives 109

6 Bibliography .111

7 Appendix. 128

7.1 List of Figures. 128 


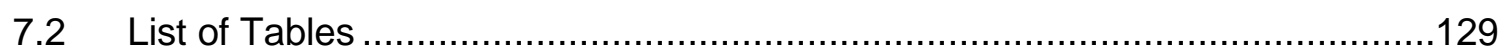

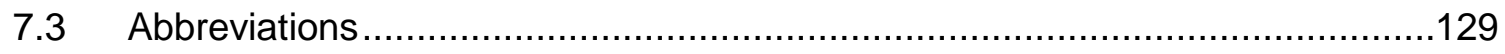

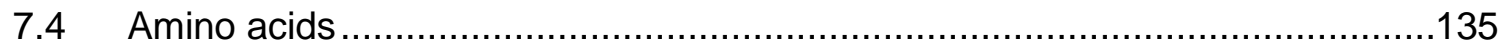

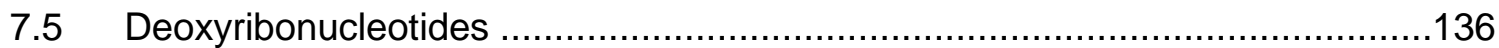

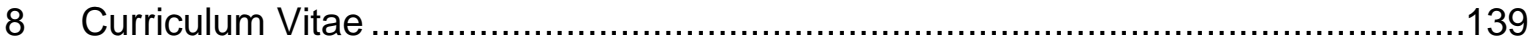




\section{Summary}

Interaction of an antigen with the $\mathrm{B}$ cell antigen receptor (BCR) initiates signaling cascades leading to $\mathrm{B}$ cell activation, proliferation and differentiation. Signal transduction downstream of the BCR depends on the adaptor protein SLP65 (SH2 domain-containing leukocyte adaptor protein of $65 \mathrm{kDa}$ ). Upon BCR activation, SLP65 translocates from the cytosol to the plasma membrane via a poorly understood mechanism, and recruits the enzymes Btk (Bruton's tyrosine kinase) and PLCү2 (Phospholipase C- $\mathrm{2} 2$ ), necessary for the release of the key secondary messenger $\mathrm{Ca}^{2+}$. As recently shown by our group, the pre-formed complex of SLP65 with CIN85 (Cbl-interacting protein of $85 \mathrm{kDa}$ ) is indispensable for efficient BCR signaling initiation (Oellerich et al., 2011). For my dissertation, I investigated the plasma membrane targeting process of this so called "primary BCR transducer module". Using imaging and biochemical assays, my colleagues and I showed that signaling-competent SLP65 is distributed between the cytosol and intracellular exosome-like vesicles in resting DT40 cells. Vesicular localization of SLP65 depended on its positively charged amino (N)-terminal region, encompassing 50 amino acids. I found that the $\mathrm{N}$ terminus of SLP65 is a direct lipid-binding module, which preferentially binds to negatively charged and curved membranes in vitro. In vivo, the $\mathrm{N}$ terminus of SLP65 could be functionally substituted by the curvature-sensitive lipidbinding N-BAR domain of Amphiphysin. However, lipid binding by the $\mathrm{N}$ terminus or the BAR domain was insufficient to target SLP65 to vesicles in the absence of CIN85 binding, indicating that the $\mathrm{N}$ terminus and CIN85 cooperate for precise subcellular targeting of SLP65. The finding that pre-assembly of signaling adaptors on vesicles is required for $B$ cell activation indicates that these signaling complexes may be delivered to the BCR via vesicular transport. Analysis of the secondary structure of SLP65 by Nuclear Magnetic Resonance (NMR) spectroscopy, in collaboration with the group of Prof. Christian Griesinger, showed that the N terminus of SLP65 is intrinsically disordered in solution, as well as upon lipid binding. Large regions of intrinsic disorder were also observed in the central part of SLP65. I investigated the functional role of intrinsic disorder in this region by substituting it with a flexible GS linker, or by rearranging the Btk- and PLCY2- binding motifs. My results indicate that secondary structure in this region is dispensable for the assembly of the $\mathrm{Ca}^{2+}$ initiation complex, but might be required for the membrane targeting of SLP65 via the vesicular route. Altogether, my studies contribute to the understanding of the membrane targeting process of the adaptor protein SLP65 and the BCR signaling initiation. 


\section{Introduction}

The immune system has evolved to protect a host organism from pathogens. Immune responses can be broadly classified as innate and adaptive. Innate immune response occurs rapidly upon exposure to a pathogen and is mediated by protective physical and chemical barriers such as mucosal linings and antimicrobial proteins, which prevent the spread of infection; and by specialized white blood cells such as neutrophils and macrophages, which engulf the pathogens and destroy them. The receptors of innate immune cells mediating this recognition are encoded in the germline of an organism and have broad specificity for repetitive molecular patterns on the pathogen's surface. Adaptive immune response, on the other hand, takes time to develop and is mediated by $B$ and $T$ lymphocytes, specialized immune cells, which have the ability to recognize and respond to individual antigens. $T$ lymphocytes mediate the cellular immune response they activate other cells of the immune system and stimulate them to produce cytokines that help to fight the infection. B lymphocytes mediate the humoral immune response they differentiate into plasma cells and produce antibodies, which bind to pathogens and together with the complement system and phagocytotic cells contribute to pathogen elimination. Antigen receptors of the adaptive immune system are generated throughout the individual's life time by the process of somatic recombination, and the receptors of $B$ cells are also further diversified upon encounter with the antigen by somatic hypermutation. As a result of these mechanisms, each lymphocyte expresses a unique receptor of defined antigen specificity on its cell surface. A population of lymphocytes therefore expresses a vast repertoire of receptors, able to respond to virtually any kind of antigen (Murphy, 2011).

The first step in B cell activation is the interaction of a B cell antigen receptor (BCR) at the cell surface with its cognate antigen. This activates signaling cascades inside the cell, which lead to proliferation and differentiation of immunocompetent $B$ cells or apoptosis of autoreactive $B$ cells. Following antigen binding, the BCR-antigen complex gets internalized and processed inside the endosomal compartment. The antigenic peptides can then be presented on the major histocompatibiliy complex (MHC) class II molecules at the cell surface to activated $T$ cells of similar antigen specificity. However, the specific peptide recognized by helper $T$ cell may be different from the protein epitope recognized by the BCR. T cells interact with the MHC molecules via their $\mathrm{T}$ cell antigen receptors (TCRs) and provide co-stimulatory signals to the B cell, necessary for full activation. The interaction between $B$ and $T$ cells takes place in the peripheral lymphoid tissues where 
they occupy different areas, but migrate towards the same location upon activation by the antigen. Following activation by helper T cells, B cells may form germinal centers in the lymphoid organs, which are the sites of intense B cell proliferation and differentiation. Activated B cells may differentiate into antibody producing plasma cells or long-lived memory B cells, which persist in the organism's lymphoid tissues and mediate secondary immune response upon reinfection with the same pathogen (Murphy, 2011).

\subsection{The B cell antigen receptor (BCR) complex}

The BCR complex consists of a membrane bound immunoglobulin molecule $(\mathrm{mlg})$, acting as the antigen recognition component, and a non-covalently associated disulfide-linked $\lg \alpha(\mathrm{CD} 79 \mathrm{a})-\lg \beta(\mathrm{CD79b})$ heterodimer, serving as the signal transducing component (Reth, 1992; Pleiman et al., 1994). The mlg molecule consists of two heavy chains, cross-linked via disulfide bonds, and two light chains, each covalently bound to a heavy chain. At the $\mathrm{N}$-terminus both heavy and light chains carry a variable $(\mathrm{V})$ domain, which binds antigen; at the $C$ terminus they possess a constant (C) domain, which binds to FC and complement receptors (Williams \& Barclay, 1988; Schroeder \& Cavacini, 2010).

The variable domains are generated by somatic recombination, a gene rearrangement event where $V(D) J$ gene segments are joined together to produce a $V$ region with $a$ defined antigen specificity (Tonegawa, 1983); and are diversified by somatic hypermutation, a process whereby mutations increasing the affinity of the $\mathrm{B}$ cell receptor for the antigen are introduced (Di Noia \& Neuberger, 2007). The C domain of the heavy chain $\left(\mathrm{C}_{\mathrm{H}}\right)$ determines the antibody isotype, which can be either $\lg \mathrm{M}, \lg \mathrm{G}, \lg \mathrm{D}, \lg \mathrm{E} \operatorname{or} \lg \mathrm{A}$, with each isotype having different effector functions (reviewed by Schroeder \& Cavacini, 2010). The $C_{H}$ gene is joined to the $V(D) J$ region after the recombination of the latter has taken place. In naïve $B$ cells the $\mu$ gene of the constant region $(C \mu)$, located at the $V_{H}$ proximal end of the $\mathrm{C}_{\mathrm{H}}$ gene cluster, is expressed, thereby giving rise to an IgM type of BCR. After antigenic stimulation, an activated B cell may undergo class-switch recombination, a process which replaces the $\mathrm{C} \mu$ gene with $\mathrm{C} \gamma, \mathrm{C} \varepsilon$ or $\mathrm{C \alpha}$, resulting in a new isotype of antibody (reviewed by Honjo et al., 2002). The $C$ domain of the light chain $\left(C_{L}\right)$ can be of only two types $-\lambda$ and $k$, the functional differences of which are not known (Reth, 1992). The gene rearrangement processes in heavy and light chains are regulated by a mechanism known as allelic exclusion, which ensures that only one type of rearranged $\mathrm{IgH}$ and $\mathrm{Ig} \mathrm{L}$ is produced, giving rise to an $\mathrm{lg}$ molecule with two identical antigen-binding sites (for review, see Bergman \& Cedar, 2004). 
The cytoplasmic tails of Ig molecules are relatively short, therefore the signal-transducing component in form of Ig $\alpha$ and $\lg \beta$ is needed (Sanchez et al., 1993; Teh \& Neuberger, 1997). The cytoplasmic domains of $\lg \alpha$ and $\lg \beta$ contain the immunoreceptor tyrosinebased activation motifs (ITAMs) with a consensus sequence of D/Ex7D/ExxYxxLx7YYxxI/L (Reth, 1989). These motifs are essential for signal transduction, since they become tyrosine phosphorylated by the BCR-associated protein tyrosine kinases (PTKs) (Gold et al., 1991), and recruit Src-homology 2 (SH2) domain-containing cytosolic effector proteins (Flaswinkel \& Reth, 1994; Kurosaki et al., 1995).

The assembly of the functional BCR complex at the cell surface is a critical step for B cell development. The rearrangement of the heavy chain gene takes place first. The heavy chain starts to be expressed at the plasma membrane at the precursor (pre)-B cell stage, while the light chain remains in germline configuration. Instead of the conventional light chain, a complex of surrogate light chains $\lambda_{5}$ or $V_{\text {preB }}$ can associate with the heavy chain (Tsubata \& Reth, 1990) in pre-B cells. Signaling through this pre-BCR complex induces B cell proliferation and rearrangement of the light chain (Reth et al., 1987), allowing the developmental transition from pre-B cell stage to immature $B$ cells to take place (for review, see Melchers et al., 2000). Immature B cells migrate out of the bone marrow, where the initial steps of maturation take place, and enter the spleen, where they go through intermediate stages of maturation, known as T1 (transitional type 1) and T2 (transitional type 2), before they become mature B cells. The transitional B cell subsets differ in their location within the spleen, and in surface expression of some markers (reviewed by Chung et al., 2003). Following their exit from the bone marrow, transitional B cells, which encounter self-antigens in the periphery, are programmed for deletion by apoptosis or anergy (reviewed by Goodnow et al., 2010). B cell repertoire selection is regulated by the strength of $B C R$ signaling and by the interaction of transitional $B$ cells with survival factors, such as BAFF (B cell activation factor of the TNF family) (reviewed by Mackay et al., 2003). As T2 cells become mature B cells, they downregulate their surface $\lg \mathrm{M}$ and upregulate surface $\lg \mathrm{D}$ expression and move to the B-cell follicles in the spleen.

Pre-BCR/BCR signaling regulates $B$ cell fate at all developmental stages. The BCR signaling cascade starts with the interaction of the BCR with the antigen (or ligandindependent receptor clustering), and subsequently diverges into multiple branches, which operate at different stages of B cell development and determine cellular responses. 


\subsection{The initiation of BCR-induced $\mathrm{Ca}^{2+}$ signaling}

As mentioned above, interaction of an antigen with the BCR transmits signals to the interior of the cell leading to activation of PTKs and phosphorylation of ITAMs on Iga and $\lg \beta$. It is not exactly clear how antigen binding to the extracellular domain of the BCR induces these changes in the intracellular domains. It is thought that antigen binding induces a conformational change in the BCR structure; however, the nature of this conformational change remains to be elucidated. Both soluble and membrane bound antigens can activate B cells in vitro, even though it is believed that in vivo B cells are activated predominantly by membrane-bound antigens (Carrasco \& Batista, 2006). Moreover, it has been observed that monovalent antigens provided in solution cannot activate B cells (Landsteiner, 1924; Fanger et al., 1970; Feldmann, 1972; Minguet et al., 2010). Therefore it is thought that $B$ cell activation is mediated by multivalent antigens, which results in clustering of several BCR complexes at the cell surface upon antigen binding, known as the cross-linking model (Metzger, 1992). Upon cross-linking, the BCRs translocate into the sphingolipid and cholesterol-rich microdomains in the plasma membrane, known as lipid rafts (Cheng et al., 1999). According to an alternative model, several BCR molecules exist in oligomeric state on the cell surface, and antigen binding results in their dissociation, which is then translated to the intracellular signaling proteins (Schamel \& Reth, 2000; Reth, 2001).

Whatever the nature of the conformational change in the BCR structure is, it triggers the activation of several PTKs (Burkhardt et al., 1991; Gold et al., 1991). One of the earliest kinases to be activated is Lyn, a Src family tyrosine kinase, which is associated with the BCR complex and phosphorylates tyrosine residues in the ITAMs of Iga and $\lg \beta$ (Yamanashi et al., 1991; Campbell \& Sefton, 1992; Yamanashi et al., 1992; Yamamoto et al., 1993). Phosphorylation of ITAMs leads to the assembly of spatially resolved signaling complexes in the lipid rafts, sometimes referred to as micro-signalosomes (Treanor et al., 2009). The phosphorylated ITAMs serve as docking sites for the tandem $\mathrm{SH} 2$ domains of intracellular kinase Syk, thereby recruiting it to the BCR (Wienands et al., 1995; Futterer et al., 1998). Moreover, binding of Syk to phosphorylated Iga/ $\beta$ stimulates its kinase activity and results in Syk autophosphorylation (Rowley et al., 1995). This is related to relieving the inhibition by the tandem SH2 domains, which mask the catalytic domain in the resting state (Tsang et al., 2008). The substrate for Syk is adaptor protein SLP65 (SH2 domaincontaining leukocyte adaptor protein of $65 \mathrm{kDa}$ ), also known as BLNK (B-cell linker) or BASH (B-cell adaptor containing an SH2 domain) (Fu et al., 1998; Wienands et al., 1998). 
It is a cytosolic adaptor protein, which translocates to the plasma membrane and links the signals from BCR engagement to several intracellular effector proteins. SLP65 interacts with many components of the BCR signaling pathway, including Vav, Nck, Grb2 (for review, see Koretzky et al., 2006); but most importantly, it recruits and forms a complex with Btk and PLCy2 (Hashimoto et al., 1999; Ishiai et al., 1999b), which are enzymes, mediating the release of the key secondary messenger $\mathrm{Ca}^{2+}$ in $\mathrm{B}$ cells. In this trimolecular complex, known as the $\mathrm{Ca}^{2+}$ initiation complex, Btk comes in close proximity to PLCY2 and can phosphorylate and activate it (Takata \& Kurosaki, 1996). SLP65 therefore regulates both the assembly and the membrane targeting of this complex (Ishiai et al., 1999a; Su et al., 1999; Chiu et al., 2002; Engelke et al., 2007). Btk additionally uses its pleckstrinhomology $(\mathrm{PH})$ domain for membrane targeting, which binds phosphatidyl inositol triphosphate $\left(\mathrm{PI}(3,4,5) \mathrm{P}_{3}\right)$, a phospholipid generated by the action of the $\mathrm{PI}$ kinase upon activation of the BCR co-receptor CD19 (Scharenberg et al., 1998). Activated PLCY2 catalyses the hydrolysis of phosphatidyl inositol 4,5-bisphosphate $\left(\mathrm{PI}(4,5) \mathrm{P}_{2}\right)$ to secondary messengers inositol triphosphate $\left(\mathrm{IP}_{3}\right)$ and diacylglycerol (DAG) (Hempel et al., 1992; Kurosaki et al., 2000). $I P_{3}$ binds to $I_{3}$ receptors $\left(I P_{3} R s\right)$ in the membrane of the endoplasmic reticulum (ER), which are $\mathrm{Ca}^{2+}$ channels that open upon $\mathrm{IP}_{3}$ binding. This results in a flux of $\mathrm{Ca}^{2+}$ according to its gradient from ER to the cytosol (for review, see Patterson et al., 2004). Depletion of $\mathrm{Ca}^{2+}$ in the intracellular stores is sensed by STIM1, an ER resident transmembrane protein, which multimerizes and associates with store-operated $\mathrm{Ca}^{2+}$ channels (SOCs) in the plasma membrane, resulting in their activation (Baba et al., 2006). This leads to opening of these channels and $\mathrm{Ca}^{2+}$ influx from the extracellular space (for review, see Parekh \& Penner, 1997; Putney \& Ribeiro, 2000).

\subsection{BCR-induced downstream signal transduction pathways and B cell fate}

The secondary messengers generated by PLCY2 mediate activation of downstream signaling cascades: activation of mitogen-activated protein kinases (MAPKs), such as the extracellular signal-regulated kinase (ERK), the c-Jun N-terminal kinase (JNK) and the p38 kinase; and transcription factors, such as the nuclear factor of activated $\mathrm{T}$ cells (NFAT) and the nuclear factor-kB (NF-kB). These different signaling pathways regulate survival, proliferation, differentiation and apoptosis of developing B cells (for review, see Niiro \& Clark, 2002). Some of the pathways and their cellular outcomes are outlined below. 


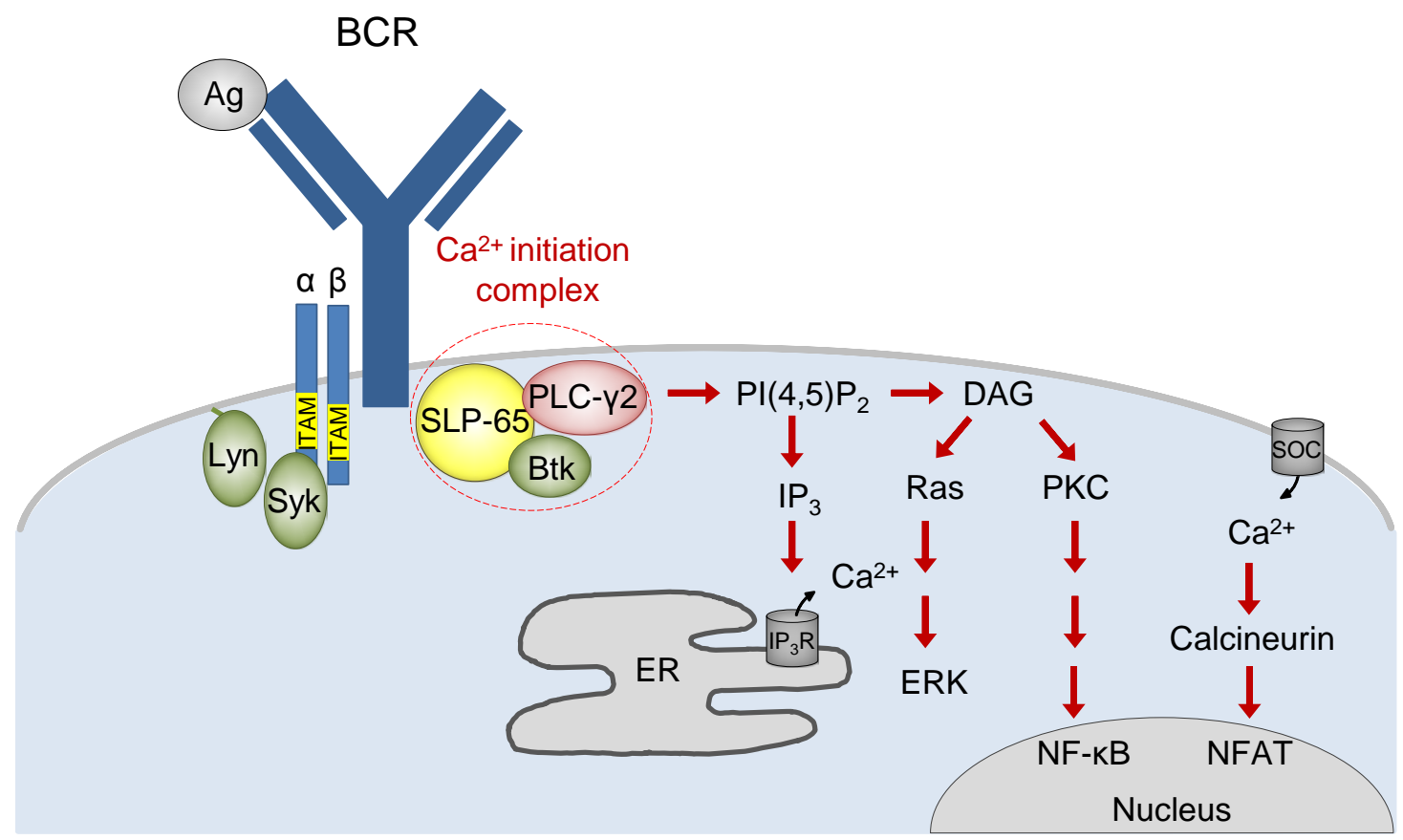

Figure 2.1 A simplified scheme of the key signaling events in the BCR signal transduction pathway.

Upon antigen binding BCR molecules on the cell surface are cross-linked (not shown) and the PTKs Lyn and Syk are activated. Lyn phosphorylates ITAM residues in Iga and Igß, and Syk is recruited to phosphorylated ITAMs. Syk phosphorylates adaptor protein SLP65, which acts as a scaffold for the assembly of the $\mathrm{Ca}^{2+}$ initiation complex, consisting of SLP65, Btk and PLCy2. Btk phosphorylates and activates PLCy2. Activated PLCY2 hydrolyses the membrane lipid $\mathrm{PI}(4,5) \mathrm{P}_{2}$ into secondary messengers $I_{3}$ and DAG. $I P_{3}$ binds to $I P_{3} R$ s at the ER membrane and initiates $\mathrm{Ca}^{2+}$ influx from the ER, followed by $\mathrm{Ca}^{2+}$ influx from the extracellular space. $\mathrm{Ca}^{2+}$ and DAG then activate downstream signaling pathways, such as the MAPK ERK, and the transcription factors NFAT and NF-KB.

\section{Ras/ERK pathway}

BCR engagement leads to plasma membrane recruitment and activation of small GTPase Ras. This is mediated by two mechanisms: via the Guanine nucleotide exchange factor (GEF) Sos and adaptor protein Grb2, which assemble at the activated BCR (Saxton et al., 1994), possibly recruited there via the interaction of Grb2 with SLP65 (Fu et al., 1998); and/or via another GEF for Ras, RasGRP3, which is recruited to the plasma membrane by means of its interaction with the PLCY2 product DAG (Oh-hora et al., 2003). Once activated, the GTP-bound Ras binds and activates Raf-1 and B-Raf, which phosphorylate and activate MEK1/MEK2, which in turn phosphorylate ERK1/ERK2. Phosphorylated ERKs dimerize and translocate to the nucleus, where they phosphorylate and thereby regulate the activity of transcription factors of the Fos and Jun family (for review, 
see Kurosaki et al., 2010). Erk kinases play important role in B cell development, since they have been demonstrated to be required for transition of pro-B cells to pre-B cells and pre-BCR mediated cell expansion (Yasuda et al., 2008). The ERK pathway is also important for the survival of mature B cells (Richards et al., 2001).

$P K C-\beta / N F-K B$ pathway

Another enzyme recruited to the plasma membrane via its interaction with the PLCY2 product DAG is the serine/threonine kinase PKC- $\beta$. It is activated by $\mathrm{Ca}^{2+}$ binding and phosphorylates the adaptor protein CARMA1 (Sommer et al., 2005). Phosphorylation of CARMA1 enables its association with two other adaptors Bcl10 and MALT1. This leads to the recruitment of kinase TAK1 to CARMA1, which phosphorylates the IKK complex (Shinohara et al., 2005). The activated IKK complex can then phosphorylate the inhibitor of NF-KB (IKB), leading to its dissociation from NF-KB (Saijo et al., 2002; Su et al., 2002; Shinohara et al., 2005). This leads to nuclear translocation of NF-KB, where it activates the transcription of NF-kB-regulated genes. NF-KB signaling is important for survival of $B$ cells, since genetic ablation of upstream activators of NF-KB in mice makes B cells prone to apoptosis (Kaisho et al., 2001). NF-KB target genes encode many different cytokines and chemokines, receptors for immune recognition, proteins involved in antigen presentation, etc., making the NF-kB family of transcription factors the central mediator of immune response (for review, see Pahl, 1999).

\section{Calcineurin/NFAT pathway}

Increased cytosolic $\mathrm{Ca}^{2+}$ concentration is also sensed by the key $\mathrm{Ca}^{2+}$ sensor Calmodulin, which activates several target molecules, including the serine/threonine phosphatase Calcineurin. Calcineurin dephosphorylates the transcription factor NFAT in the cytosol, which leads to its translocation to the nucleus and activation of NFAT-responsive genes (for review, see Crabtree \& Olson, 2002). B cells express three NFAT members, NFATc1, NFATc2 and NFATc3 (Timmerman et al., 1997). Even though mice deficient for each NFAT member have been described, the B cell-specific phenotype of NFAT deficiency has not been reported (Peng et al., 2001). As shown by the B cell-specific ablation of the regulatory subunit of Calcineurin, the Calcineurin/NFAT signaling pathway appears to be not essential for the development and maintenance of B cells, but necessary for plasma cell differentiation, suggesting that this signaling pathway has a modulatory role in B cell responses (Winslow et al., 2006). 


\subsection{The role of the adaptor protein SLP65 in B cell activation}

The signaling pathways downstream of the $\mathrm{B}$ cell antigen receptor need to be tightly regulated in order to control $B$ cell responses. Adaptor proteins play important roles in the regulation of signaling cascades. Even though they lack enzymatic activities, adaptor proteins can control localization and assembly of signaling complexes, thereby linking enzymes with their substrates. One such "signal integrator" is the adaptor protein SLP65. It transmits signals from the BCR to the cytosolic effector molecules and links the BCRactivated Syk kinase to downstream signaling pathways.

SLP65 is expressed at all stages of B cell development starting from the pro-B cell stage (Pappu et al., 1999). The functional importance of this protein has been demonstrated by a number of studies. The function of SLP65 was first analyzed in DT40 chicken B cell lymphoma line. SLP65-deficient DT40 cells show no increase in cytosolic $\mathrm{Ca}^{2+}$ concentration and fail to activate the $\mathrm{PLC} Y 2 / \mathrm{IP}_{3}$ pathway upon BCR stimulation. Loss of SLP65 also compromised ERK activation and abolished JNK and p38 activation in DT40 cells (Ishiai et al., 1999a). In mice, SLP65 deficiency results in an incomplete block of B cell development. An increased number of pre-B cells in the bone marrow, a reduced number of immature $B$ cells in the periphery, and a complete absence of mature $B$ cells were observed in s/p65\% mice (Jumaa et al., 1999). B cells of these mice are also reported to have strongly compromised $\mathrm{Ca}^{2+}$ mobilization and to be less-responsive to proliferation induced by B cell mitogens (Jumaa et al., 1999; Xu et al., 2000). Moreover, s/p $65^{-/}$mice show an impaired immune response to T-cell-independent, but not to T-celldependent antigens (Xu et al., 2000), and have strongly reduced serum levels of IgM, but not IgG antibodies (Jumaa et al., 1999). The mouse phenotype of SLP65 deficiency is similar to that of SLP65-deficient human patients, who exhibit a block in pro- to pre-B cell transition and have no mature B cells, resulting in immunodeficiency (Minegishi et al., 1999). Interestingly, the phenotypes of SLP65 deficiency in mice and human closely resemble that of $X$-linked agammaglobulinemia, a disease associated with the loss of Btk expression (Xu et al., 2000), indicating that SLP65 and Btk are involved in the same signaling pathway. However, since the inhibition of B cell function and development in SLP65 deficient mice and human patients is incomplete, it is likely that the role of SLP65 in $B$ cell activation is partially redundant, and it can be compensated for by other signaling elements.

The main function of SLP65 in BCR signaling is a scaffolding one; it orchestrates the assembly and membrane targeting of the $\mathrm{Ca}^{2+}$ initiation complex (SLP65/Btk/PLCY2), and 
recruits other effector proteins to the BCR. This role of SLP65 is dependent on its tyrosine-phosphorylation. SLP65 is phosphorylated at 5 tyrosine residues by Syk kinase Y72, Y84, Y96, Y178 and Y189 (positions correspond to human SLP65) (Chiu et al., 2002). Phosphorylation of SLP65 creates docking sites for $\mathrm{SH} 2$ domain-containing signaling proteins Vav (Y72), Nck (Y72), Btk (Y96) and PLCY2 (Y178 and Y189) (Fu et al., 1998; Hashimoto et al., 1999; Su et al., 1999). More recently, another phospho-tyrosine residue in SLP65, Y119, was reported and found to bind the C2 domain of PLCY2 (Engelke et al., 2013). As mentioned earlier, interaction of SLP65 with Btk and PLCy2 enables PLCY2 activation and $\mathrm{Ca}^{2+}$ mobilization. Interactions of SLP65 with Vav, Nck and Grb2 are believed to have regulatory effects on BCR signaling and link BCR ligation to cytoskeletal rearrangements and MAPK activation (Fu et al., 1998; Johmura et al., 2003; Oellerich et al., 2011). A mutant variant of SLP65 with $Y$ to $F$ substitutions in the 5 phosphorylation sites $\left(\mathrm{Y}_{\mathrm{all}} \mathrm{F}\right)$, making it unable to be phosphorylated by Syk, cannot activate PLCY2, and shows compromised $\mathrm{Ca}^{2+}$ and NFAT responses upon BCRstimulation. This indicates that phosphorylation of SLP65 bridges Syk activation to BCRdownstream signaling cascades (Fu et al., 1998). Interestingly, the phosphorylationdeficient and signaling-incompetent variant $Y_{\text {all }} F$ can still be recruited to the plasma membrane upon BCR stimulation. This indicates that phosphorylation of SLP65 is independent from and downstream of plasma membrane translocation (Goldbeck, 2007). Dephosphorylation of signaling effectors by BCR-associated phosphatases plays an important role in negative regulation of BCR signaling. Dephosphorylation of SLP65 is mediated by SHP1, which is recruited to the inhibitory co-receptor of BCR CD22 (Gerlach et al., 2003).

Even though the importance of SLP65 for BCR signaling and B cell activation in general has been long recognized, it is not fully comprehended how SLP65 communicates to the activated BCR. Several studies of our and other groups have contributed to the understanding of SLP65 activation and membrane recruitment process and are described in the next section(s).

\subsection{The membrane recruitment process of SLP65}

SLP65 is a multi-domain protein with an N-terminal positively charged region, followed by a central domain containing several proline-rich motifs and inducibly phosphorylated tyrosines, and a C-terminal SH2 domain (Figure 2.2 and Koretzky et al., 2006). 


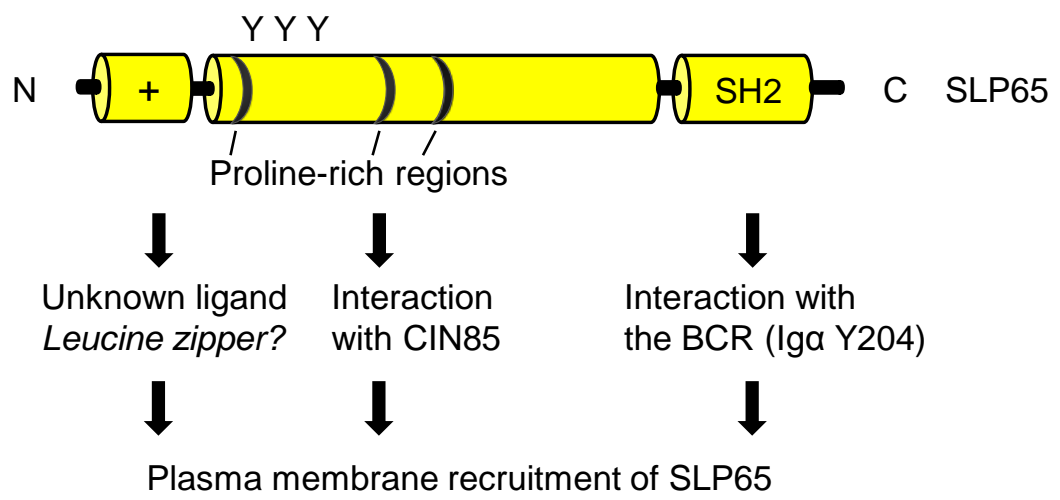

Figure 2.2 Domains and interaction partners of SLP65 involved in membrane targeting.

At the $\mathrm{N}$ terminus, SLP65 has a positively charged region, proposed to form a leucine zipper, the ligand of which is unknown. The central domain contains inducibly phosphorylated tyrosines and proline-rich regions, the latter mediating interaction with adaptor CIN85. The C-terminal SH2 domain binds to the phosphorylated Y204 in the BCR subunit Iga.

The N-terminal region of SLP65 contains several positively charged residues, such as lysines and arginines, interspersed by hydrophobic amino acids, such as leucines and isoleucines. Based on the amino acid sequence and mutational analysis, a leucine zipper motif has been proposed in the N terminus of SLP65. It was found to confer some degree of general membrane association to SLP65 in B cells and when ectopically expressed in other cell types. Amino acid exchanges of residues L18 and I25, inactivating the putative leucine zipper motif, have been reported to abolish the membrane association of SLP65 and the respective single point mutants couldn't relieve the developmental block when expressed in mouse s/p65 $5^{-/}$pre-B cells (Kohler et al., 2005). However, no ligand for the proposed leucine zipper motif in SLP65 $\mathrm{N}$ terminus was identified and the respective mechanism of SLP65 targeting remains elusive. N. Herrmann in our lab has investigated the potential interaction of the SLP65 N terminus with Calmodulin and PIP (phosphatidyl inositol phosphate) species in vitro (Herrmann, 2009). These interactions and their relevance for membrane recruitment of SLP65 need to be validated in vivo.

Another "hand" that SLP65 uses to anchor itself to the plasma membrane is its SH2 domain. It has been shown to associate with a phosphotyrosine residue located outside of the ITAM motif in Iga, Y204, upon BCR ligation. This interaction was proposed to mediate the recruitment of SLP65 to the activated BCR directly (Engels et al., 2001; Kabak et al., 2002). However, mice harboring the knock in mutation Y204F in their lga show only mild defects in BCR signaling and B cell maturation (Patterson et al., 2006). Moreover, experiments employing chimeras consisting of the mouse CD8 extracellular domain and 
the $\lg \alpha / \lg \beta$ cytoplasmic domain have indicated that SLP65 can also be recruited to the CD8-Ig $\beta$ chimera independent of Iga (Pike \& Ratcliffe, 2005). From these studies it was therefore concluded that the Y204-mediated recruitment of SLP65 is dispensable for SLP65 activation and rather amplifies signaling. Nevertheless, the $\mathrm{SH} 2$ domain is essential for SLP65 function. Mutant variants of SLP65 harboring an inactivating mutation within or lacking the $\mathrm{SH} 2$ domain completely fail to translocate to the plasma membrane and activate $\mathrm{Ca}^{2+}$ mobilization upon BCR engagement, when expressed in s/p65/- DT40 cells (Abudula et al., 2007). This indicates that an additional ligand for the SLP65 SH2 domain exists, which mediates membrane anchoring. An association of the SLP65 SH2 domain with Syk kinase was reported, however this interaction seems to be of relevance for a SLP65-downstream adaptor function of Syk, and not for the plasma membrane recruitment process of SLP65 (Abudula et al., 2007).

A quantitative mass spectrometry approach has been used by our group to map SLP65 interactions in real time. Numerous constitutive and inducible SLP65 ligands were identified. Moreover, the kinetics of their association with SLP65 was monitored, which allowed to distinguish between early and late binding partners. In this study, the protein $\mathrm{C}$ type lectin 17A (CLEC17A) was identified as one of the early and most dynamic SLP65 interaction partners in DT40 cells (Oellerich et al., 2011). CLEC17A was found to be phosphorylated upon BCR ligation and interact with the SH2 domain of SLP65 (Von Spee, 2010). In my Master's thesis project I investigated the role of CLEC17A in B cell activation and generated a clec17 $a^{-/-}$DT40 cell line. The BCR-proximal signaling events, such as the translocation of SLP65 to the plasma membrane and the $\mathrm{Ca}^{2+}$ mobilization, were not affected in CLEC17A-deficient cells, indicating that this protein is not essential for the membrane targeting of SLP65 (Pirkuliyeva, 2012). The missing link between the SLP65 $\mathrm{SH} 2$ domain and the BCR therefore still remains to be elucidated.

The interactome analysis of SLP65 has also identified another adaptor molecule, Cblinteracting protein of $85 \mathrm{kDa}$ (CIN85), as a constitutive interaction partner and critical helper for the membrane translocation of SLP65. The interaction between the two proteins was found to be mediated by the $3 \mathrm{SH} 3$ domains of CIN85 and 3 proline-rich motifs in SLP65. A mutant variant of SLP65 with inactivating mutations in the 2nd and 3rd prolinerich regions (SLP65 M23), making it unable to bind CIN85, could not efficiently translocate to the plasma membrane and support BCR-induced $\mathrm{Ca}^{2+}$ mobilization and NF-KB activation (Oellerich et al., 2011). Analysis of CIN85 localization by total internal reflection microscopy (TIRF) has shown that it colocalizes with the BCR-containing microclusters at 
the cell surface in DT40 cells, moreover this colocalization was independent of SLP65 (Oellerich et al., 2011; Bremes, 2012). In mice, B cell-specific ablation of CIN85 leads to defective NF-KB activation and reduced $\mathrm{T}$ cell-independent type II antibody responses (Kometani et al., 2011). However, the BCR-proximal responses, such as tyrosine phosphorylation and $\mathrm{Ca}^{2+}$ mobilization, are unimpaired. This can be attributed to the functional redundancy between CIN85 and CD2AP (CD2-associated protein), adaptor protein also expressed in B cells with similar domain architecture, which is able to partially substitute CIN85 function (Bremes, 2012). It is not clear how CIN85 aids membrane translocation of SLP65, or how the BCR-localization of CIN85 itself is achieved. Association of CIN85 with phosphatidic acid (PA), a lipid generated by the action of enzyme phospholipase D (PLD), has been reported (Zhang et al., 2009) and could contribute to the membrane localization of CIN85 in BCR-stimulated cells. Further investigation of the subcellular localization of CIN85 and its interaction partners in B cells is needed to comprehend its role in BCR signaling and SLP65 activation.

Collectively, the studies on membrane recruitment of SLP65 have shown that it is a complex process, requiring cooperation of multiple domains and interaction partners of SLP65.

\subsection{Lessons learned from T cells: membrane targeting of SLP76}

SLP65 has a functional homologue in T cells, the cytosolic adaptor protein SLP76 (SH2 domain-containing leukocyte adaptor protein of $76 \mathrm{kDa}$ ). SLP76 has only about $30 \%$ sequence homology, but similar domain structure to SLP65 (for review, see Koretzky et al., 2006). SLP76 was first discovered as a substrate of the Syk-family PTK, ZAP-70, downstream of the T cell antigen receptor (TCR) (Bubeck Wardenburg et al., 1996). It transduces signals from the activated TCR to the intracellular signaling elements in a similar manner to SLP65 by mediating the assembly of the $\mathrm{Ca}^{2+}$ initiation complex, consisting of SLP76, PLCY1 and Itk (for review, see Koretzky et al., 2006). Likewise, the overexpression of SLP76 in the Jurkat T cell line has a positive regulatory effect on TCR signaling (Bubeck Wardenburg et al., 1996; Motto et al., 1996), while deficiency of SLP76 leads to down-regulation of PLCY1 activity and $\mathrm{Ca}^{2+}$ mobilization, resulting in defective ERK and NFAT activation (Yablonski et al., 1998).

Even though the SLP adaptors share functional and structural similarities, and have overlapping sets of interaction partners, they use different modes of translocation to their site of action. Plasma membrane recruitment of SLP76 is mediated via a transmembrane 
adaptor protein called linker for activation of T cells (LAT). LAT constitutively localizes to the glycolipid-enriched microdomains in the plasma membrane due to its transmembrane domain and palmitoylation at two cysteine residues in the N-terminus (Zhang et al., 1998b; Zhang et al., 1999). Recent studies have shown that a fraction of LAT is also found in intracytoplasmic vesicles, which traffic between their intracellular location and the cell surface (Bonello et al., 2004; Purbhoo et al., 2010). LAT is phosphorylated on several tyrosine residues by the TCR-activated kinase ZAP-70 (Zhang et al., 1998a) and upon phosphorylation recruits several SH2 domain-containing proteins, including Gads (Grb2related adaptor protein). Gads uses its $\mathrm{SH} 3$ domain to constitutively associate with a proline-rich region in SLP76 (Liu et al., 1999). Phosphorylation of LAT therefore results in the recruitment of the Gads-SLP76 complex to the TCR.

Following identification of LAT as the critical signal transducer in TCR signaling and the linker for membrane anchoring of SLP76, a similar molecule was predicted to exist in B cells. The search has identified one promising candidate, a transmembrane adaptor structurally related to LAT, which was named Linker of activated B cells (LAB) or Non-T cell activation linker (NTAL). This protein undergoes inducible tyrosine phosphorylation upon BCR engagement and interacts with several signaling molecules, including Grb2 and Sos. Moreover, NTAL can partially rescue the phenotype of LAT deficiency, when expressed in a lat ${ }^{-/}$Jurkat T cell line (Brdicka et al., 2002; Janssen et al., 2003). Based on these studies, it was proposed that NTAL could recruit SLP65 via Grb2, like LAT recruits SLP76 via Gads. However, further investigations have shown that NTAL does not represent a direct functional analog of LAT. NTAL indeed positively regulates $\mathrm{Ca}^{2+}$ mobilization in B cells, but it doesn't nucleate the formation of the $\mathrm{Ca}^{2+}$ initiation complex. Instead, LAT forms a functional complex with Grb2 and relieves the Grb2-mediated inhibition of $\mathrm{Ca}^{2+}$ mobilization. Moreover, no association of NTAL with SLP65 or PLCY2 has been reported and it was shown that NTAL mediates its regulatory function without affecting SLP65 and PLCY2 phosphorylation (Stork et al., 2004).

Presumably due to the different modes of plasma membrane recruitment, SLP65 and SLP76 cannot functionally substitute each other. SLP76 cannot rescue the phenotype of SLP65 deficiency when expressed in s/p65 $5^{-1-}$ B cells and vice versa (Ishiai et al., 2000; Abudula et al., 2007). However, co-expression of SLP76 and LAT in s/p65 ${ }^{-1-}$ DT40 cells does restore the elevation of cytosolic $\mathrm{Ca}^{2+}$ and activation of MAPKs (Wong et al., 2000). This indicates that the anchors that SLP65 uses for membrane attachment cannot be used by SLP76. In support of this hypothesis, modification of SLP76 to enable it to bind 
the SLP65-interaction partner CIN85, which contributes to SLP65 membrane translocation, renders SLP76 partially functional in s/p65 ${ }^{-/}$DT40 cells (Oellerich et al., 2011). Thus, it appears that different pathways evolved for the membrane targeting of SLP65 and SLP76, specified by the non-common interaction partners of the two proteins.

\subsection{Aims of this work}

The adaptor protein SLP65 plays an essential role in B cell activation and development, as demonstrated by the studies of SLP65-deficient mice and human-patients. SLP65 is involved in the initiation of BCR signaling, where it coordinates the assembly of the $\mathrm{Ca}^{2+}$ initiation complex at the plasma membrane. In order to carry out its function, SLP65 relocates from the cytosol to the cell surface upon BCR ligation. The mechanism of this membrane translocation is not entirely clear. The main goal of this thesis is to elucidate the molecular details of SLP65 membrane recruitment. In order to understand the membrane targeting process of SLP65 I address the following questions and issues:

1) What is the contribution of the individual domains of SLP65 for plasma membrane recruitment, and how do they cooperate for efficient targeting of SLP65? How does the molecular structure of SLP65 relate to its function? In order to investigate this aspect, a collaboration with the group of Prof. Griesinger at the Max-Planck Institute for Biophysical Chemistry is established and the secondary and tertiary structure of SLP65 is analyzed by NMR.

2) What is the molecular mechanism, by which the N-terminal region of SLP65, predicted leucine zipper, supports membrane targeting of SLP65 and what is the respective ligand? Is the $\mathrm{N}$ terminus of SLP65 required for all stages of B cell development? In order to study the function of the SLP65 $\mathrm{N}$ terminus, different point mutants of SLP65 are expressed and analyzed in SLP65-deficient B cell lines and mouse primary $B$ cells. The reported interactions of the $N$ terminus with Calmodulin and PIPs are investigated by means of biochemical assays, such as immunoprecipitation and liposome floatation.

3) What is the nature and relevance of pre-formed signaling complexes containing SLP65 and CIN85 in resting B cells? To answer this question, the subcellular distribution of SLP65 variants in resting and stimulated cells is investigated using confocal laser scanning microscopy and biochemical assays, such as subcellular fractionation. 


\section{Materials and Methods}

\subsection{Materials}

\subsubsection{Instruments}

$\underline{\text { Instrument }}$

Bio-Photometer

CD spectrometer Chirascan

Cell culture incubator HeraCell 150

Cell counter Countess ${ }^{\mathrm{TM}}$

Chemi Lux Imager

Confocal laser scanning microscope TCS SP2

DynaPro MSXTC

Electrophoresis power supply EPS 301

Electrophoresis system SDS-PAGE

Electrophoresis system Agarose gels

Incubator Kelvitron ${ }^{\circledR} \mathrm{t}$

Incubator Unitron Plus

Laminar flow hood HERA safe

Light microscope TELAVAL 31

Microplate reader PowerWave 340

NanoDrop 2000

PCR thermocycler

$\mathrm{pH}$-meter

Rocking shaker

Semi-dry transfer unit TE 77

Shaker

Sonicator

Table top centrifuge $5417 \mathrm{R}$

Thermomixer comfort
Manufacturer

Eppendorf

Applied Photophysics

Heraeus

Invitrogen

Intas systems

Leica

Wyatt technology

Amersham Biosciences

Bio-Rad

Peqlab

Heraeus

INFORS

Heraeus

Zeiss

BioTek

Thermo Scientific

Eppendorf

InoLab®

Neolab

GE Healthcare

GFL

Bandelin Sonoplus

Eppendorf

Eppendorf 
Ultracentrifuge Sorvall Discovery M150 SE

UV-illuminator

Vortex Genie 2

Water purification system Milli-Q

Waterbath

\subsubsection{Software}

Description

Corel DRAW X5

Dynamics V6

Endnote $\mathrm{X} 4$

FACS Diva

FlowJo

Image $\mathrm{J}$

Leica confocal software

Microsoft Office

OriginPro $8.5 \mathrm{G}$

pDRAW 32

ZEN 2011

\subsubsection{Consumables}

Description

Blotting Paper Whatman ${ }^{\mathrm{TM}}$

Cell culture equipment (pipettes, cryo tubes, culture dishes)

$70 \mu \mathrm{m}$ Cell strainer

Chromatography columns

Dialysis tubing SERVAPOR ${ }^{\circledR}$

4-well imaging chambers

Imaging dishes
Thermo scientific

Intas systems

Scientific industries

Milipore TM Sartorius

Schütt Labortechnik

Application

Graphic editing

DLS data processing

Bibliography editing

Flow cytometry analysis

Flow cytometry analysis

Image processing and analysis

Confocal image processing

Text and figure editing

DLS data analysis

DNA sequence analysis

Confocal image processing
Manufacturer

GE Healthcare

Greiner bio-one

BD Falcon

Bio-Rad

Serva

Lab Tek

MoBiTec 
MACS columns (LS)

Nitrocellulose membrane Hybond ECL

PCR tubes

Pipettes

Pipette tips

1.5 and $2 \mathrm{ml}$ tubes

15 and $50 \mathrm{~mL}$ tubes

\subsubsection{Chemicals and reagents}

\section{Reagent}

Acetic acid

Acrylamide/bis-acrylamide

Agarose

Ampicillin

Ammonium chloride

Ammonium persulfate (APS)

Bovine Serum Albumin (BSA) powder

BSA solution

Bromophenol blue

Calcium chloride

Chicken Serum

Chloroform

Coomassie Brilliant Blue R-250

Dimethylsulfoxide (DMSO)

DNA Ladder GeneRulerTM 1 kb

DNA 6X loading dye

Di-oleoyl phosphatidylcholine (DOPC)

Di-oleoyl phosphatidylethanolamine (DOPE)

Ethylenediamine-tetraacetic acid (EDTA)
Miltenyi Biotec

Amersham Biosciences

Sarstedt

Gilson

Greiner bio-one

Greiner bio-one

Falcon

\section{Manufacturer}

Roth

Roth

Peqlab

Roth

Roth

Roth

Serva

NEB

Merck

Merck

Sigma

Roth

Roth

Roth

Fermentas

Fermentas

Sigma

Sigma

Roth 
Ethidium Bromide

Ethanol

Fetal Calf Serum (FCS)

D-glucose

Glutathione Sepharose beads

L-glutamine

Glycine

Glycerol

HEPES

Hydrogen peroxide

Indo-1

Igepal $®$ CA-630 (NP-40)

Isopropyl- $\beta$-D-thiogalactopyranoside (IPTG)

Isopropanol

Kanamycin

Lipopolysaccharide (LPS)

Luminol

$\beta$-Mercaptoethanol

Magnesium chloride

Methanol

Deoxynucleotide solution set (dNTPs)

Nycodenz

Quinacrine

Para-hydroxycoumaric acid

Penicillin/Streptomycin

Phosphatidyl choline (PC)

Phosphatidyl ethanolamine (PE)

Phosphatidyl serine (PS)

Pluronic acid
Roth

Roth

Biochrom

Roth

GE Healthcare

Biochrom

Roth

Roth

Roth

Roth

Molecular Probes

Sigma

Roth

Roth

Roth

Sigma

Sigma

Roth

Roth

Roth

NEB

Sigma

Sigma

Sigma

Sigma

Avanti Polar Lipids

Avanti Polar Lipids

Avanti Polar Lipids

Molecular Probes 
Polybrene (Hexadimethrine bromide)

Potassium chloride

Potassium dihydrogen phosphate

Prestained Protein Marker

Protease Inhibitor Cocktail (P2714)

Protein A/G Agarose beads

Puromycin

Sephadex G-50

Sodium azide

Sodium cholate

Sodium chloride

Sodium dihydrogen phosphate

Sodium dodecyl sulphate (SDS)

Sodium fluoride

Sodium hydroxide

2x Taq Master Mix

Trans-IT

Tris base

Triton-X

Trypsin

Tween-20

X-gal

Yeast extract
Sigma

Roth

Merck

NEB

Sigma-Aldrich

Santa Cruz

InvivoGen

Sigma

Roth

Sigma

Roth

Roth

Roth

Roth

Roth

Quiagen

Mirus

Roth

Roth

Gibco

Roth

Roth

Roth

\subsubsection{Buffers and solutions}

Description

Blocking solution

Blotting buffer

\section{Composition}

$5 \%$ BSA, $0.01 \% \mathrm{NaN}_{3}$ in TBS-Tween

$39 \mathrm{mM}$ Glycine, $48 \mathrm{mM}$ Tris, $0.0375 \%$ SDS, $0.001 \% \mathrm{NaN}_{3}$, $20 \%$ methanol 
CD buffer

Coomassie staining

solution

6x DNA loading buffer

Erythrocyte lysis buffer

HP150 buffer

Krebs-Ringer bufer

$\left(+\mathrm{Ca}^{2+}\right)$

Laemmli buffer

LEW buffer

ECL solution

Solution A

Solution B

Lysis buffer with NP-40

PBS

Polybrene solution

SDS-PAGE running buffer

Separating gel

Stacking gel

TAE buffer

TBS-T
$20 \mathrm{mM} \mathrm{NaPO}_{4}, 300 \mathrm{mM} \mathrm{NaF}, \mathrm{pH} 8.0$

0.1\% Coomassie Brilliant Blue R-250, 40\% methanol, 10\% acetic acid

$10 \mathrm{mM}$ Tris/HCl, pH 7.6, 0.03\% Bromophenol blue, $60 \mathrm{mM}$ EDTA, 60\% glycerol

$144 \mathrm{mM} \mathrm{NH}_{4} \mathrm{Cl}, 17 \mathrm{mM}$ Tris/HCl, $\mathrm{pH} 7.6$

20 mM HEPES, 150 mM NaCl, pH 7.4

$140 \mathrm{mM} \mathrm{NaCl}, 4 \mathrm{mM} \mathrm{KCl}, 1 \mathrm{mM} \mathrm{MgCl}$, 10mM D-glucose, $10 \mathrm{mM}$ HEPES $\left(1 \mathrm{mM} \mathrm{CaCl}_{2}\right)$

30 mM Tris/HCl pH 6.8, 100 mM DTT, 10\% glycerol, 3\% SDS, $0.02 \%$ Bromophenol blue

$50 \mathrm{mM} \mathrm{NaH}_{2} \mathrm{PO}_{4}, 300 \mathrm{mM} \mathrm{NaCl}, \mathrm{pH} 8.0$

$4 \mathrm{ml}$ solution $\mathrm{A}, 400 \mu \mathrm{L}$ solution $\mathrm{B}, 1.2 \mu \mathrm{L} 30 \% \mathrm{H}_{2} \mathrm{O}_{2}$ $250 \mathrm{mg} / \mathrm{ml}$ Luminol in $0.1 \mathrm{M}$ Tris/HCl pH 8.6

$1.1 \mathrm{~g} / \mathrm{L}$ para-hydroxycoumaric acid in DMSO

0.5\% NP-40, 50 mM Tris pH 8, $150 \mathrm{mM} \mathrm{NaCl}, 5 \mathrm{mM} \mathrm{NaF}$, $1 \mathrm{mM} \mathrm{Na}_{3} \mathrm{VO}_{4}$, protease inhibitors

$137 \mathrm{mM} \mathrm{NaCl}, 2.4 \mathrm{mM} \mathrm{KCl}, 4.3 \mathrm{mM} \mathrm{Na}_{2} \mathrm{HPO}_{4} \times 12 \mathrm{H}_{2} \mathrm{O}$,

$1.4 \mathrm{mM} \mathrm{KH}_{2} \mathrm{PO}_{4}, \mathrm{pH} 7.4$

$3 \mathrm{mg} / \mathrm{ml}$ Polybrene in PBS

$25 \mathrm{mM}$ Tris, $192 \mathrm{mM}$ glycine, 0.1\% SDS

$375 \mathrm{mM}$ Tris/Hcl pH 8.8, 10\% acrylamide, 0.25 mM EDTA, $0.1 \%$ SDS, $0.1 \%$ TEMED, $0.1 \%$ APS

$125 \mathrm{mM}$ Tris/HCl pH 6.8, $4.8 \%$ acrylamide, $0.25 \mathrm{mM}$ EDTA, 0.1\% TEMED, $0.1 \%$ APS

$40 \mathrm{mM}$ Tris/acetic acid pH 7.8, $10 \mathrm{mM} \mathrm{NaOAc}, 1 \mathrm{mM}$

EDTA, pH 8

20 mM Tris, 137 mM NaCl, 0.1\% Tween20, pH 7.6 


\subsubsection{Media}

Description

$\mathrm{R}-10$ medium

Serum-free R-10 medium

DT40 medium

Plat E medium

Freezing medium

LB medium

LB agar

\section{Composition}

RPMI 1640 (Merck), 10\% FCS, $50 \mathrm{U} / \mathrm{ml}$ penicillin, $50 \mu \mathrm{g} / \mathrm{ml}$ streptomycin

RPMI 1640 (Merck), $50 \mathrm{U} / \mathrm{ml}$ penicillin, 50 g/ml streptomycin

RPMI 1640 (Merck), 3 mM L-glutamine, 1\% chicken serum, $50 \mathrm{U} / \mathrm{ml}$ penicillin, $50 \mu \mathrm{g} / \mathrm{ml}$ streptomycin

DMEM (Merck), 10\% FCS, $50 \mathrm{U} / \mathrm{ml}$ penicillin, $50 \mu \mathrm{g} / \mathrm{ml}$ streptomycin $10 \%$ DMSO in FCS $10 \mathrm{~g} / \mathrm{L}$ trypton, $5 \mathrm{~g} / \mathrm{L}$ yeast extract, $10 \mathrm{~g} / \mathrm{L} \mathrm{NaCl}$ $15 \mathrm{~g}$ Agar in 1L LB medium

\subsubsection{Enzymes}

Name

Phusion High-Fidelity DNA polymerase

Pfu DNA polymerase

Calf Intestinal Phosphatase (CIP)

Type II restriction endonucleases

T4 DNA ligase
Manufacturer

NEB

Promega

NEB

NEB

NEB

\subsubsection{Ready-made reaction kits}

\section{Description}

Invisorb ${ }^{\circledR}$ Spin Plasmid Mini Two

Pure Yield тм Plasmid Midiprep System

RNAeasy ${ }^{\circledR}$ Mini Kit

The Original TA cloning ${ }^{\circledR}$ Kit

Wizard ${ }^{\circledR}$ SV Gel and PCR clean up Kit

\section{Manufacturer}

Invitek

Promega

Qiagen

Invitrogen

Promega 
First strand cDNA synthesis kit

Coomassie Plus - The Better Bradford Assay ${ }^{\mathrm{TM}}$ Reagent

Pierce ${ }^{\circledR}$ BCA Protein Assay Kit

Protino ${ }^{\circledR} \mathrm{Ni}$-IDA 1000

Penta His HRP Conjugate kit

Mouse B cell isolation kit

Mini Extruder kit
Fermentas

Thermo Scientific

Thermo Scientific

Macherey Nagel

Quiagen

Milteny Biotec

Avanti Polar Lipids

\subsubsection{Oligonucleotides}

All oligonucleotides were produced by Eurofins MWG Operon by the HPSF method and supplied as a lyophilized powder, which was subsequently reconstituted with distilled water to a final concentration of $100 \mathrm{pmol} / \mu \mathrm{L}$.

$\begin{array}{lll}\text { Oligonucleotide } & \text { Sequence }\left(5^{\prime} \rightarrow 3^{\prime}\right) & \text { Application } \\ \text { Bglll_SLP65_fw } & \text { TAATAGATCTCGACAAGCTTAATAAAATAACC } & \text { cloning } \\ & \text { GTCC } & \\ \text { Notl_SLP65_rev } & \text { TAATGCGGCCGCTTATGAAACTTTAACTGCAT } & \text { cloning } \\ & \text { ACTTCAG } & \\ \text { Bglll_dNSLP65_fw } & \text { TAATAGATCTGACTACGCTTCAGAGAGCCCTG } & \text { cloning } \\ \text { Ndel_SLP65_fw } & \text { CATATGATGGACAAGCTTAATAAAATAACCG } & \text { cloning } \\ \text { hSIp_rev_stopXho } & \text { TTCTCTCGAGTTATGAAACTTTAACTGCAT } & \text { cloning } \\ \text { SLP65_L18A_fw } & \text { GAAGTTGAGGCAGCTTCAAAAGATG } & \text { mutagenesis } \\ \text { SLP65_L18A_rev } & \text { GGACCATCTTTTGAGCCTGCCTCAACT } & \text { mutagenesis } \\ \text { SLP65_M21A_fw } & \text { GAGGCAGCTTCAAAAGGCGGTCCATGATATT } & \text { mutagenesis } \\ & \text { A } & \\ \text { SLP65_M21A_rev } & \text { TTAATATCATGGACCGCCTTTTGAAGCTGCCT } & \text { mutagenesis } \\ \text { SLP65_V22A_fw } & \text { CAGCTTCAAAAGATGGCCCATGATATTAAAAA } & \text { mutagenesis } \\ & \text { C } & \\ \text { SLP65_V22A_rev } & \text { GTTTTTAATATCATGGGCCATCTTTTGAAGCT } & \text { mutagenesis }\end{array}$


SLP65_132G_fw AAACAATGAAGGTGGAGGAATGAATAAAATCA mutagenesis AA

SLP65_I32G_rev TTGATTTTATTCATTCCTCCACCTTCATTGTTT mutagenesis

SLP65_M33A_fw CAATGAAGGTGGAATAGCGAATAAAATCAAAA mutagenesis AG

SLP65_M33A_rev CTTTTTGATTTTATTCGCTATTCCACCTTCATT mutagenesis G

SLP65_I36A_fw GGAATAATGAATAAAGCCAAAAAGCTAAAAGT mutagenesis C

SLP65_I36A_rev GACTTTTAGCTTTTTGGCTTTATTCATTATTCC mutagenesis

SLP65_L39A_fw GAATAAAATCAAAAAGGCAAAAGTCAAAGCAC mutagenesis C

SLP65_L39A_rev GGTGCTTTGACTTTTGCCTTTTTGATTTTATTC mutagenesis

Bglll_Amph_fw TAATAGATCTCGCCGACATCAAGACGGGCAT cloning CT

Notl_Amph_rev TAATGCGGCCGCCTACTCCAGGCGCCGCGTG cloning AA

BgllI_Asyn_fw TAATAGATCTCGATGTGTTCATGAAAGGACTT cloning TC

Notl_Asyn_rev_tAATGCGGCCGCTTAGGCTTCAGGCTCATAG cloning TCTTG

Amph_dN_fw GGTGATCAGCATGCTGACGACTACGCTTCAG cloning AGAGCCCTG

Amph_dN_rev GCTCTCTGAAGCGTAGTCGTCAGCATGCTGA cloning TCACCGAGT

Asyn_dN_fw GAGCAGTGGTGACTGGTGACTACGCTTCAGA cloning GAGCCCTG 


\begin{tabular}{|c|c|c|}
\hline Asyn_dN_rev & GCTCTCTGAAGCGTAGTCACCAGTCACCACT & cloning \\
\hline & GCTCCTCCA & \\
\hline Bglll_chCaM_fw & AGATCTCGGATCTGCAATGGCTGATCAACTGA & cloning \\
\hline & CAGAAG & \\
\hline Notl_chCaM_rev & GCGGCCGCTCACTTCGCTGTCATCATTTG & cloning \\
\hline SH2_Nt_fw & TGCAGTTAAAGTTTCATAAATGGACAAGCTTA & cloning \\
\hline & ATAAAATAACCG & \\
\hline $\mathrm{SH} 2 \_\mathrm{Nt}$ rev & ATTTTATTAAGCTTGTCCATTTATGAAACTTTA & cloning \\
\hline & ACTGCATACTTCAGTC & \\
\hline BamHI_CIN_fw & TAATGGATCCCGTGGAGGCCATAGTGGAGTT & cloning \\
\hline & TG & \\
\hline dCC_Nt_fw & GAAGGAAAACCAAAGATGGAGCCTGACAAGC & cloning \\
\hline & TTAATAAAATAACCGTCC & \\
\hline dCC_Nt_rev & GGACGGTTATTTTATTAAGCTTGTCAGGCTCC & cloning \\
\hline & ATCTTTGGTTTTCCTTC & \\
\hline GS_part1_rev & GGAACACTTGGAGGTGCTTTGACTTTTAGCTT & cloning \\
\hline & TTTGATTTTATTCATTATTC & \\
\hline GS_part2_fw & GAATAATGAATAAAATCAAAAAGCTAAAAGTC & cloning \\
\hline & AAAGCACCTCCAAGTGTTCCTGGATCAG & \\
\hline GS_part2_rev & ССАСТTССТGАACСTGАTCCTCCGGAACCACT & cloning \\
\hline & TCCAGATCCAGAACCAC & \\
\hline GS_part3_fw & GATCTGGAAGTGGTTCCGGAGGATCAGGTTC & cloning \\
\hline & AGGAAGTGGCTCTGGAAG & \\
\hline GS_part3_rev & CAATGCCTCTTCAGCAGACTTTCGATCACAGG & cloning \\
\hline & CTCCAGCATACCATGGC & \\
\hline GS_part4_fw & TGCTGGAGCCTGTGATCGA & cloning \\
\hline & AAGTCTGCTGAAGAGGCATTG & \\
\hline GS_Syk_fw & GGTTCGGGATCCGGGAGCAATTCCGCCAGCA & cloning \\
\hline & GCGGCATGGCTG & \\
\hline
\end{tabular}




$\begin{array}{lll}\text { GS_Syk_rev } & \text { CAGCCATGCCGCTGCTGGCGGAATTGCTCCC } & \text { cloning } \\ & \text { GGATCCCGAAC } & \\ \text { Notl_Syk_(SH2)2_r } & \text { TAATGCGGCCGCCGGAAGTTGTGGACGGCCT } & \text { cloning } \\ \text { ev } & \text { C } & \\ \text { BgllI_GS_S65_fw } & \text { TAATAGATCTCGGATCAGGTTCTGGCTCAGGA } & \text { cloning } \\ & \text { AGCG } & \\ \text { Agel_GS_S65_rv } & \text { TAATACCGGTTTTGAAACTTTAACTGCATACTT } & \text { cloning } \\ & \text { CAGTCT } & \\ \text { Notl_Cit_rev } & \text { TAATGCGGCCGCTTTACTTGTACAGCTCGTC } & \text { cloning } \\ \text { pMSCVfw } & \text { CCCTTGAACCTCCTCGTTCGACC } & \text { sequencing } \\ \text { pMSCVrev } & \text { CAGACGTGCTACTTCCATTTGTC } & \text { sequencing } \\ \text { EGFP_C_fw } & \text { GTCCTGCTGGAGTTCGTG } & \text { sequencing } \\ \text { EGFP_C_rev } & \text { AGCTGCAATAAACAAGTT } & \text { sequencing } \\ \text { T7 } & \text { TAATACGACTCACTATAGG } & \text { sequencing }\end{array}$

\subsubsection{Vectors and Constructs}

\subsubsection{Vectors}

\begin{tabular}{|c|c|c|}
\hline$\underline{\text { Vector }}$ & Application & Source \\
\hline pCRII & T/A cloning & Invitrogen \\
\hline pHCMV-VSV-G & $\begin{array}{l}\text { expression of } v s v-g \text { cDNA for the production of } \\
\text { recombinant retroviruses }\end{array}$ & Dr. M. Engelke \\
\hline pMSCVpuro & $\begin{array}{l}\text { expression of retroviraly transduced cDNA in } \\
\text { eukaryotic cells }\end{array}$ & Clontech \\
\hline pMSCVpuro Cit & $\begin{array}{l}\text { expression of retrovirally transduced cDNA with } \\
\text { an N-terminal Citrine tag in eukaryotic cells }\end{array}$ & Dr. M. Engelke \\
\hline pET16b TEV & $\begin{array}{l}\text { expression of cDNA with an } \mathrm{N} \text {-terminal } \mathrm{His}_{6} \text { tag } \\
\text { in bacteria }\end{array}$ & Dr. S. Becker \\
\hline pGEX-4T1 & expression of cDNA with a GST-tag in bacteria & GE Healthcare \\
\hline
\end{tabular}




\subsubsection{Constructs}

The constructs generated in this thesis were cloned using standard procedures described in the Methods section. For the generation of eukaryotic expression vectors carrying the cDNA of interest with an N-terminal Citrine tag, the cDNA was amplified by PCR, digested with $\mathrm{Bgll} / \mathrm{BamHI}$ and Notl restriction enzymes and ligated into the pMSCVpuro Cit vector digested with BgllI and Notl. For the generation of prokaryotic expression vectors carrying the cDNA of interest with an N-terminal $\mathrm{His}_{6}$ tag, the cDNA was amplified by PCR, digested with Ndel and Xhol and ligated into the pET16bTEV vector digested with Ndel and Xhol. The mouse Amph and Asyn cDNA was first cloned into the pCRII vector and then sub-cloned into pMSCVpuro Cit.

\begin{tabular}{|c|c|c|}
\hline$\underline{\text { Construct }}$ & Description & Source \\
\hline pCRII hSLP65 & Human SLP65 & Dr. V. Bremes \\
\hline $\begin{array}{l}\text { pMSCVpuro Cit } \\
\text { hSLP65WT }\end{array}$ & N-terminal Citrine-tag, human SLP65 & Dr. M. Engelke \\
\hline $\begin{array}{l}\text { pMSCVpuro Cit } \\
\text { hSLP } 65 \Delta \mathrm{N}\end{array}$ & N-terminal Citrine-tag, human SLP65 $\Delta$ aa 1-50 & Dr. M. Engelke \\
\hline $\begin{array}{l}\text { pMSCVpuro } \\
\text { Citrine }\end{array}$ & N-terminal Citrine-tag & Dr. M. Engelke \\
\hline $\begin{array}{l}\text { pMSCVpuro Cit } \\
\text { chCaM }\end{array}$ & N-terminal Citrine-tag, chicken Calmodulin & This thesis \\
\hline $\begin{array}{l}\text { pMSCVpuro Cit } \\
\text { hSLP65I25A }\end{array}$ & N-terminal Citrine-tag, human SLP65 I25A & Dr. N. Hermann \\
\hline $\begin{array}{l}\text { pMSCVpuro Cit } \\
\text { hSLP65I25K }\end{array}$ & N-terminal Citrine-tag, human SLP65 I25K & Dr. N. Hermann \\
\hline $\begin{array}{l}\text { pMSCVpuro Cit } \\
\text { hSLP65L18K }\end{array}$ & N-terminal Citrine-tag, human SLP65 L18K & Dr. M. Engelke \\
\hline $\begin{array}{l}\text { pMSCVpuro Cit } \\
\text { hSLP65L18A }\end{array}$ & N-terminal Citrine-tag, human SLP65 L18A & This thesis \\
\hline $\begin{array}{l}\text { pET16bTEV } \\
\text { hSLP65WT }\end{array}$ & N-terminal $\mathrm{His}_{6}$-tag, human SLP65 & Dr. N. Hermann \\
\hline
\end{tabular}




\begin{tabular}{|c|c|c|}
\hline $\begin{array}{l}\text { pET15b } \\
\text { hSLP65 } \Delta N\end{array}$ & $\mathrm{~N}$-terminal $\mathrm{His}_{6}$-tag, human SLP65 $\Delta$ aa 1-48 & Dr. N. Hermann \\
\hline $\begin{array}{l}\text { pET16bTEV } \\
\text { hSLP65L18K }\end{array}$ & N-terminal $\mathrm{His}_{6}$-tag, human SLP65 L18K & This thesis \\
\hline $\begin{array}{l}\text { pET16bTEV } \\
\text { hSLP65L18A }\end{array}$ & 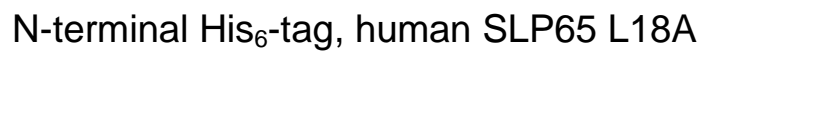 & This thesis \\
\hline $\begin{array}{l}\text { pET16bTEV } \\
\text { hSLP65I32G }\end{array}$ & N-terminal $\mathrm{His}_{6}$-tag, human SLP65 I32G & This thesis \\
\hline $\begin{array}{l}\text { pET16bTEV } \\
\text { hSLP65M33A }\end{array}$ & N-terminal $\mathrm{His}_{6}$-tag, human SLP65 M33A & This thesis \\
\hline $\begin{array}{l}\text { pET16bTEV } \\
\text { hSLP65I36A }\end{array}$ & N-terminal His ${ }_{6}$-tag, human SLP65 I36A & This thesis \\
\hline pCRII mAmph & Mouse Amphyphisin & This thesis \\
\hline pCRII mAsyn & Mouse a-Synuclein & This thesis \\
\hline $\begin{array}{l}\text { pMSCVpuro Cit } \\
\text { N-BAR- } \triangle \text { NSLP65 }\end{array}$ & $\begin{array}{l}\text { N-terminal Citrine-tag, mouse Amphiphysin aa } \\
1-240 \text { fused to human SLP65 } \Delta \text { aa } 1-50\end{array}$ & This thesis \\
\hline $\begin{array}{l}\text { pMSCVpuro Cit } \\
\text { ALPS- } \triangle \text { NSLP65 }\end{array}$ & $\begin{array}{l}\text { N-terminal Citrine-tag, mouse } \alpha \text {-Synuclein aa } 1- \\
74 \text { fused to human SLP65 } \Delta \text { aa } 1-50\end{array}$ & This thesis \\
\hline $\begin{array}{l}\text { pMSCVpuro Cit } \\
\text { N-BAR- } \triangle \text { NSLP65 } \\
\text { M23 }\end{array}$ & $\begin{array}{l}\text { N-terminal Citrine-tag, BAR domain of mouse } \\
\text { Amphiphysin fused to human SLP65 } \Delta \text { aa 1-50, } \\
\text { R248.313A }\end{array}$ & This thesis \\
\hline $\begin{array}{l}\text { pMSCVpuro Cit } \\
\text { hSLP65 } \Delta \text { N-Nt }\end{array}$ & $\begin{array}{l}\text { N-terminal Citrine-tag, human SLP65 } \Delta \text { aa } 1-50 \\
\text { fused to human SLP65 aa 1-50 at the C- } \\
\text { terminus }\end{array}$ & This thesis \\
\hline $\begin{array}{l}\text { pMSCVpuro Cit } \\
\text { hCIN85 } \triangle \text { CC-Nt }\end{array}$ & $\begin{array}{l}\text { N-terminal Citrine-tag, human CIN85 1-599 } \\
\text { fused to human SLP65 aa 1-50 }\end{array}$ & This thesis \\
\hline $\begin{array}{l}\text { pMSCVpuro Cit } \\
\text { hSLP65M21A }\end{array}$ & N-terminal Citrine-tag, human SLP65 M21A & This thesis \\
\hline
\end{tabular}




\begin{tabular}{|c|c|c|}
\hline $\begin{array}{l}\text { pMSCVpuro Cit } \\
\text { hSLP65M21G }\end{array}$ & N-terminal Citrine-tag, human SLP65 M21G & This thesis \\
\hline $\begin{array}{l}\text { pMSCVpuro Cit } \\
\text { hSLP65V22A }\end{array}$ & N-terminal Citrine-tag, human SLP65 V22A & This thesis \\
\hline $\begin{array}{l}\text { pMSCVpuro Cit } \\
\text { hSLP65I32G }\end{array}$ & N-terminal Citrine-tag, human SLP65 I32G & This thesis \\
\hline $\begin{array}{l}\text { pMSCVpuro Cit } \\
\text { hSLP65M33A }\end{array}$ & N-terminal Citrine-tag, human SLP65 M33A & This thesis \\
\hline $\begin{array}{l}\text { pMSCVpuro Cit } \\
\text { hSLP65I36A }\end{array}$ & N-terminal Citrine-tag, human SLP65 I36A & This thesis \\
\hline $\begin{array}{l}\text { pMSCVpuro Cit } \\
\text { hSLP65L39G }\end{array}$ & N-terminal Citrine-tag, human SLP65 L39G & This thesis \\
\hline $\begin{array}{l}\text { pMSCVpuro Cit } \\
\text { hSLP65GS }\end{array}$ & $\begin{array}{l}\text { N-terminal Citrine-tag, human SLP } 65 \text { with a GS } \\
\text { linker between aa } 50-330 \text { (see Figure } 4.2 \text { for } \\
\text { complete sequence) }\end{array}$ & This thesis \\
\hline $\begin{array}{l}\text { pMSCVpuro Cit } \\
\text { CC- } \triangle \text { NSLP65 GS }\end{array}$ & $\begin{array}{l}\text { Human CIN85 aa 594-665 fused to human } \\
\text { SLP65 } \Delta \text { aa 1-50 with a GS linker between aa } \\
\text { 50-330, C-terminal Citrine-tag }\end{array}$ & This thesis \\
\hline $\begin{array}{l}\text { pMSCVpuro Cit } \\
\text { SLP65GS }\end{array}$ & $\begin{array}{l}\text { N-terminal Citrine-tag, human SLP65 aa 1-50, } \\
\text { GS linker, human Syk aa 2-277 (tSH2) }\end{array}$ & This thesis \\
\hline $\begin{array}{l}\text { pMSCVpuro Cit } \\
\text { GS tSyk(SH2)2 }\end{array}$ & $\begin{array}{l}\text { N-terminal Citrine-tag, GS linker, human Syk } \\
\text { tSH2 }\end{array}$ & This thesis \\
\hline $\begin{array}{l}\text { pMSCVpuro Cit } \\
\text { hSLP65Y96F- }\end{array}$ & $\begin{array}{l}\text { N-terminal Citrine-tag, human SLP65 Y96Fnew } \\
\text { Btk site (see Figure } 4.4 \text { for domain structure) }\end{array}$ & This thesis \\
\hline $\begin{array}{l}\text { pGEX-4T1 Itk } \\
\text { SH2 }\end{array}$ & N-terminal GST tag, human Btk $\mathrm{SH} 2$ domain & $\begin{array}{l}\text { Dr. Niklas } \\
\text { Engels }\end{array}$ \\
\hline
\end{tabular}




\subsubsection{Antibodies}

\subsection{Primary antibodies}

The antibodies were used for Western blot analysis (WB), Immunoprecipitation (IP) or BCR-stimulation (S). For Western blotting, primary antibodies were used at the dilution recommended by the supplier in TBS-T with 1\% (w/v) BSA and $0.01 \%(\mathrm{v} / \mathrm{v}) \mathrm{NaN}_{3}$. The antibody concentrations used for BCR-stimulation and immunoprecipitation are mentioned in the respective methods sections.

\begin{tabular}{|c|c|c|}
\hline Antibody & Supplier & Application \\
\hline$\alpha$-human BLNK, mouse monoclonal $\lg _{2 a}$ & BD Biosciences & WB \\
\hline a-human BLNK (\#3587), rabbit polyclonal & CST & WB \\
\hline$\alpha$-GFP, mouse monoclonal $\lg _{1} \mathrm{~K}$ & Roche & IP, WB \\
\hline a-chicken IgM (M4) & Biozol & $\mathrm{S}$ \\
\hline a-Akt1, rabbit monoclonal & CST & WB \\
\hline$\alpha-H s v 2$, rabbit polyclonal & Dr. K. Kühnel & WB \\
\hline a-human pBLNK (Y96), rabbit polyclonal & CST & WB \\
\hline $\mathrm{F}_{\mathrm{ab}}$ fragment goat anti-human IgM & Jackson & $\mathrm{S}$ \\
\hline$F_{a b}$ fragment goat anti-mouse $\lg M+\lg G$ & Jackson & $\mathrm{S}$ \\
\hline$\alpha-p Y(4 G 10)$, monoclonal mouse $\lg _{2 b}$ & Merck Millipore & WB \\
\hline a-human PLCY2 (Q20), rabbit polyclonal & Santa Cruz & WB \\
\hline
\end{tabular}

\subsubsection{Secondary antibodies}

All secondary antibodies were used for Western blotting at a 1: 10000 dilution in TBS-T.

Antibody

Goat a-rabbit IgG, HRPO-conjugated

Goat a-mouse IgG, HRPO-conjugated
Supplier

Southern biotech

Southern biotech 


\subsubsection{Biological material}

\subsubsection{Bacterial strains}

\section{$\underline{\text { Strain }}$}

One Shot ${ }^{\circledR}$ TOP10F' chemo-competent E.coli

One Shot ${ }^{\circledR} \mathrm{BL}-21$ (DE3) chemo-competent E.coli
Supplier

Life Technologies

Life Technologies

\subsubsection{Eukaryotic cell lines}

\section{DT40 (ATCC ® CRL-2111}

The DT40 cell line is chicken B cell line, derived from an avian leucosis virus-induced bursal lymphoma (Baba \& Humphries, 1984). It expresses IgM on the cell surface. DT40 cells have a high ratio of targeted to random DNA integration, which allows for easy gene modification via homologous recombination (Winding \& Berchtold, 2001).

\section{DG75 (DSMZ-No: ACC 83)}

The DG75 cell line was derived from a pleural effusion of a 10-year old child with Burkitt's lymphoma. It expresses IgM coupled to the $\mathrm{k}$ light chain on the cell surface (Ben-Bassat et al., 1977).

\subsection{Methods}

\subsubsection{Molecular biology methods}

\subsubsection{Polymerase Chain Reaction (PCR)}

PCR was used for the amplification of DNA fragments from CDNA or plasmid DNA, which were subsequently used for further cloning procedures. This method allows specific DNA amplification in vitro with a thermostable DNA polymerase; the method was originally described by (Saiki et al., 1988). PCR was set up in a total volume of $25 \mu \mathrm{L}$ with $200 \mu \mathrm{M}$ dNTPs (NEB), $0.2 \mu \mathrm{M}$ of each primer, $1 \mathrm{X}$ GC buffer (NEB), $0.4 \cup$ Phusion High-Fidelity DNA polymerase (NEB) and 10-50 ng template DNA. The parameters for the cycler program are summarized below. The amplification times and annealing temperatures varied according to the primers and the templates used. Higher annealing temperature was used for GC-rich templates and primers with higher melting temperature. 
Table 3.1 Cycler parameters for a standard PCR program.

\begin{tabular}{|c|c|c|c|c|}
\hline$\underline{\text { Step }}$ & Explanation & Temperature $\left({ }^{\circ} \mathrm{C}\right)$ & $\underline{\text { Time }}$ & Number of cycles \\
\hline 1 & Initial denaturation & 94 & $2 \min$ & 1 \\
\hline 2 & Denaturation & 94 & $30 \mathrm{sec}$ & \multirow[t]{3}{*}{$30-35$} \\
\hline 3 & Annealing & $55-65$ & $30 \mathrm{sec}$ & \\
\hline 4 & Elongation & 68 & $30 \mathrm{sec}$ to $2 \mathrm{~min}$ & \\
\hline 5 & Final elongation & 68 & $10 \mathrm{~min}$ & 1 \\
\hline 6 & Pause & 14 & $\infty$ & 1 \\
\hline
\end{tabular}

\subsubsection{Overlap extension PCR}

Overlap extension PCR was used for the fusion of two DNA fragments with overlapping ends. First, the two fragments were amplified with the conventional PCR using primers, designed to contain the overlapping ends. Subsequently, an overlap extension PCR was set up with $200 \mu \mathrm{M}$ dNTPs (NEB), 1X GC buffer (NEB), $0.2 \mu \mathrm{L}$ Phusion High-Fidelity DNA polymerase (NEB) and 20-100 ng of each template DNA. The following program was used for overlap extension PCRs:

Table 3.2 Cycler parameters for the overlap extension PCR, part 1.

\begin{tabular}{|c|c|c|c|c|}
\hline$\underline{\text { Step }}$ & Explanation & Temperature $\left({ }^{\circ} \mathrm{C}\right)$ & $\underline{\text { Time }}$ & Number of cycles \\
\hline 1 & Initial denaturation & 94 & $2 \min$ & 1 \\
\hline 2 & Denaturation & 94 & $30 \mathrm{sec}$ & \multirow[t]{3}{*}{15} \\
\hline 3 & Annealing & $55-58$ & $30 \mathrm{sec}$ & \\
\hline 4 & Elongation & 68 & $30 \mathrm{sec}$ to $2 \mathrm{~min}$ & \\
\hline 5 & Final elongation & 68 & $10 \mathrm{~min}$ & 1 \\
\hline 6 & Pause & 14 & $\infty$ & 1 \\
\hline
\end{tabular}

Following initial amplification of the extended template, forward and reverse primers for the final amplification product were added to a final concentration of $0.2 \mu \mathrm{M}$ and the amplification was continued for 25-30 cycles with increasing annealing temperature to ensure specificity. 
Table 3.3 Cycler parameters for the overlap extension PCR, part 2.

\begin{tabular}{|l|l|l|l|l|}
\hline$\underline{\text { Step }}$ & Explanation & $\underline{\text { Temperature }\left({ }^{\circ} \mathrm{C}\right)}$ & Time & Number of cycles \\
\hline 1 & Initial denaturation & 94 & $2 \mathrm{~min}$ & 1 \\
\hline 2 & Denaturation & 94 & $30 \mathrm{sec}$ & $25-30$ \\
\hline 3 & Annealing & $58+0.2^{\circ} \mathrm{C} /$ cycle & $30 \mathrm{sec}$ & \\
\hline 4 & Elongation & 68 & $30 \mathrm{sec}$ to $2 \mathrm{~min}$ & \\
\hline 5 & Final elongation & 68 & $10 \mathrm{~min}$ & 1 \\
\hline 6 & Pause & 14 & $\infty$ & 1 \\
\hline
\end{tabular}

An overlap extension PCR was used to generate the cDNA encoding chimeric fusion proteins N-BAR- $\triangle$ NSLP65, ALPS- $\Delta$ NSLP65, SLP65 GS, CC- $\triangle$ NSLP65GS and SLP65 GS-Sykt(SH2)2.

\subsubsection{Site directed mutagenesis}

Site directed mutagenesis was used to introduce single point mutations into the $\mathrm{N}$-terminal region of SLP65. Overlapping forward and reverse primers carrying the desired mutation were used to amplify the plasmid containing the wild type s/p65 gene (pCRII SLP65) by conventional PCR. Pfu polymerase was used and the elongation time was set to 4 min. Following amplification, the PCR product was purified and treated with $1 \mu \mathrm{L}$ Dpnl endonuclease (NEB) for $30 \mathrm{~min}$ at $37{ }^{\circ} \mathrm{C}$ in order to digest the non-mutated plasmid, which was used as the PCR template.

\subsubsection{Restriction enzyme digestion}

PCR fragments and plasmids were digested with restriction endonucleases for further cloning applications. The digests were set up in a total volume of $25 \mu \mathrm{L}$ with $2.5 \mu \mathrm{L}$ appropriate 10X buffer (NEB), $2.5 \mu \mathrm{L} 1 \mathrm{mg} / \mathrm{mL}$ BSA (NEB), 0.3-1 $\mu \mathrm{L}$ restriction enzyme and 0.2-1 $\mu \mathrm{g}$ DNA. Digests were performed at $37{ }^{\circ} \mathrm{C}$ for 1 to 12 hours. For the digests of plasmid DNA with only one type of enzyme subsequent treatment with $1 \mu \mathrm{L}$ of CIP was performed for $30 \mathrm{~min}$ at $37^{\circ} \mathrm{C}$ in order to dephosphorylate the $5^{\prime}$ ends and prevent plasmid re-ligation.

\subsubsection{Agarose gel electrophoresis}

Agarose gel electrophoresis was used to separate PCR products or DNA fragments resulting from restriction digests. $1 \%$ agarose gels ( $2 \%$ for the separation of fragments 
smaller than $500 \mathrm{bp}$ ) were prepared in 1 X TAE buffer with $0.5 \mu \mathrm{g} / \mathrm{mL}$ etidium bromide. The DNA fragments to be separated were pre-mixed with the 6X DNA loading dye (Fermentas) and loaded on the gel together with the $1 \mathrm{~kb}$ DNA ladder (Fermentas) for size determination. Electrophoresis was performed at $200 \mathrm{~mA}$ and $100 \mathrm{~V}$ for 20-50 min (longer for larger DNA fragments). An UV illuminator (Intas Systems) was used to visualize the DNA.

\subsubsection{Purification and gel extraction of DNA fragments}

PCR products and DNA fragments resulting from digestion with restriction enzymes were purified prior to further cloning procedures. The Wizard ${ }^{\circledR} \mathrm{SV}$ Gel and PCR clean up Kit (Promega) was used according to the manufacturer's protocol. PCR products were either purified directly following PCR or separated by size on an agarose gel first. In the latter case, DNA bands of the appropriate size were excised from the gel with a scalpel and DNA was extracted from the gel according to the instructions. The DNA fragments were eluted in 30-100 $\mu \mathrm{L}$ Nuclease-free water (Promega).

\subsubsection{Ligation of DNA fragments}

For the ligation of DNA fragments, ligation mixture with a total volume of $10 \mu \mathrm{L}$, containing $1 \mathrm{X}$ ligation buffer (NEB), $\sim 20 \mathrm{ng}$ digested vector DNA, $\sim 50 \mathrm{ng}$ digested insert DNA and 1 $\mu \mathrm{L}$ T4 DNA ligase (NEB) was set up. The ligation mixture was incubated at $16{ }^{\circ} \mathrm{C}$ for 4 to 12 hours.

\subsubsection{Cloning of PCR fragments via T/A cloning}

For cloning of PCR fragments into the PCRII vector, purified PCR fragments were incubated with the $2 x$ Taq master mix for 1 hour at $72{ }^{\circ} \mathrm{C}$ in order to add " $A$ " overhangs to the DNA ends. Following this treatment, PCR fragments were purified again and ligated into the PCRII vector according to the standard procedures described above.

\subsubsection{Transformation of chemo-competent E.coli}

E. coli Top10F- or BL21 competent cells (kindly prepared by Ines Heine) were thawed on ice. Top10F- cells were used for the amplification of plasmids while BL21 cells were used for protein expression. The ligation mix or 100-200 ng plasmid DNA was added onto the cells and incubated on ice for $20 \mathrm{~min}$. Following a heat shock treatment at $42{ }^{\circ} \mathrm{C}$ for 45 sec to $1 \mathrm{~min}$, the cells were briefly incubated on ice and $200 \mu \mathrm{L}$ antibiotic-free LB medium was added. The cells were subsequently incubated at $37{ }^{\circ} \mathrm{C}$ for $30 \mathrm{~min}$ and plated on 
ampicillin (100 $\mu \mathrm{g} / \mathrm{ml})$ - or kanamycin $(50 \mu \mathrm{g} / \mathrm{ml})$-containing LB plates. The plates were incubated at $37^{\circ} \mathrm{C}$ for $12-16$ hours.

\subsubsection{Isolation of plasmid DNA}

Single colonies were picked from bacterial plates, resuspended in LB medium supplemented with ampicillin or kanamycin and grown overnight at $37{ }^{\circ} \mathrm{C}$. Plasmid DNA was isolated from $2-4 \mathrm{~mL}$ or $100-200 \mathrm{~mL}$ of overnight bacterial culture using either the Invisorb ${ }^{\circledR}$ Spin Plasmid Mini Two kit or the Pure Yield тM Plasmid Midiprep kit, respectively, according to the manufacturer's instructions. The concentration of purified plasmid DNA was determined with the NanoDrop spectrophotometer (Thermo Scientific). 1-1.2 $\mu$ g plasmid DNA was sent for sequencing with an appropriate primer to Microsynth AG, Goettingen.

\subsubsection{Total RNA isolation}

Total RNA was isolated from mouse brain tissue with the RNeasy ${ }^{\circledR}$ Mini kit (Quiagen), according to the protocol provided with the kit. RNA was eluted in $100 \mu \mathrm{L}$ RNase-free water.

\subsubsection{2 cDNA synthesis}

The RNA isolated from the mouse brain tissue was used as a template for cDNA synthesis. The First strand cDNA synthesis kit (Fermentas) was used to produce cDNA by reverse transcription. The obtained CDNA was used as a template for PCR in order to amplify the fragments encoding Amphiphysin and a-Synuclein lipid-binding domains.

\subsubsection{Biochemical methods}

\subsubsection{Expression and purification of recombinant GST proteins}

E.coli BL21 cells, transformed with the GST-fusion constructs (pGEXT backbone), were grown overnight and diluted 1:100 in 50-200 mL LB medium supplemented with ampicillin. The cultures were grown at $37^{\circ} \mathrm{C}$ to $\mathrm{OD}_{600} 0.6$ and protein expression was induced with $0.1 \mathrm{mM}$ IPTG. After 4 hours bacteria were harvested by centrifugation at $6000 \mathrm{rpm}$ at $4{ }^{\circ} \mathrm{C}$ for $20 \mathrm{~min}$ and the pellets were resuspended in 1-4 mL PBS. For lysis, the bacteria were sonicated 3 times for 30 sec $(5 \times 10 \%$ cycles, 50\% power). Following sonication, Triton-X was added to a final concentration of $0.01 \%$ and bacterial lysates were incubated on ice for $30 \mathrm{~min}$. Subsequently, the lysates were centrifuged at $6000 \mathrm{rpm}$ at $4{ }^{\circ} \mathrm{C}$, and the 
supernatant was used for incubation with Glutathione Sepharose beads (GE Healthcare) overnight, while rotating at $4{ }^{\circ} \mathrm{C}$. The beads were washed 3 times with PBS and resuspended in an appropriate amount of PBS in order to get a 50-60 \% (v/v) slurry suspension. To quantify the amount of bound GST-fusion proteins, 5 and $10 \mu \mathrm{L}$ of slurry was mixed with $2 X$ Laemmli buffer and loaded on an SDS-PAGE gel together with known amounts of BSA (NEB) and pre-stained protein marker (NEB). Following protein separation the gel was stained with Coomasie staining solution for 5-10 min while shaking and de-stained with tap water overnight.

\subsubsection{Expression and purification of recombinant His-tagged proteins}

For the expression of $\mathrm{N}$-terminal $\mathrm{His}_{6}$-tagged SLP65 protein, pET16b plasmids were transformed into E.coli BL21. Bacterial cultures were grown as described above for the GST-fusion proteins. Protein expression was induced with $1 \mathrm{mM}$ IPTG. After 4 hours, bacteria were harvested, resuspended in LEW buffer and lysed as described above. $\mathrm{His}_{6}{ }^{-}$ tagged proteins were purified with the Protino Ni-IDA 1000 kit (Macherey Nagel) according to the manufacturer's instructions. The proteins were eluted from the column in $1 \mathrm{~mL} 250$ $\mathrm{mM}$ imidazole-containing LEW buffer. The proteins were dialyzed overnight against LEW buffer without imidazole and stored with protease inhibitors (Sigma) and $0.01 \% \mathrm{NaN}_{3}$ at 4 ${ }^{\circ} \mathrm{C}$ for maximum one week.

\subsubsection{Photometric determination of protein concentration}

For the determination of protein concentration, BCA or Bradford methods was used. Several dilutions of the protein of interest were prepared in the appropriate buffer. A series of BSA dilutions with concentrations of 0.125 to $1.5 \mathrm{mg} / \mathrm{mL}$ BSA (NEB) were prepared in the same buffer. The standards and protein samples were pipetted into a 96-well plate and an appropriate amount of Bradford (Thermo scientific) or BCA (Thermo scientific) reagent was added. The absorbance was measured with the microplate reader PowerWave 340 (BioTek) at $595 \mathrm{~nm}$ for the Bradford assay or at $562 \mathrm{~nm}$ for the BCA assay. A standard curve was plotted and used for calculation of protein concentrations.

\subsubsection{Preparation of cleared cellular lysates (CCLs)}

Cleared cellular lysates were prepared from resting and BCR-stimulated DT40 cells and used to analyze the protein expression level by Western blotting, or further used for protein immunoprecipitation or affinity purification experiments. The cells were harvested and washed once with PBS. 1-5 x 10 cells were resuspended in serum free R10 medium 
and "starved" for $30 \mathrm{~min}$ at $37{ }^{\circ} \mathrm{C}$, in order to reduce the basal level of protein phosphorylation. Following starvation, the cells were left unstimulated or were stimulated for different amounts of time with $2 \mu \mathrm{g} / \mathrm{mL}$ a-chicken IgM (M4) antibody. Stimulation was done at $37^{\circ} \mathrm{C}$ and stopped by transferring the cells on ice. Subsequently, the cells were harvested at $10000 \mathrm{rpm}$ at $4{ }^{\circ} \mathrm{C}$ for $1 \mathrm{~min}$, resuspended in $1 \mathrm{~mL}$ NP-40 lysis buffer and incubated for $45-60 \mathrm{~min}$ at $4{ }^{\circ} \mathrm{C}$, while rotating. Following lysis, the lysates were centrifuged at $14000 \mathrm{rpm}$ at $4{ }^{\circ} \mathrm{C}$ for $20 \mathrm{~min}$ and the supernatant was transferred into a new Eppendorf tube. The lysates were then mixed with Laemmli buffer, incubated for 5 min at $95^{\circ} \mathrm{C}$ and loaded on a gel for analysis by immunoblotting.

\subsubsection{Affinity purification (AP)}

For affinity purification, $2.5-5 \times 10^{7}$ cells per sample were used to prepare CCLs as described above. Following centrifugation, $900 \mu \mathrm{L}$ of the CCLs were incubated with 20-30 $\mu \mathrm{L}$ of Glutathione Sepharose beads (GE Healthcare) (50\% slurry suspension) bound to GST-fusion proteins for 4 hours, rotating at $4{ }^{\circ} \mathrm{C}$. The beads were collected by centrifugation at $300 \mathrm{~g}$, at $4{ }^{\circ} \mathrm{C}$ for $4 \mathrm{~min}$ and washed 3 times with NP-40 lysis buffer. Finally, the supernatant was removed and the beads were resuspended in $50 \mu \mathrm{L} 2 \mathrm{X}$ Laemmli buffer. The samples were incubated at $95^{\circ} \mathrm{C}$ for $5 \mathrm{~min}$ to elute the proteins and loaded on a gel for analysis by immunoblotting.

\subsubsection{Protein immunoprecipitation (IP)}

2.5-5 $\times 10^{7}$ cells per sample were used to prepare CCLs, as described above. After centrifugation, $900 \mu \mathrm{L}$ of the lysates were incubated with $1 \mu \mathrm{g}$ of precipitating antibody ( $\alpha$ GFP) overnight, while rotating at $4{ }^{\circ} \mathrm{C}$. On the next day $20 \mu \mathrm{L}$ of Protein A/G Agarose beads (Santa Cruz) (25\% suspension) was added and the samples were incubated for 45 min rotating at $4{ }^{\circ} \mathrm{C}$. The beads were collected by centrifugation at $300 \mathrm{~g}$, at $4{ }^{\circ} \mathrm{C}$ for $4 \mathrm{~min}$ and washed 3 times with NP-40 lysis buffer. The supernatant was removed and the beads were resuspended in $50 \mu \mathrm{L} 2 \mathrm{X}$ Laemmli buffer. The samples were incubated at $95^{\circ} \mathrm{C}$ for 5 min to elute the protein from the beads and loaded on a gel for analysis by immunoblotting.

\subsubsection{SDS polyacrylamide gel electrophoresis (SDS-PAGE)}

SDS-PAGE was used to separate proteins under denaturing conditions by size, as originally described by Laemmli (Laemmli, 1970). The proteins were first concentrated in a stacking gel and then separated in a resolving gel (discontinuous SDS gel system). The 
$10 \%$ resolving gel was prepared with 10\% acrylamide-bis-acrylamide (17.5:1), $375 \mathrm{mM}$ Tris $\mathrm{pH} 8.8,0.1 \%$ SDS, $0.001 \%$ APS and $0.001 \%$ TEMED. The 5\% stacking gel was prepared with 5\% acrylamide-bis-acrylamide (17.5:1), $125 \mathrm{mM}$ Tris pH 6.8, 0.001\% APS and $0.001 \%$ TEMED. Electrophoresis was performed in SDS-PAGE running buffer at a constant current of $15 \mathrm{~mA}$ for the stacking gel, and $30 \mathrm{~mA}$ for the separating gel. Prestained protein marker (NEB) was loaded on the gel together with the samples for molecular weight determination. Following electrophoresis, the gel was subjected to Western blot analysis or stained with Coomasie staining solution for direct visualization of proteins.

\subsubsection{Western blot (WB) analysis}

Following the separation by SDS-PAGE, the proteins from the gel were transferred to a nitrocellulose membrane. A semi-dry blotting method was used. Nitrocellulose membrane and two pieces of Whatman paper were cut according to the size of the gel and soaked in Blotting buffer. The transfer apparatus was assembled as follows: anode, Whatman paper, nitrocellulose membrane, gel, Whatman paper, cathode. The proteins were transferred at $200 \mathrm{~mA}$ and $16 \mathrm{~V}$ for 1 hour. Following transfer, the blot was incubated in the blocking solution at RT for 1 hour. Afterwards, the blot was incubated with the primary antibody solution at $4{ }^{\circ} \mathrm{C}$ overnight. The next day the blot was washed 3 times for $15 \mathrm{~min}$ with TBS-T at RT. Afterwards, the blot was incubated with the secondary antibody solution at RT for 1 hour. Finally, the blot was washed 3 times with TBS-T and developed with Luminol solution using digital imaging system (Chemi Lux Imager Intas) for specific detection of proteins.

\subsubsection{Preparation of SUVs by detergent exclusion}

The preparation of liposomes with different methods and the liposome floatation assays were performed at the lab of Dr. K. Kühnel, using resources of the department of Neurobiology, Max-Planck Institute for Biophysical Chemistry. Phosphatidylcholine (PC), phosphatidylethanolamine (PE), phosphatidylserine (PS) (all Avanti Polar Lipids) and Texas-Red labeled PE (Life Technologies) were reconstituted with chloroform to a final concentration of $20 \mathrm{mg} / \mathrm{ml}$. Appropriate amounts of lipids were pipetted to a $2 \mathrm{~mL}$ Eppendorf tube with round bottom to a total lipid amount of $1 \mathrm{mg}$. Lipid films were dried at RT for 2 hours and either directly used for liposome preparation, or stored at $-20^{\circ} \mathrm{C}$ for 4 6 weeks. The lipid films were rehydrated with $150 \mu \mathrm{L} 3 \%$ sodium cholate solution in LEW buffer. Sephadex G-50 was resuspended in LEW buffer at a concentration of $0.05 \mathrm{~g} / \mathrm{mL}$ 
and this solution was used to pack the chromatography columns (Bio-Rad). Lipiddetergent mixture was carefully loaded on packed columns and overlaid with $5 \mathrm{~mL}$ LEW buffer. The pink fraction containing SUVs was collected $(400-500 \mu \mathrm{L})$ into an Eppendorf tube.

\subsubsection{Preparation of LUVs by extrusion}

Lipid films were prepared as described above and rehydrated with $1 \mathrm{~mL} L E W$ buffer. The liposome suspension was extruded through $400 \mathrm{~nm}$ and $100 \mathrm{~nm}$ filters with the mini extruder kit (Avanti Polar Lipids) using $1 \mathrm{~mL}$ Hamilton syringes.

\subsubsection{Liposome floatation}

$5 \mu \mathrm{L}$ of the recombinant $\mathrm{His}_{6}$-tagged SLP65 solution, with a protein concentration of 1-5 $\mu \mathrm{M}$ was mixed with $45 \mu \mathrm{L}$ SUV or LUV solution and incubated at RT for $15 \mathrm{~min}$. For the comparison of protein binding to SUVS and LUVS, the liposome concentration was adjusted prior to incubation with the protein. For this purpose, the absorbance of TexasRed at $592 \mathrm{~nm}$ in SUV and LUV samples was measured with the NanoDrop spectrophotometer (Thermo scientific) and the more concentrated suspension was diluted with an appropriate amount of LEW buffer. The lipid-protein mixture was mixed with $50 \mu \mathrm{L}$ 80\% Nycodenz solution (in LEW buffer) and transferred to ultracentrifugation polycarbonate tubes (Beckman). $50 \mu \mathrm{L}$ 30\% Nycodenz solution was carefully added on top. Finally, the gradient was overlaid with $30 \mu \mathrm{L}$ LEW buffer. Ultracentrifugation was performed at $55000 \mathrm{rpm}$ at $4{ }^{\circ} \mathrm{C}$ for $90 \mathrm{~min}$. Following ultracentrifugation, six fractions, 30 $\mu \mathrm{L}$ each, were taken for analysis of protein distribution by immunoblotting, mixed with Laemmli buffer and incubated at $95^{\circ} \mathrm{C}$ for $5 \mathrm{~min}$ to denature the proteins.

\subsubsection{Preparation of SUVs for NMR and CD spectroscopy}

For NMR and CD spectroscopy, 75\% (w/v) DOPC, 25\% (w/v) DOPE SUVs were used. The lipids for SUV preparation were kindly provided by C. Schwiegk (department of NMRbased biology, Max-Planck Institute for Biophysical Chemistry). Lipid films were prepared as described above and rehydrated with $1 \mathrm{~mL}$ HP150 buffer for a final total lipid concentration of $12 \mathrm{mM}$. The lipid suspension was sonicated for $45 \mathrm{sec} 20$ times (5x10\% cycles, $60 \%$ power) on ice. Afterwards, the sonicated lipid suspension was extruded through $100 \mathrm{~nm}$ and $50 \mathrm{~nm}$ filters. 


\subsubsection{Cell biology methods}

\subsubsection{Culturing of eukaryotic cells}

All cells were passaged under the laminar flow hood (Heraeus) and cultured at $37^{\circ} \mathrm{C}$ in a humidified atmosphere with $5 \% \mathrm{CO}_{2}$. Adherent cells (PlatE) were cultured in DMEM medium, supplemented with $10 \% \mathrm{FCS}, 50 \mathrm{U} / \mathrm{mL}$ penicillin and $50 \mu \mathrm{g} / \mathrm{mL}$ streptomycin. PlatE cells were treated with $0.05 \mathrm{M}$ Trypsin/EDTA and incubated for $1 \mathrm{~min}$ at $37^{\circ} \mathrm{C}$ prior to passaging. Every two weeks PlatE cells were selected with $10 \mu \mathrm{g} / \mathrm{mL}$ blasticidin and 2.5 $\mu \mathrm{g} / \mathrm{mL}$ puromycin. Suspension cells (all B cell lines and primary B cells) were cultured in RPMI medium, supplemented with $10 \%$ FCS, $50 \mathrm{U} / \mathrm{mL}$ penicillin and $50 \mu \mathrm{g} / \mathrm{mL}$ streptomycin (R10). For culturing of DT40 cells, R10 medium was also supplemented with $1 \%$ chicken serum and $3 \mathrm{mM}$ L-glutamine.

\subsubsection{Harvesting and counting of cells}

All cells were harvested by centrifugation in a table top centrifuge at RT, $1200 \mathrm{rpm}$ for 4 min unless indicated otherwise. The automated cell counter Countess ${ }^{\mathrm{TM}}$ (Invitrogen) was used to count the cells.

\subsubsection{Freezing and thawing of cells}

Cells were thawed rapidly in a water bath at $37{ }^{\circ} \mathrm{C}$ and resuspended in $10 \mathrm{~mL}$ R10 or PlatE medium. The cells were then harvested by centrifugation, resuspended in fresh medium and transferred to the cell culture dishes.

For freezing, 0.5-1 × $10^{7}$ cells were harvested, resuspended in 1-1.4 mL freezing medium, aliquoted to 2 cryo-vials and transferred on ice. The cells were frozen at $-140{ }^{\circ} \mathrm{C}$.

\subsubsection{Isolation of primary splenic mouse B cells}

Primary B cells were isolated from the spleens of two 8-weeks old s/p65 ${ }^{-/}$mice and were used for retroviral transfection with SLP65 constructs. The spleens were prepared, homogenized with a plunger top of a syringe in a Petri dish filled with $10 \mathrm{~mL} \mathrm{R} 10$, and passed through a $70 \mu \mathrm{m}$ nylon cell strainer (BD Falcon). The cells were transferred to a 15 $\mathrm{mL}$ Falcon tube and centrifuged at $1200 \mathrm{rpm}$ at $4{ }^{\circ} \mathrm{C}$ for $6 \mathrm{~min}$. The supernatant was discarded and the cell pellet was resuspended in $2 \mathrm{~mL}$ erythrocyte lysis buffer and incubated at $4{ }^{\circ} \mathrm{C}$ for $4 \mathrm{~min}$. The reaction was stopped by the addition of $0.7 \mathrm{~mL}$ FCS. The cells were centrifuged at $1200 \mathrm{rpm}$ at $4{ }^{\circ} \mathrm{C}$ for $6 \mathrm{~min}$ and washed once with PBS. Subsequently, the cells were counted and subjected to Magnetic Activated Cell Sorting 
(MACS) using the Mouse B cell isolation kit (Miltenyi Biotec). LS MACS separation columns were used. Following isolation, the cells were counted again and resuspended in R10 medium, supplemented with $20 \mu \mathrm{g} / \mathrm{ml}$ LPS, at a cell density of $1 \times 10^{6}$ cells $/ \mathrm{mL}$.

\subsubsection{Transfection of PlatE cells by lipofection}

PlatE cells were used for the generation of infectious, replication-dependent recombinant retroviruses. The cells were co-transfected with the retroviral expression vector pMSCV containing the cDNA of interest and the pHCMV eukaryotic expression vector encoding the envelope glycoprotein from the vesicular stomatitis virus (VSV-G), to allow for production of amphotrophic viruses. One day prior to transfection PlatE cells were seeded on $6-\mathrm{cm}$ cell culture dishes, to achieve a confluence of $50-70 \%$ at the time of transfection. The transfection mix, consisting of $400 \mu \mathrm{L}$ serum-free $\mathrm{R} 10$ medium, $7 \mu \mathrm{L}$ Trans-IT transfection reagent (Mirus), $0.5 \mu \mathrm{g}$ pHCMV-VSV-G plasmid DNA and 2-3 $\mu \mathrm{g}$ pMSCV plasmid DNA, was prepared and incubated at RT for 15-45 min. The DMEM medium was removed from the PlatE cells and $4 \mathrm{~mL}$ of fresh R10 medium was added. The transfection mix was carefully pipetted onto the cells and the cells were incubated at $37^{\circ} \mathrm{C}$ for 48 hours.

\subsubsection{Retroviral transduction of DT40 and DG75 cells}

DT40 and DG75 cells were infected with recombinant retroviruses to achieve a stable expression of the transgene of interest. Viral supernatant was collected from transfected PlatE cells and centrifuged at $1200 \mathrm{rpm}$ for $4 \mathrm{~min}$ to separate the viral particles from the cells. $1 \times 10^{6}$ cells were harvested and resuspended in 3.5-4 $\mathrm{mL}$ of viral supernatant. The cells were transferred onto $6-\mathrm{cm}$ dishes and $1 \mathrm{ml}$ of fresh R10 medium, supplemented with $3 \mu \mathrm{g} / \mathrm{mL}$ Polybrene, was added. The cells were incubated with the viral supernatant at $37^{\circ} \mathrm{C}$ for 24 hours. On the next day, the viral supernatant was removed; the cells were resuspended in fresh R10 medium, and left to recover from infection at $37^{\circ} \mathrm{C}$ for 24 hours. On the following day, the cells were transferred to the selection medium (R10 or DT40 medium supplemented with $1 \mu \mathrm{g} / \mathrm{mL}$ Puromycin). The cells were kept on selection for 2-3 days and subsequently transferred to normal R10 medium.

\subsubsection{Retroviral transduction of primary mouse B cells}

For the infection of $1-5 \times 10^{6}$ LPS-stimulated splenic mouse B cells, concentrated viral supernatant from five 6-cm PlatE-seeded dishes, previously transfected with plasmid of interest by lipofection, was used. Viral supernatant was collected in $2 \mathrm{~mL}$ Eppendorf tubes 
and centrifuged in a table top centrifuge at $20000 \mathrm{~g}, 4{ }^{\circ} \mathrm{C}$ for $90 \mathrm{~min}$. Following centrifugation, $1.25 \mathrm{~mL}$ of the supernatant was discarded and the remaining $0.75 \mathrm{~mL}$ was used to resuspend the viral pellet. LPS-stimulated mouse B cells were harvested in a 50 $\mathrm{mL}$ Falcon tube and resuspended in the concentrated viral supernatant. LPS and Polybrene were added to the final concentrations of $20 \mu \mathrm{g} / \mathrm{ml}$ and $3 \mu \mathrm{g} / \mathrm{ml}$, respectively. The cells were subsequently centrifuged with the viral supernatant at $1500 \mathrm{~g}$ at $33^{\circ} \mathrm{C}$ for 3.5 hours. The supernatant was discarded and the cells were resuspended in pre-warmed R10 medium supplemented with $20 \mu \mathrm{g} / \mathrm{ml}$ LPS, at a density of $1 \times 10^{6}$ cells $/ \mathrm{mL}$.

\subsubsection{Analysis of $\mathrm{Ca}^{2+}$ flux by flow cytometry}

In order to monitor $\mathrm{BCR}$-induced $\mathrm{Ca}^{2+}$ mobilization by flow cytometry, the $\mathrm{Ca}^{2+}$ sensitive dye, polycyclic chelator Indo-1, was used. In its free, $\mathrm{Ca}^{2+}$-unbound form, Indo-1 emits fluorescence at $475 \mathrm{~nm}$; while in the $\mathrm{Ca}^{2+}$-bound form its emission shifts to $400 \mathrm{~nm}$ (Grynkiewicz et al., 1985). The ratio of Indo-1 fluorescence at $405 \mathrm{~nm}$ to that at $530 \mathrm{~nm}$ was used to monitor the changes in intracellular $\mathrm{Ca}^{2+}$ concentration. $1.5 \times 10^{6}$ cells were harvested, resuspended in $700 \mu \mathrm{L}$ RPMI medium with 5\% FCS, and transferred to darkbrown Eppendorf tubes. Indo-1 and pluronic acid were added onto the cells to the final concentrations of $1 \mu \mathrm{M}$ and $0.015 \%(\mathrm{v} / \mathrm{v})$, respectively. The cells were vortexed briefly and incubated at $30{ }^{\circ} \mathrm{C}$ for 25 min while shaking. Subsequently, $700 \mu \mathrm{L}$ R10 medium was added and the cells were incubated at $37^{\circ} \mathrm{C}$ for $10 \mathrm{~min}$. The cells were washed twice with Krebs-Ringer buffer supplemented with $1 \mu \mathrm{M} \mathrm{CaCl}{ }_{2}$. The cells were subsequently resuspended in $800 \mu \mathrm{L} \mathrm{Ca}{ }^{2+}$-containing Krebs-Ringer buffer, and incubated at RT for 10 min prior to the measurement. $\mathrm{Ca}^{2+}$ flux measurements were done with the BD LSRII flow cytometer. The ratio of fluorescence at 530 and $405 \mathrm{~nm}$ was monitored for $4 \mathrm{~min}$. $30 \mathrm{sec}$ after the start of recording, the stimulating antibody was added. DT40 cells were stimulated with $2 \mu \mathrm{g} / \mathrm{ml} \alpha$-chicken IgM (M4), DG75 cells were stimulated with $20 \mu \mathrm{g} / \mathrm{ml} \mathrm{\alpha -}$ human $\operatorname{lgM} F_{a b}$ fragment and mouse primary B cells were stimulated with $20 \mu \mathrm{g} / \mathrm{ml} \mathrm{\alpha}$ mouse IgM+lgG $F_{a b}$ fragments. The $\mathrm{Ca}^{2+}$ mobilization profiles were analyzed with the FlowJo software.

\subsubsection{Confocal laser scanning microscopy (CLSM)}

CLSM was used to analyze the subcellular localization and plasma membrane-recruitment of Citrine-tagged SLP65 variants. $5 \times 10^{5}$ cells were harvested, washed 2 times with Krebs-Ringer buffer and resuspended in $400 \mu \mathrm{L} \mathrm{Ca}{ }^{2+}$-containing Krebs-Ringer buffer. The cells were transferred to 4-well imaging chambers or polylysine-coated imaging dishes 
(used for primary mouse B cells) and allowed to settle down. The cells were imaged with the Leica TCS SP2 confocal laser scanning microscope in the department of Medical Microbiology (microscope kindly provided by Prof. Dr. Carsten Lüder). Citrine was excited at $514 \mathrm{~nm}$ and the emission was recorded at $530-600 \mathrm{~nm}$. The cells were stimulated with $2 \mu \mathrm{g} / \mathrm{ml}$ a-chicken IgM M4 (DT40 cells), $20 \mu \mathrm{g} / \mathrm{ml}$ a-human IgM Fab fragment (DG75 cells) or $20 \mu \mathrm{g} / \mathrm{ml} \alpha$-mouse IgM+lgG Fab fragment (mouse primary B cells). Images were processed and analyzed with the ImageJ software.

\subsubsection{Colocalization analysis of fluorescence signals}

For the colocalization analysis of SLP65 with quinacrine, $5 \times 10^{5}$ cells were harvested, washed once with Krebs-Ringer solution and stained with $1 \mu \mathrm{M}$ quinacrine for $30 \mathrm{~min}$ at $37^{\circ} \mathrm{C}$. Following staining, the cells were washed once with $\mathrm{Ca}^{2+}$-containing Krebs-Ringer buffer and transferred to imaging chambers. Cerulean (quinacrine) was excited at $458 \mathrm{~nm}$ and the emission was recorded at $470-500 \mathrm{~nm}$. In order to avoid spectral overlap, Citrine and Cerulean signals were scanned sequentially. Z-stacks of 8 images were taken for each fluorophore. Background substraction (Rolling-ball radius 10.0 pixels), Gauss filtering and colocalization analysis were applied with the ImageJ software.

\subsubsection{Fluorescence recovery after photobleaching (FRAP) analysis}

For FRAP analysis, $5 \times 10^{5}$ cells were harvested, washed twice with Krebs-Ringer buffer and plated on imaging dishes. The cells were imaged with a Zeiss LSM 510 confocal laser scanning microscope in the Institute of Neuro- and Sensory Physiology (microscope kindly provided by Dr. M. Niebert). Time laps series of 25 images were taken. 5 pre-bleach images were taken, then a region of interest (ROI) was chosen and photo-bleached using maximum laser power, and subsequently 20 post-bleach images were acquired to monitor fluorescence recovery. Quantification of fluorescence in the ROI of individual images at 25 time points was performed using the ImageJ software. Background subtraction and correction for fluorescence loss due to photobleaching was done.

\subsubsection{Biophysical methods}

\subsubsection{Dynamic light scattering (DLS)}

DLS was used to monitor the size distribution of liposomes. The liposomes were prepared by extrusion and detergent exclusion methods as described above, and were diluted 1:20 with LEW buffer prior to DLS analysis. DLS was performed with a DynaPro-MSXTC 
instrument (Wyatt technology) in the department of Neurobiology, Max-Planck Institute for Biophysical Chemistry. 10 measurements per sample with an acquisition time of $10 \mathrm{sec}$ were performed at $25{ }^{\circ} \mathrm{C}$. The laser power was adjusted to keep the intensity between 100000 and 2000000 counts. The results were processed with the DYNAMICS V6 software (Wyatt technology) and analyzed with the OriginPro 8.5G software.

\subsubsection{Circular dichroism spectroscopy}

Circular dichroism was used to analyze the secondary structure of SLP655-50 in the absence and presence of SUVs. The lyophilized sample of SLP65 $5_{5-50}$ was prepared and kindly provided by C. Schwiegk (department of NMR-based Structural Biology, MaxPlanck Institute for Biophysical Chemistry). The protein was reconstituted in CD buffer to a final concentration of $50 \mu \mathrm{M}$. SUVs, prepared by the method of sonication and extrusion, were used at a total lipid concentration of $0.5-2 \mathrm{mM}$. The SUVs were incubated with the protein in a CD cuvette for $15 \mathrm{~min}$ at RT prior to the measurement. Chirascan Circular Dichroism Spectrometer (Applied Photophysics) and Hellma quartz cuvettes (pathlength $0.1 \mathrm{~cm}$ ) at the department of Neurobiology, Max Planck Institute for Biophysical Chemistry were used. The CD spectrum was recorded at 190 to $260 \mathrm{~nm}$, with a step size of $1 \mathrm{~nm}$, a bandwidth of $0.5 \mathrm{~nm}$ and an averaging time of $3 \mathrm{sec}$ at RT. For baseline correction, the $C D$ spectrum of the buffer was subtracted from the CD spectrum of the protein, and $C D$ spectrum of SUVs was subtracted from the spectrum of the protein + SUVs. CD data was analyzed with the manufacturer's ProView software and the DichroWeb online CD data analysis platform (Whitmore \& Wallace, 2004; Whitmore \& Wallace, 2008). 


\section{Results}

\subsection{NMR analysis of the secondary structure of SLP65 and validation of the structural investigations in vivo}

\subsubsection{SLP65 possesses large intrinsically disordered regions}

The elucidation of the secondary and tertiary structure of a protein can help to understand many aspects of its function. We investigated the secondary structure of SLP65 by NMR in collaboration with the research group of Prof. Christian Griesinger at the department of NMR-based structural biology, Max-Planck Institute for Biophysical Chemistry. Recombinant ${ }^{15} \mathrm{~N}-{ }^{1} \mathrm{H}$ labelled SLP65 was expressed in and purified from E.coli. In addition to full length SLP65, we used a shortened construct comprising the residues 40 to 330 of human SLP65. In this construct, the parts corresponding to the N-terminal positively charged region and to the $\mathrm{SH} 2$ domain were removed in order to improve the solubility of the protein and to facilitate the residue assignment. HSQC (heteronuclear single quantum coherence) spectra of both constructs at $298 \mathrm{~K}$ were recorded. Examination of the spectra revealed that SLP65 was largely intrinsically disordered as evident from the low peak dispersion (Figure 4.1).

This result was in concert with the SLP65 structure prediction by the secondary structure prediction tools (prediction by the I-TASSER server; Zhang, 2008). The spectra of fulllength SLP65 and SLP65 ${ }_{40-330}$ could be overlaid, indicating that removing the $\mathrm{N}$ terminus and the $\mathrm{SH} 2$ domain did not interfere with the overall structure of SLP65. Moreover, the IDP (intrinsically disordered protein) character of SLP65 did not change upon in vitro phosphorylation with Syk kinase or upon binding to the $3 \mathrm{SH} 3$ domains of CIN85 (data not shown). 
${ }^{15} \mathrm{~N}-{ }^{1} \mathrm{H}$ HSQC spectrum of SLP65 and SLP65 $5_{40-330}$ at $298 \mathrm{~K}$

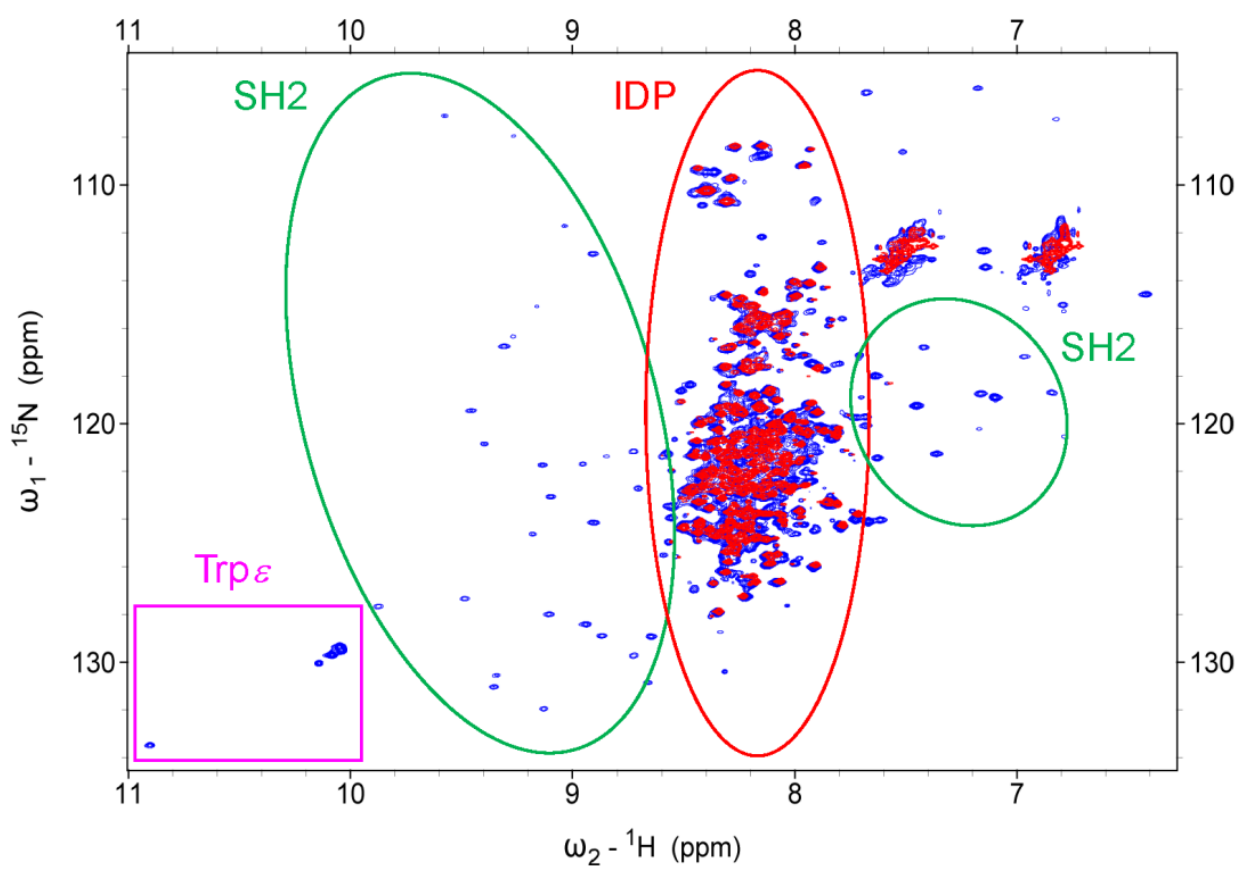

Figure 4.1 SLP65 is an IDP except for the SH2 domain.

${ }^{15} \mathrm{~N}-{ }^{1} \mathrm{H}$ HSQC spectra of full-length human SLP65 (blue) and SLP65 ${ }_{40-330}$ (red) are shown. Low peak dispersion indicates intrinsically disordered conformation. The proteins were cloned and purified by C. Schwiegk. The spectra were acquired and analysed by Dr. L. Russo.

\subsubsection{Intrinsic disorder in SLP65 is sufficient for the assembly of the $\mathrm{Ca}^{2+}$ initiation complex, but not for membrane targeting of SLP65}

The central part of SLP65 contains critical signalling elements - the inducibly phosphorylated tyrosine residues and the proline-rich regions. The NMR analysis showed that this part of SLP65 is disordered in solution. I wondered how the IDP character of SLP65 supports its function in vivo. I hypothesized that one could replace the IDP part of SLP65 by another disordered sequence and preserve the signalling function of the protein. To test this hypothesis, I generated a protein construct where the residues 50 to 330 of human SLP65 were replaced by a flexible GS (Glycine Serine)-linker. The tyrosine residues that bind Btk and PLCY2 were preserved in this construct, including a few amino acids upstream and downstream to allow for the recognition by the Syk kinase and the Btk and PLCY2 SH2 domains. This synthetic protein construct was named "SLP65 GS", and its full amino acid sequence is shown in Figure 4.2. It was expressed in SLP65-deficient DT40 cells and assessed for its ability to reconstitute $\mathrm{Ca}^{2+}$ signaling. 


\begin{tabular}{|c|c|c|c|c|}
\hline 10 & 20 & 30 & 40 & 56 \\
\hline DKLNKITVP & ASQKLRQLQK & MVHDIKNNEG & GIMNKIKKLK & VKAPPSVPGG \\
\hline 60 & 70 & 80 & 90 & 100 \\
\hline GSGSGSGSG & ¿SGSGSGSG & GSGSG & AEEN & PPPV \\
\hline 110 & 120 & 130 & 140 & 150 \\
\hline QETRPVHPA & LPFARGEYID & RSGSGSGSG & GSGSG & SGSGSGGSGS \\
\hline 160 & 170 & 180 & 190 & 200 \\
\hline SGSGS & SGSGL & VVPV & YIHP & GSG \\
\hline 210 & 220 & 230 & 240 & 250 \\
\hline SGSGSGGSS & SGSGGSGSG & SGGSGSGSGS & GGSGS & GSSGGSGSGS \\
\hline 260 & 270 & 280 & 290 & 300 \\
\hline SSGSGSNSTI & SEQEAGVLCK & PWYAGACDRS & AEEALHRSNK & DGSFLIRKSS \\
\hline 310 & 320 & 330 & 340 & 350 \\
\hline PYT & RVYN & VRFIEATK & QYALGRKKNG & EEYFGSVAE \\
\hline & 370 & & & \\
\hline
\end{tabular}

Figure 4.2 Amino acid sequence of SLP65 GS.

The residues in blue correspond to the $\mathrm{N}$-terminal membrane targeting region and to the $\mathrm{SH} 2$ domain. The tyrosine residues binding the $\mathrm{SH} 2$ domains of Btk and PLCY2 are shown in red. The cDNA encoding for this synthetic protein was synthesized in parts by MWG operons, amplified via overlap extension PCR and cloned into the pMSCVpuro vector with an $\mathrm{N}$-terminal Citrine-tag.

Confocal laser scanning microscopy (CLSM)-imaging analysis revealed that Citrinetagged SLP65 GS was localized entirely in the cytosol of resting and BCR-stimulated cells. As it was not recruited to the plasma membrane, it is unsurprising that it failed to initiate $\mathrm{Ca}^{2+}$ mobilization upon BCR stimulation (Figure $4.3 \mathrm{~B}$ and C). Since SLP65 GS lacked the CIN85-binding sites (proline rich regions), and the interaction of SLP65 and CIN85 is critical for SLP65 membrane translocation (Oellerich et al., 2011), the signaling insufficiency of SLP65 GS could be attributed to the lack of CIN85 binding. To test for this possibility, I equipped the synthetic GS construct with the CIN85 predicted coiled-coil (CC) domain. The CIN85 CC domain has been previously shown in our lab to target SLP65 to the plasma membrane upon BCR stimulation, independently of the proline-rich regions (unpublished data by J. Kühn). The CC-SLP65 M23 or CC-SLP65 GS chimeric proteins were expressed in s/p65 $5^{-1}$ DT40 cells. Whereas the CIN85 CC domain provided signaling capability to the otherwise unfunctional $\triangle N$ SLP65 M23, it failed to do so when fused to the GS construct (Figure 4.3 B). This result suggested that the mere absence of CIN85 binding is not sufficient to account for the severe $\mathrm{Ca}^{2+}$ phenotype of the synthetic SLP65 GS construct. It therefore remains unclear why SLP65 GS and CC- $\Delta N$ GS were unable to translocate to the plasma membrane upon BCR ligation, even though all known domains required for membrane targeting of SLP65 were present. This data implies that the central disordered part of SLP65 contains a previously unrecognized membrane targeting site. It 
might mediate the membrane anchoring of SLP65 via an interaction with an unknown protein ligand or contribute in a structural manner. It could also support the function of the already known membrane targeting domains, such as the $\mathrm{SH} 2$ domain or the $\mathrm{N}$ terminus of SLP65.

A)

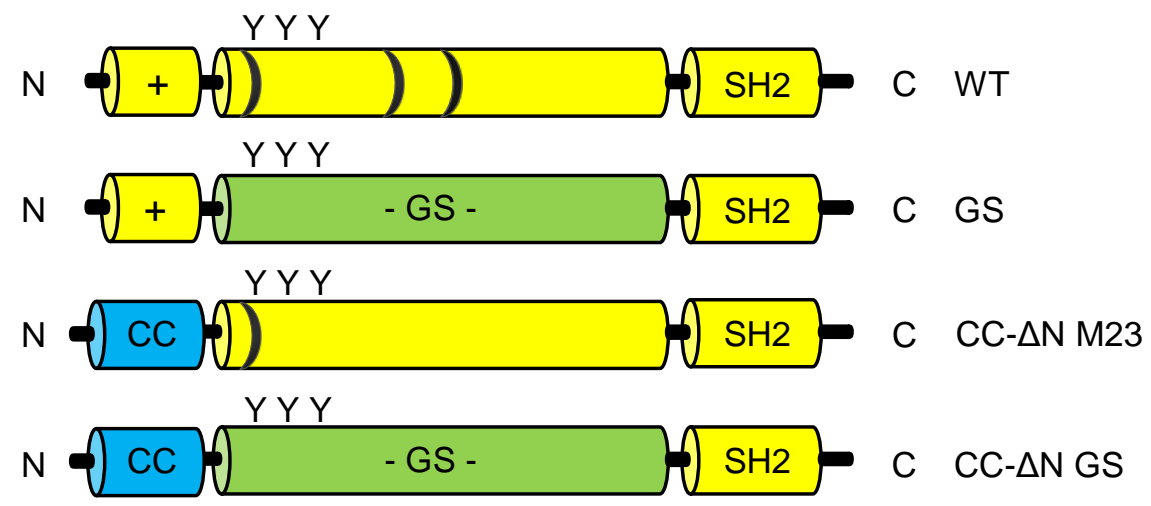

B)

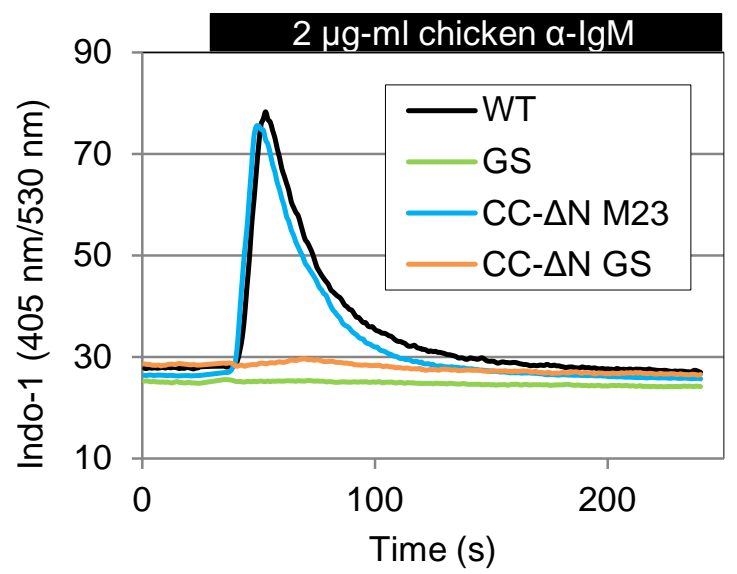

C) DT40 s/p65\%

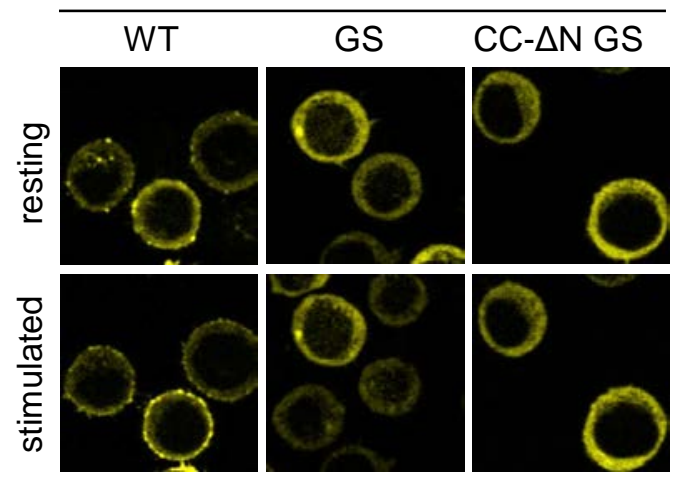

Figure 4.3 The central part of SLP65 is indispensable for membrane targeting.

A) Schematic representation of the domain organization of SLP65 WT, GS, CC- $\triangle N$ M23 and CC$\triangle N$ GS. B) The constructs represented in (A) with an N-terminal Citrine tag (not shown) were retrovirally transduced into s/p65 $5^{--}$DT40 cells. $\mathrm{Ca}^{2+}$ mobilization was measured by flow cytometry. The $\mathrm{Ca}^{2+}$ flux profiles of cells with equal Citrine expression are shown. C) Subcellular localization and plasma membrane recruitment of Citrine-tagged SLP65 WT, GS and CC- $\Delta \mathrm{N}$ was analysed by CLSM. Stimulation was performed with $2 \mu \mathrm{g} / \mathrm{ml}$ a-chicken IgM antibody. 
Due to these numerous possibilities, it is not surprising that the very complex and delicate process of membrane targeting of SLP65 was inhibited by the introduction of the disordered GS sequence. However, the data presented until now did not exclude the possibility that the intrinsic disorder in SLP65 is useful later for the assembly of the $\mathrm{Ca}^{2+}$ initiation complex; which is a distinct process downstream of plasma membrane recruitment of SLP65. To test whether this was the case, I fused a known BCR-targeting signal, the tandem SH2 domains of Syk kinase, at the C-terminus of SLP65 GS and thereby "bypassed" the natural membrane targeting step (Figure $4.4 \mathrm{~A}$ ). The tandem SH2 domains of Syk bind to phosphorylated tyrosine residues at the ITAMs of Iga and $\lg \beta$ with high affinity (Kurosaki et al., 1995; Ottinger et al., 1998). Covalently joining these domains to SLP65 has been shown to bring it to the BCR upon stimulation (Bremes, 2012).

As shown in Figure 4.4 C, SLP65 GS t(SH2)2 efficiently translocated to the plasma membrane upon $\mathrm{BCR}$ ligation. Moreover, this chimeric protein could initiate $\mathrm{Ca}^{2+}$ signaling (Figure $4.4 \mathrm{~B}$ ). This indicated that even though the GS-linker could not replace the original SLP65 sequence for membrane targeting, it was sufficient for the assembly of the $\mathrm{Ca}^{2+}$ initiation complex and $\mathrm{Ca}^{2+}$ mobilization after SLP65 was brought to the BCR. Finally, by omitting the N-terminal region of SLP65, I created a minimal protein construct that could fulfill the function of SLP65 for $\mathrm{Ca}^{2+}$ signaling. It consisted of a flexible GS-rich scaffold, including the important tyrosine residues, and a BCR-targeting signal, the tandem $\mathrm{SH} 2$ domains of Syk. This chimeric protein, termed $\Delta \mathrm{N}$ SLP65 GS t(SH2)2 could support $\mathrm{Ca}^{2+}$ mobilization upon BCR-stimulation (Figure 4.4 B). Moreover, immunoblot analysis with phospho-specific antibodies revealed that $\triangle \mathrm{N}$ SLP65 GS t(SH2)2 was efficiently tyrosinephosphorylated. It remained phosphorylated even 5 minutes after IgM stimulation, in comparison to SLP65 WT, which reached its maximum level of phosphorylation 2 minutes after BCR stimulation and showed reduced phosphorylation 5 minutes after activation (Figure 4.4 D, lanes 1-6). This was consistent with the stable association of $\Delta N$ SLP65 GS $\mathrm{t}(\mathrm{SH} 2) 2$ with the plasma membrane 5 min after BCR stimulation (data not shown). Lysates from cells expressing the SLP65 variant $Y_{\text {all }} F$, where 5 tyrosine residues known to be phosphorylated by Syk were exchanged against phenylalanine (kindly provided by Dr. M. Engelke), were used as a negative control. In this case, no phosphorylation of SLP65 upon BCR ligation was observed (Figure 4.4 D, lanes 7-9). 
A)

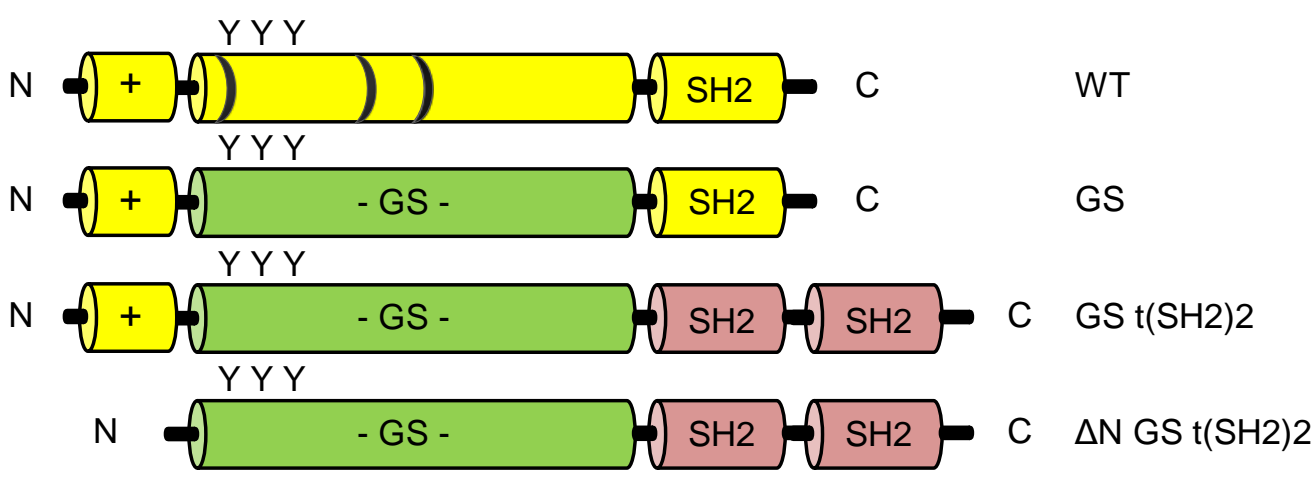

B)

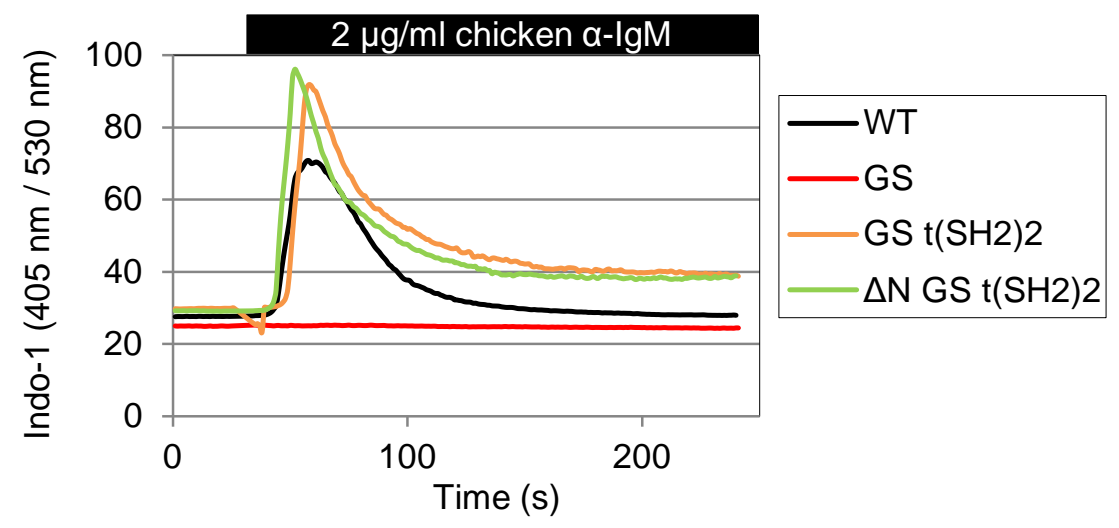

C)

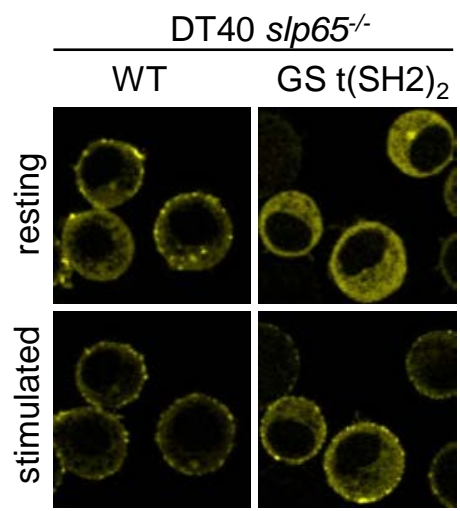

D)

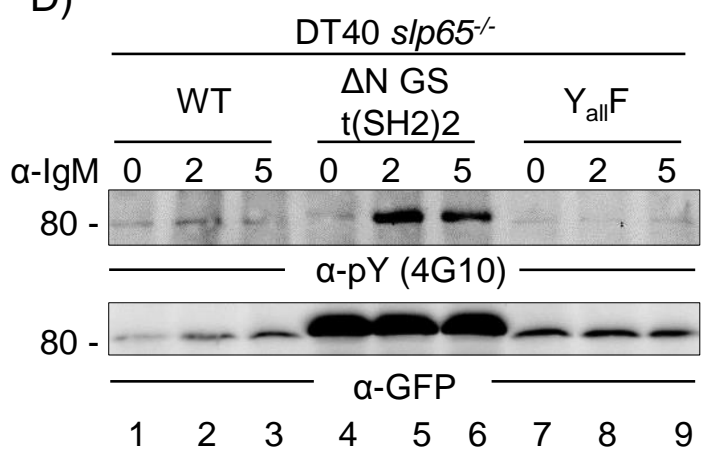

Figure 4.4 The GS linker in the central part of SLP65 is sufficient for $\mathrm{Ca}^{2+}$ signaling once SLP65 is brought to the BCR.

A) Schematic representation of the domain structure of SLP65 WT, GS, GS t(SH2)2 and $\Delta N$ GS $\mathrm{t}(\mathrm{SH} 2) 2$. B) The constructs represented in (A) with an N-terminal Citrine tag (not shown) were retrovirally transduced into slp65 $5^{--}$DT40 cells and $\mathrm{Ca}^{2+}$ mobilization was measured by flow cytometry. The $\mathrm{Ca}^{2+}$ flux profiles of cells with equal Citrine expression are shown. C) Plasma membrane recruitment of SLP65 WT or GS $\mathrm{t}(\mathrm{SH} 2) 2$ upon BCR-stimulation was analyzed by CLSM. Images were taken before and $3 \mathrm{~min}$ after stimulation with $2 \mu \mathrm{g} / \mathrm{ml} \alpha$-chicken IgM antibody. D) CCLs were prepared from resting and stimulated DT40 cells expressing either SLP65 WT, $\triangle N$ GS $\mathrm{t}(\mathrm{SH} 2) 2$ or $\mathrm{Y}_{\text {all }} \mathrm{F}$ variants and analyzed for tyrosine-phosphorylation of SLP65 by immunoblotting with a general phospho-tyrosine (4G10) antibody. 
The data presented until now shows that the lack of secondary structure in the central part of SLP65 is compatible with the assembly of the $\mathrm{Ca}^{2+}$ initiation complex. However, this part contains a membrane targeting site, and therefore might not adopt the disordered conformation at all times during SLP65 activation. It is possible that an ordered conformation of this region of the protein exists and is necessary for the membrane anchoring process of SLP65.

\subsubsection{Btk and PLCY2 assemble on SLP65 in a spatially defined manner}

The results presented until now have indicated that the central part of SLP65 between residues 40 and 330 is disordered and can be functionally substituted by a GS-linker under certain conditions. To investigate the flexibility of SLP65 in living cells further, I created another synthetic protein construct, a variant of SLP65 where the Btk and PLCY2 binding sites were swapped. I hypothesized that, given the disordered nature of the central part, the signaling function of this variant would not be affected. Human SLP65 interacts with the Btk SH2 domain via the residue pY96, and with PLCY2 C2 and tandem SH2 domains via residues pY119, pY178 and pY189, respectively (Chiu et al., 2002; Engelke et al., 2013). I generated a construct where the original Btk-binding site was inactivated by an amino acid substitution to phenylalanine (Y96F) and a new Btk-binding site was introduced downstream of the PLCY2-binding sites. The tyrosine residues, mediating the interaction of SLP65 with Btk and PLCY2, are represented in Figure 4.5 in the context of WT SLP65, and in the new construct SLP65 Y96FnewBtk.

SIp65 ${ }^{-1-}$ DT40 cells were retrovirally transduced with either SLP65 WT or Y96FnewBtk. The cells expressing the Y96FnewBtk variant did not respond to BCR stimulation with $\mathrm{Ca}^{2+}$ mobilization, even though the protein was recruited to the plasma membrane (Figure 4.6 A and B). The inability of SLP65 Y96FnewBtk to mount an adequate $\mathrm{Ca}^{2+}$ response could be explained by insufficient tyrosine phosphorylation by Syk and/or by poor recognition of the newly introduced Btk-binding site by the Btk SH2 domain. 


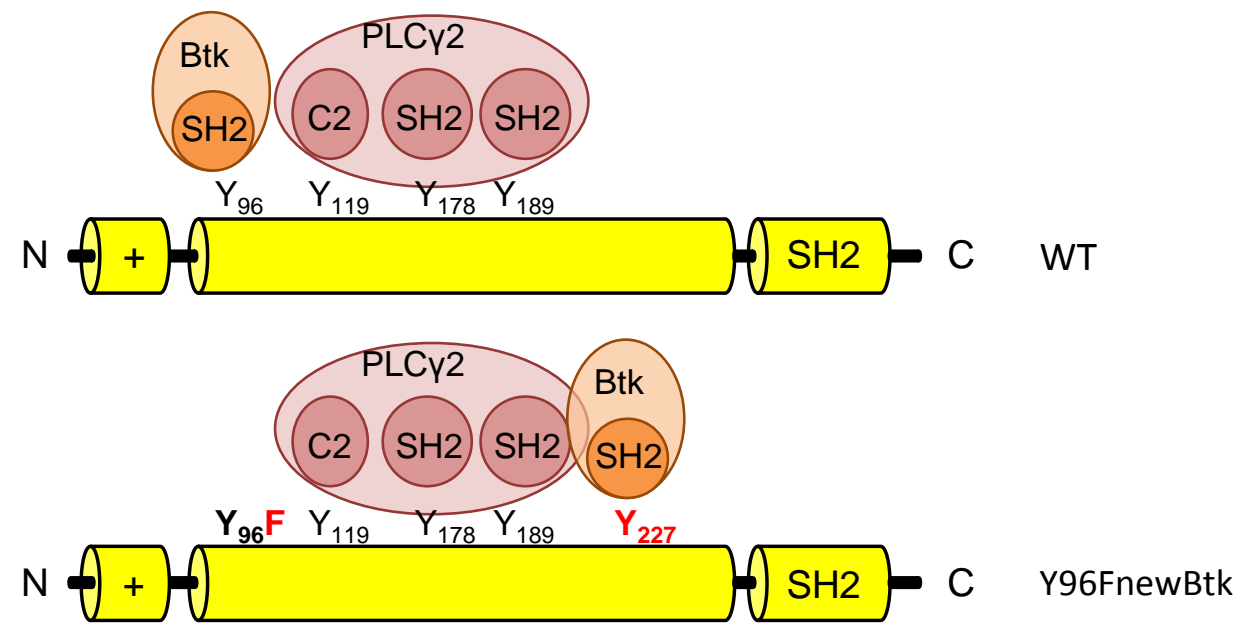

Figure 4.5 Schematic representations of the Btk- and PLCY2-binding sites on SLP65 and the synthetic construct SLP65 Y96FnewBtk.

In the Y96FnewBtk construct the original Btk-binding site was inactivated by a point mutation Y96F and a new Btk-binding site was generated at position 227. The newly introduced Btk-binding site contained the complete phosphotyrosine motif, including a few amino acids upstream and downstream the tyrosine residues to ensure efficient phosphorylation by Syk and recognition by the Btk SH2 domain. The cDNA encoding SLP65 Y96FnewBtk was synthesized by MWG operons and cloned into the pMSCVpuro vector with an $\mathrm{N}$-terminal Citrine-tag.

In order to test the functionality of the new Btk-binding site, I performed pull down experiments with the GST-Itk $\mathrm{SH} 2$ domain fusion protein. This GST-fusion protein was used to pull down SLP65 from lysates of resting and BCR-stimulated cells, expressing either SLP65 WT or Y96newBtk. The SH2 domain of Itk interacts with the phosphotyrosine motif in SLP76, the functional homologue of SLP65. The motif recognized by Itk is identical to that recognized by the $\mathrm{Btk} \mathrm{SH} 2$ domain (YEPP). The Itk $\mathrm{SH} 2$ domain was used instead of the Btk $\mathrm{SH} 2$ domain due to technical difficulties encountered while purifying the GST-Btk $\mathrm{SH} 2$ domain fusion protein. As seen in Figure 4.6 C, both SLP65 WT and Y96FnewBtk were efficiently precipitated with the GST-Itk SH2 domain, but not with the GST-only control, from the lysates of BCR-stimulated cells (lanes 5, 6 and 14, 15, respectively). Moreover, application of phospho-specific antibodies to the immunoblot containing precipitated SLP65 revealed that both variants were efficiently tyrosine phosphorylated upon BCR-stimulation. These data demonstrated that the SLP65 Y96FnewBtk variant was recruited to the plasma membrane and the newly introduced Btk-binding site was phosphorylated and able to interact with Btk/ltk. 
A)

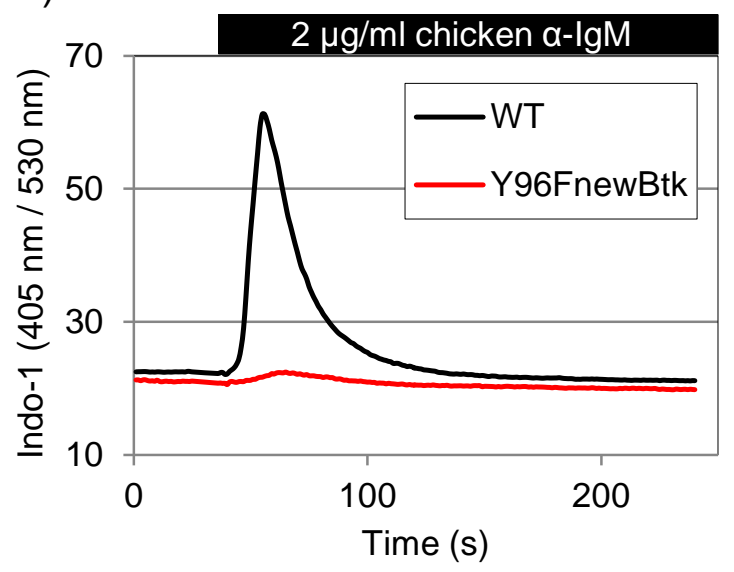

C)

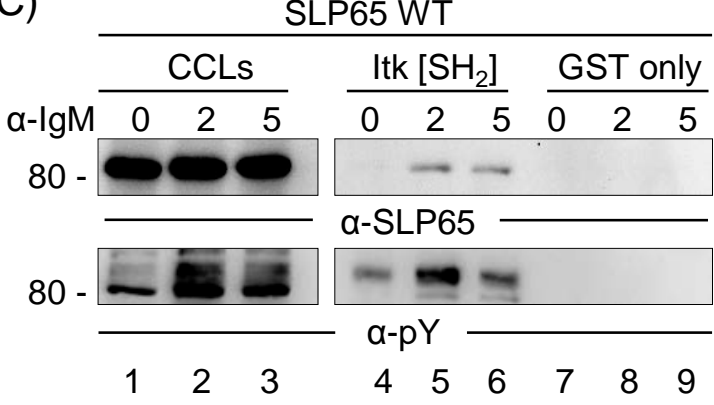

B)

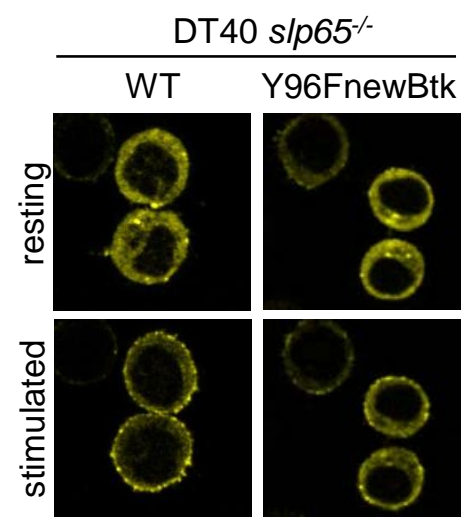

D)

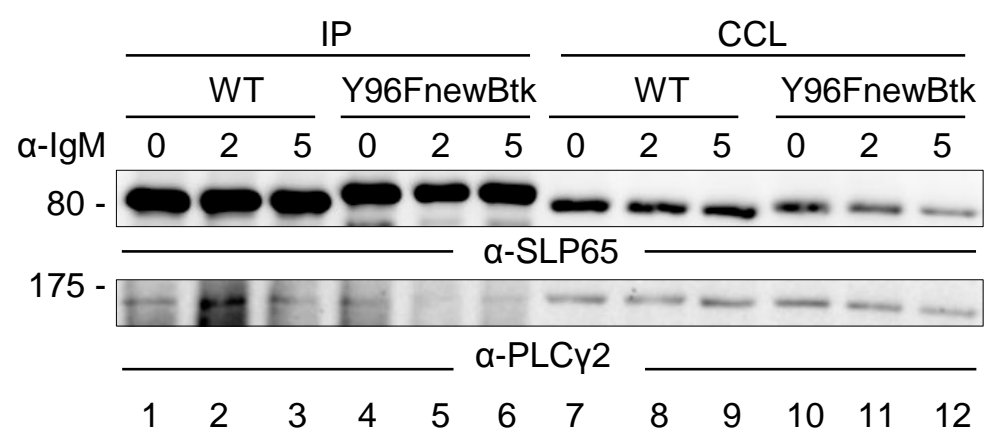

Figure 4.6 Swapping Btk and PLCY2 binding sites in SLP65 renders it unfunctional.

A) DT40 s/p65 $5^{-1-}$ cells were reconstituted with Citrine-tagged SLP65 WT or Y96FnewBtk and analyzed for $\mathrm{Ca}^{2+}$ mobilization by flow cytometry. The $\mathrm{Ca}^{2+}$ flux profiles of cells with equal Citrine expression are shown. B) Subcellular localization and plasma membrane recruitment of Citrinetagged SLP65 variants was analyzed by CLSM. Images before and 3 min after stimulation with 2 $\mu \mathrm{g} / \mathrm{ml} \alpha$-chicken IgM antibody are shown. C) The GST-Itk SH2 domain was expressed and purified from E.coli. It was used to precipitate SLP65 from lysates of resting and BCR-stimulated s/p65 $5^{-1}$ DT40 cells reconstituted with either SLP65 WT or Y96FnewBtk. A pull down experiment with GSTonly protein was performed as a control, to exclude unspecific binding of SLP65 to the GST-tag. D) Citrine-taged SLP65 was immunoprecipitated with an a-GFP antibody from CCLs of resting and BCR-stimulated DT40 cells, expressing either SLP65 WT or Y96FnewBtk. An immunoblot for PLCY2 was performed to detect the association with SLP65. 
The compromised $\mathrm{Ca}^{2+}$ mobilization profile of the Y96FnewBtk variant could still be explained by the inability of this construct to bind PLCY2, since the spacing of the Btk- and PLCY2-binding sites was altered. I therefore performed a co-immunoprecipitation experiment, where Citrine-tagged SLP65 WT or Y96FnewBtk was precipitated from the cellular lysates with an a-GFP antibody, and an immunoblot for PLCY2 was performed to detect its association with SLP65. PLCY2 could only be co-immunoprecipitated with SLP65 WT from cells stimulated via their BCR for 2 min, but not with SLP65 Y96FnewBtk, indicating that the Y96FnewBtk variant of SLP65 indeed cannot bind PLCY2 (Figure 4.6 D, lanes 2 and 4-6). This can be explained by the close proximity of the PLCY2- and the new Btk-binding sites, which doesn't allow for simultaneous binding of Btk and PLCY2. This can in turn account for the weak $\mathrm{Ca}^{2+}$ response, since Btk and PLCY2 need to tether to a single SLP65 molecule in order for phosphorylation and activation of PLCY2 to take place. In summary, these results indicate that the spacing and the orientation of the Btk- and PLCY2- binding sites on SLP65 are important for the assembly of an active $\mathrm{Ca}^{2+}$ initiation complex. The intrinsic disorder in SLP65 is tolerated, or maybe even advantageous for the binding of Btk and PLCY2, but the two enzymes have to be positioned on SLP65 in a defined manner.

\subsection{Structural and functional analysis of the N-terminal region of SLP65}

\subsubsection{Evaluation of previous studies on the SLP65 $\mathrm{N}$ terminus}

We omitted the N-terminal region of SLP65 for the investigation of SLP65 structure by NMR due to technical reasons (low solubility of the full-length construct). However, the $\mathrm{N}$ terminus of SL65 is critical for SLP65 activation and membrane targeting, and the respective mechanism is unknown. I therefore studied the structure and function of this enigmatic part of SLP65.

To analyze the function of the SLP65 N terminus, I reconstituted the s/p65 ${ }^{-\%}$ DT40 cell line with SLP65 variants harboring single point mutations or deletions in this region. The essential N-terminal part of SLP65 can be defined as the first 50 amino acids since the mutant variant lacking these 50 amino acids, SLP65 $\Delta N$, is able to elicit only a very weak $\mathrm{Ca}^{2+}$ response and cannot translocate to the plasma membrane upon BCR stimulation (Figure 4.7). 
A)

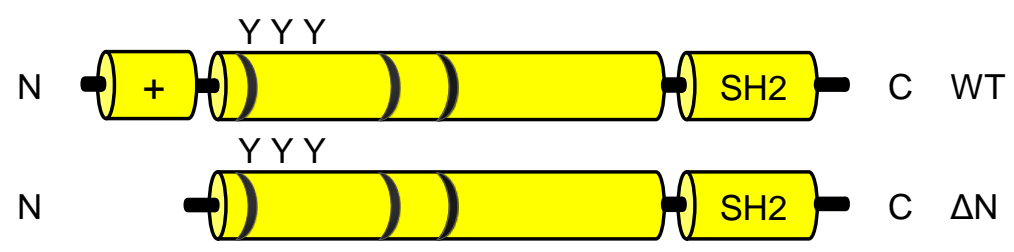

B)

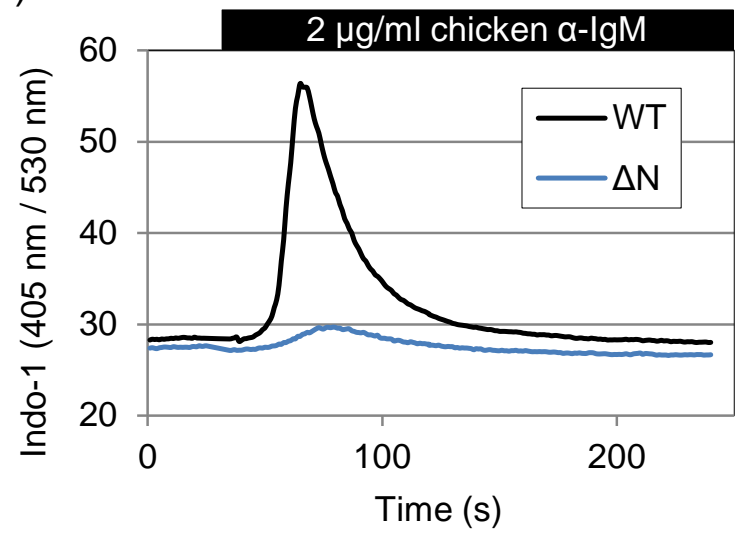

C)

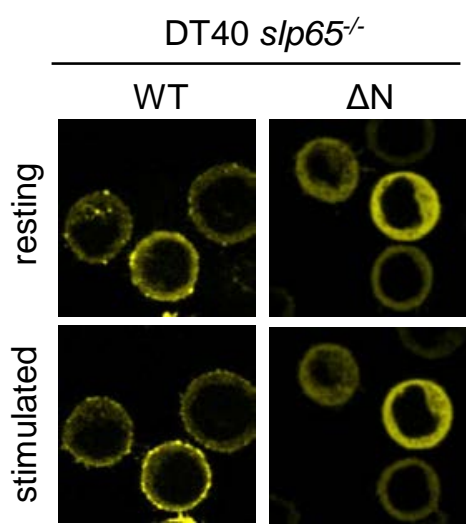

Figure 4.7 The N-terminal region of SLP65 is critical for its function.

A) Schematic representation of SLP65 WT and $\Delta N$. B) s/p65 $5^{-/}$DT40 cells were reconstituted with SLP65 WT or $\Delta \mathrm{N}$ and $\mathrm{Ca}^{2+}$ mobilization was monitored by flow cytometry. The $\mathrm{Ca}^{2+}$ flux profiles of cells with equal Citrine expression are shown. C) Plasma membrane recruitment of SLP65 WT or $\triangle N$ upon BCR-stimulation was analyzed by CLSM. Images before and 3 min after stimulation with $2 \mu \mathrm{g} / \mathrm{ml}$ a-chicken IgM antibody are shown.

The N-terminal region of SLP65 is highly conserved among species. Bioinformatic analysis of the amino acid sequence of this region reveals several potential motifs for interaction with other proteins. One of these motifs is a predicted Calmodulin-binding site. Calmodulin (CaM) is the key regulator of $\mathrm{Ca}^{2+}$ signaling in many cell types. It binds its targets with a protein domain known as "EF hands", which recognizes bulky hydrophobic amino acids such as W, F, I, L or V, with a certain spacing (reviewed by Chin \& Means, 2000). SLP65 has one classical CaM-binding motif of type 1-5-10 at positions 31 to 41 , and two further possible motifs of type 1-8-14 where the last amino acid is shifted by one position (shown in Figure 4.8 A). The potential interaction between SLP65 and CaM has been investigated by $\mathrm{N}$. Herrmann in our lab, who found that this interaction can be detected in vitro by means of pull down assays employing CaM-agarose and the isolated $\mathrm{N}$ terminus of SLP65 (Herrmann, 2009). I attempted to verify this interaction in vivo. I cloned the chicken CaM cDNA and expressed the Citrine-tagged CaM in DT40 WT cells. CLSM imaging analysis revealed that CaM is localized mainly in the cytosol of resting $B$ 
cells. I also monitored CaM localization 1, 3 and 5 min after cell stimulation with $\alpha$-IgM antibodies. Interestingly, the localization of CaM did not change upon BCR stimulation (Figure $4.8 \mathrm{~B}$ ). Nor did the overexpression of CaM in DT40 cells influence the $\mathrm{Ca}^{2+}$ flux upon BCR ligation (Figure $4.8 \mathrm{C}$ ).

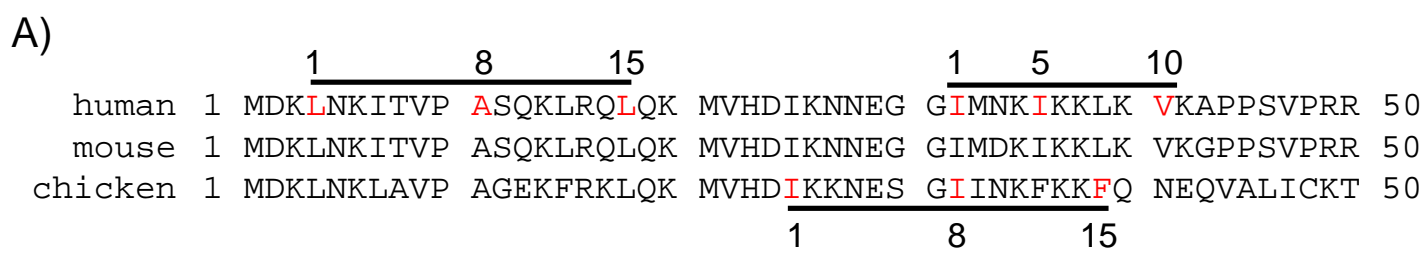

B)

DT40 WT Cit-CaM

resting stimulated 1' stimulated 3' $^{\prime}$ stimulated 5'

C)

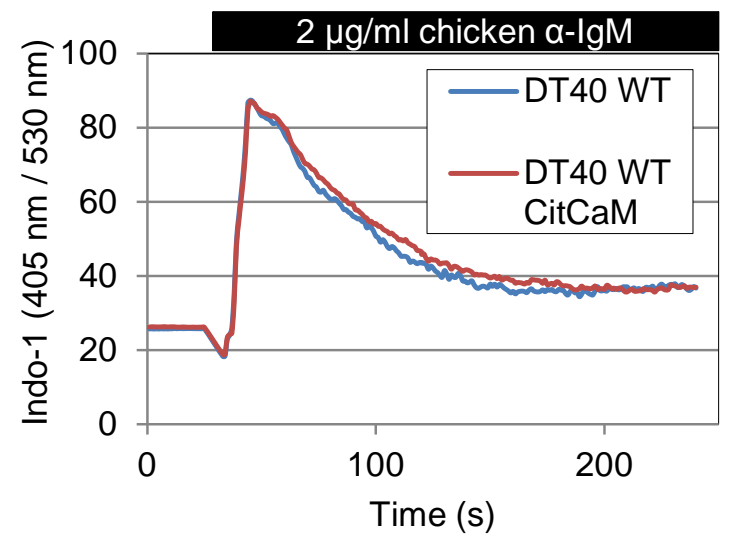

D)

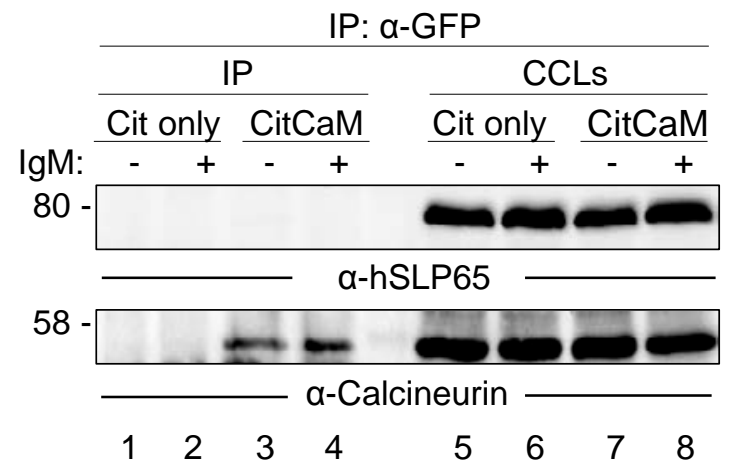

Figure 4.8 The putative SLP65-Calmodulin interaction cannot be observed in vivo and the overexpression of CaM does not affect $\mathrm{BCR}$-induced $\mathrm{Ca}^{2+}$ mobilization.

A) Amino acid sequence of human, mouse and chicken SLP65 with the putative CaM-binding sites in the $\mathrm{N}$ terminus as predicted by the Calmodulin Target Database. B) DT40 WT cells were retrovirally transduced with Citrine-tagged chicken $\mathrm{CaM}$ and the localization of $\mathrm{CaM}$ was monitored by CLSM. Images before and 1, 3 or 5 min after stimulation with $2 \mu \mathrm{g} / \mathrm{ml} \alpha$-chicken IgM antibody are shown. C) Intracellular $\mathrm{Ca}^{2+}$ flux was monitored by flow cytometry in DT40 WT cells and DT40 WT cells transduced with Citrine-CaM. D) DT40 s/p65 $5^{-1}$ cells, previously transfected with OSSLP65, were retrovirally transduced with either Citrine only or Citrine-tagged CaM. CCLs were prepared from resting and BCR-stimulated cells and subjected to immunoprecipitation with $2 \mu \mathrm{g} \alpha-$ GFP antibody. Immunoblot with a-SLP65 and a-Calcineurin antibodies was performed to detect the interaction with CaM. 
Finally, I performed a protein immunoprecipitation experiment and pulled down CitrineCaM with the help of an a-GFP antibody. An immunoblot for SLP65 was subsequently performed in order to detect a potential interaction between the two proteins. I used DT40 s/p65\% cells reconstituted with human One-Strep tagged SLP65 and additionally transfected with Citrine-CaM or Citrine only (in order to exclude unspecific binding of proteins to the Citrine tag). As can be seen in Figure $4.8 \mathrm{D}$, SLP65 could not be coimmunoprecipitated with CaM (lanes 3-4) from the lysates of resting or stimulated cells. However, a known interaction partner of CaM, Calcineurin, was successfully coimmunoprecipitated (Figure $4.8 \mathrm{D}$, lanes 3-4), indicating that the pull down has worked, and that the Cit-CaM fusion protein is functional and can bind its ligands. The lysis buffer used in this experiment contained $10 \mu \mathrm{M} \mathrm{CaCl}{ }_{2}$ corresponding to a sufficiently high concentration of $\mathrm{Ca}^{2+}$ to ensure that $\mathrm{CaM}$ is able to interact with its targets.

In conclusion, the interaction of SLP65 with CaM could not be detected in living cells. Moreover, overexpression of CaM did not have a positive or negative regulatory effect on early BCR signaling indicating that the potential interaction of CaM with the SLP65 N terminus doesn't play a major role for the activation and membrane recruitment of SLP65. Another motif proposed to exist in the SLP65 N terminus, is the so called "leucine-zipper" motif, as suggested by Koehler et al. The arrangement of hydrophobic amino acids in this region indeed reminds a classical heptad repeat pattern characteristic of leucine zippers (Figure $4.9 \mathrm{~A}$ ), where the positions $a$ and $d$ are occupied by hydrophobic, and positions $e$ and $g$ by charged residues. In the study from Koehler et al, a substitution of the isoleucine residue central to the leucine zipper motif with a glutamate residue (I25E) was shown to abolish the membrane localization and function of SLP65 (Kohler et al., 2005). I25E is a drastic amino acid substitution from a biochemical point of view, since glutamate (E) has a hydrophilic and negatively charged side chain which could distort the contrasting hydrophobic and positively charged environment created by other residues of the SLP65 $\mathrm{N}$ terminus. I therefore expressed additional point mutants of SLP65, I25K and I25A, in DT40 s/p65 $5^{-1}$ cells and analyzed their subcellular localization and $\mathrm{Ca}^{2+}$ mobilization profiles. I used substitutions to alanine or lysine in order to preserve the hydrophobic nature or the overall positive charge of the $\mathrm{N}$ terminus, respectively. As shown in Figure $4.9 \mathrm{~B}$ and $\mathrm{C}$, the $\mathrm{Ca}^{2+}$ mobilization of these mutants was only marginally impaired and the plasma membrane recruitment of SLP65 upon BCR stimulation was readily observed. Thus, inactivation of the predicted leucine zipper via amino acid substitutions for other aliphatic residues did not interfere with SLP65 function. 
A) abcdef gabcdefgab cdefgabcde fg

human 1 MDKLNKITVP ASQKLRQLQK MVHDIKNNEG GIMNKIKKLK VKAPPSVPRR 50 mouse 1 MDKLNKITVP ASQKLRQLQK MVHDIKNNEG GIMDKIKKLK VKGPPSVPRR 50 chicken 1 MDKLNKLAVP AGEKFRKLQK MVHDIKKNES GIINKFKKFQ NEQVALICKT 50

B)

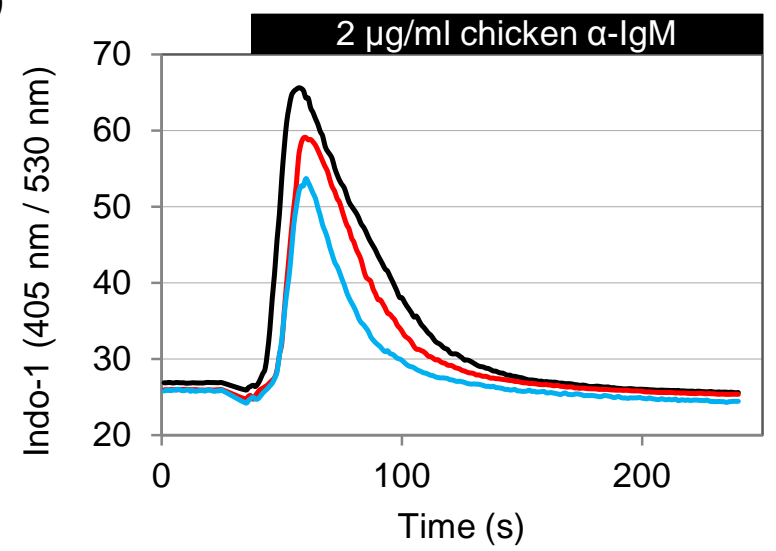

D)

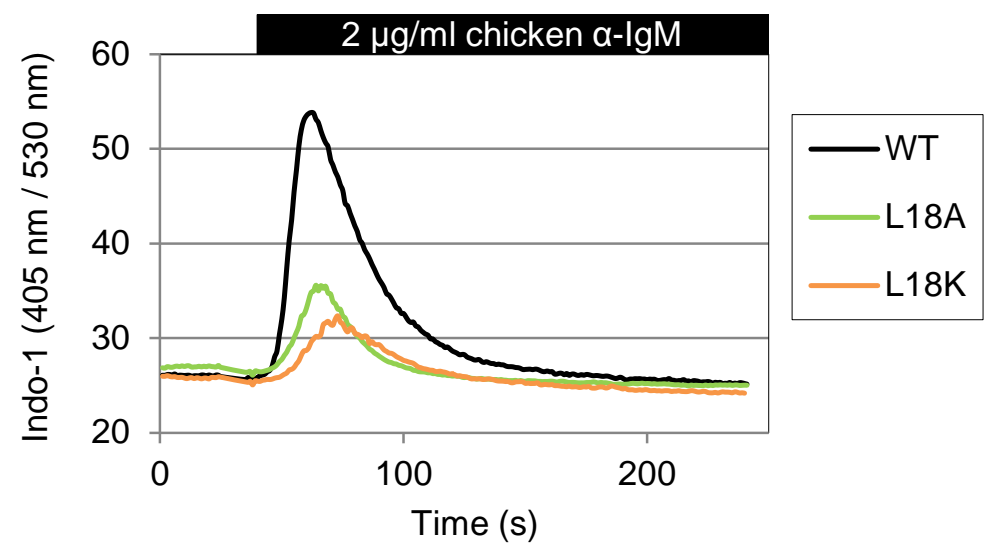

C) $\quad \frac{\mathrm{DT} 40 \mathrm{~s} / \mathrm{p} 65^{-/}}{\mathrm{I} 25 \mathrm{~K}}$

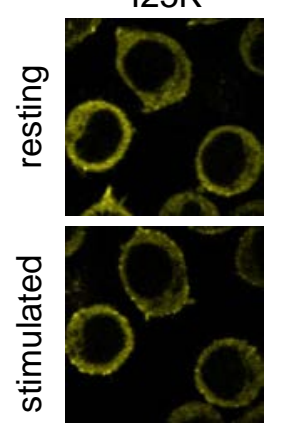

E)

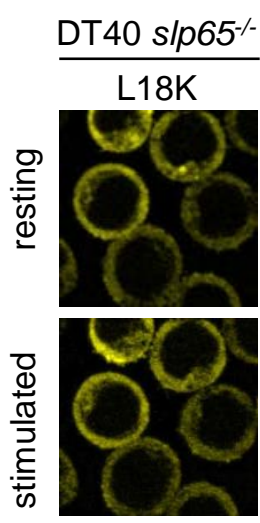

Figure 4.9 Single amino acid exchanges in the putative leucine zipper motif of SLP65 differentially affect $\mathrm{BCR}$-induced $\mathrm{Ca}^{2+}$ mobilization.

A) Amino acid sequence of human, mouse and chicken SLP65, and the putative leucine zipper motif the $\mathrm{N}$-terminal region are shown. B) and D) DT40 s/p65 cells were retrovirally transduced with constructs encoding Citrine-tagged SLP65 WT, 125A, 125K, L18K or L18A and $\mathrm{Ca}^{2+}$ mobilization was monitored by flow cytometry. The $\mathrm{Ca}^{2+}$ flux profiles of cells with equal Citrine expression are shown. C) and E) Subcellular localization and plasma membrane recruitment of Citrine-tagged SLP65 I25K and L18K was analyzed by CLSM. Images before and 3 min after stimulation with $2 \mu \mathrm{g} / \mathrm{ml} \alpha$-chicken IgM antibody are shown. 
I extended the mutational analysis to another hydrophobic residue in the SLP65 N terminus - L18. In this case, substitution of L18 to A or $K$ strongly reduced the BCRinduced $\mathrm{Ca}^{2+}$ mobilization (Figure 4.9 D). Moreover, the plasma membrane recruitment of SLP65 L18A and L18K could not be detected (Figure 4.9 E). L18 is therefore, in contrast to 125 , indispensable for the membrane targeting of SLP65.

To conclude, my data excludes a functional leucine zipper motif in the $\mathrm{N}$ terminus of SLP65. Besides, previous studies from our lab elucidated the interactome of SLP65 in resting and stimulated $B$ cells by quantitative mass spectrometry. In this study more than 30 binding partners of SLP65 were identified, however no ligand for the putative leucine zipper motif was found (Oellerich et al., 2011). The dependence of SLP65 function on L18, however, could point to a general requirement for hydrophobic residues in the SLP65 $\mathrm{N}$ terminus.

\subsubsection{The $\mathrm{N}$ terminus of SLP65 is a novel lipid-binding module which targets SLP65 to vesicles in resting $B$ cells}

To further investigate the function of the $\mathrm{N}$ terminus in membrane targeting of SLP65, we used CLSM imaging and cell fractionation experiments and analyzed SLP65 distribution in living cells. Dr. M. Engelke has observed that Citrine-tagged WT SLP65 is localized in the cytosol as well as in intracellular dot-like structures of resting DT40 cells. Interestingly, these dots disappeared shortly after BCR stimulation, which coincided with the appearance of SLP65 at the plasma membrane. He performed a series of colocalization experiments employing Citrine-tagged SLP65 and Cerulean-tagged vesicular markers which were co-expressed in the same cell. His analysis revealed that SLP65 colocalizes with the exosomal vesicular markers quinacrine and Vamp7 (vesicle-associated membrane protein 7) in resting DT40 cells. In contrast, no colocalization of SLP65 with Golgi or endosomal markers was observed. Colocalization with quinacrine and Vamp7 depended on the N-terminal region of the protein (Engelke et al., 2014 and Figure 4.10 A).

In order to confirm the membrane association of SLP65 in resting cells, cellular fractionation assays were performed by J. Kühn. In these experiments, DT40 s/p65 ${ }^{-1-}$ cells reconstituted with either WT or N-terminal variants of SLP65 $\Delta \mathrm{N}$ or L18K were lysed by the nitrogen cavitation method in a hypotonic buffer without any detergent in order to preserve the intracellular membranes. The lysates were then fractionated on a sucrose gradient. Fractions were taken for analysis and the SLP65 distribution in the sucrose gradient was analyzed by immunoblotting. As shown in Figure 4.10 B, WT SLP65 was 
largely found in the cytosolic fractions. However some SLP65 was also present in the interphase between fractions containing $35 \%$ and $45 \%$ sucrose, corresponding to a compartment containing membranes of high density. In contrast, the signaling incompetent mutants of SLP65, $\Delta \mathrm{N}$ and L18K, were found exclusively in the cytosolic fractions. In these experiments, Akt kinase was used as a cytosolic marker.

A)

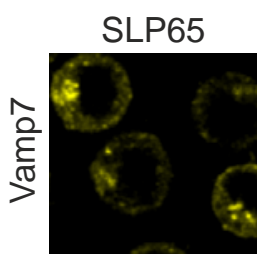

vesicle

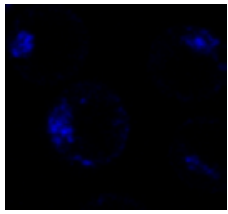

colocalization
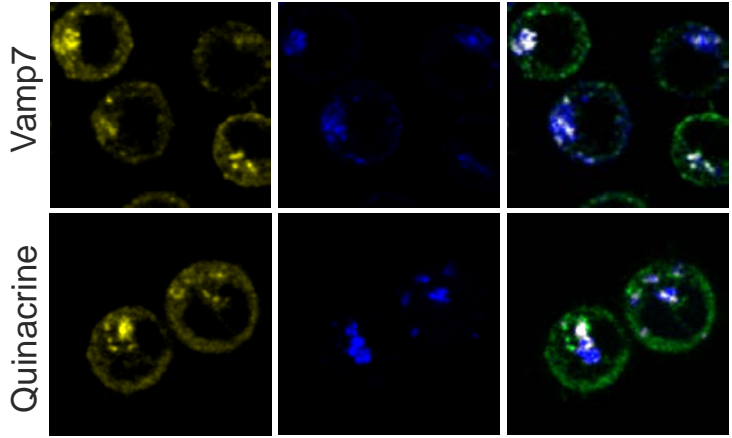

B)
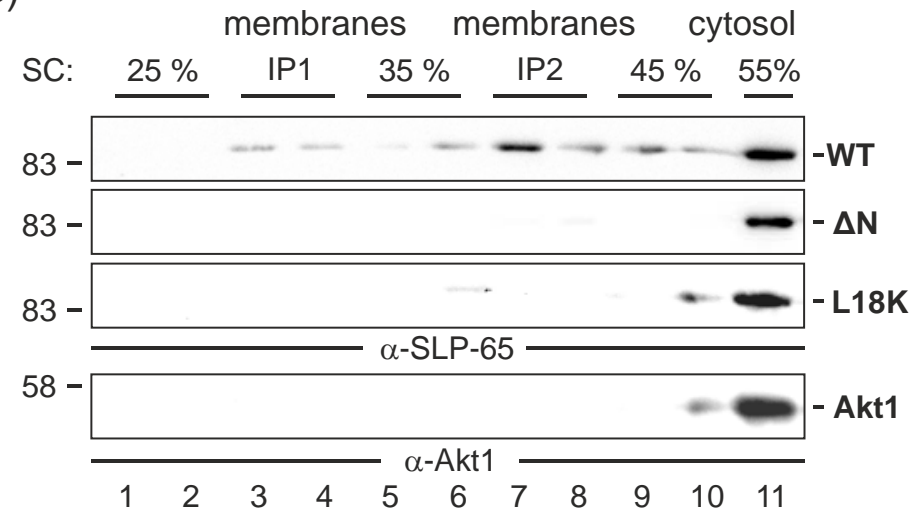

Figure 4.10 SLP65 colocalizes with vesicular markers quinacrine and Vamp7 dependent on its $\mathbf{N}$ terminus.

A) DT40 s/p65 cells were retrovirally transduced with Citrine-tagged SLP65 and Cerulean-tagged vesicular markers. Colocalization of SLP65 WT with Vamp7 and quinacrine was assessed by CLSM and is indicated by white dots in the "colocalization" panel. Imaging and colocalization analysis was performed by Dr. M. Engelke. B) DT40 s/p65 cells reconstituted with SLP65 WT, $\Delta N$ or L18K were lysed by the nitrogen cavitation method and subjected to subcellular fractionation on a discontinuous sucrose gradient by ultracentrifugation. SLP65 distribution in cytosolic and membrane fractions was analyzed by immunoblotting. Immunoblot for the cytosolic protein Akt1 was performed as a control. The subcellular fractionation experiment was performed by J. Kühn. 
Collectively, these data indicate that SLP65 associates with vesicular membranes in resting $\mathrm{B}$ cells dependent on its $\mathrm{N}$ terminus. This finding is in accordance with the data by Koehler et al, who also detected a basal level of membrane association of SLP65 in resting $B$ cells and when ectopically expressed in other cell types, even though plasma membrane and not vesicular localization was reported (Kohler et al., 2005).

The imaging and cell fractionation experiments have indicated that two pools of SLP65 exist in resting DT40 cells - the cytosolic pool and the vesicular pool. I wondered whether there is a clear separation between these two pools or whether protein exchange can take place between the cytosol and vesicles. I therefore analyzed the mobility of SLP65 in vivo with a Fluorescence Recovery after Photobleaching (FRAP) assay. For these experiments, DT40 s/p65/- cells reconstituted with Citrine-tagged SLP65 were used. First, a series of images of a single cell was taken. Subsequently, a region of interest encompassing one bright dot/speckle, presumably corresponding to vesicle-associated SLP65, was chosen. Next, this region was photo-bleached with a strong laser pulse. Immediately after the bleach-pulse a series of post-bleach images was taken in order to monitor the fluorescence recovery in real time. In FRAP experiments, slow and incomplete fluorescence recovery indicates the presence of a large immobile fraction of the fluorophore - in this case, Citrine-SLP65. This would mean SLP65 is stably associated with the vesicle and therefore unable to exchange with the cytosol. Fast and complete fluorescence recovery, on the other hand, implies absence of an immobile fraction and free diffusion of SLP65 between the cytosolic and vesicular pools.

As can be seen in Figure 4.11, rapid fluorescence recovery of Citrine-SLP65 was observed, indicating that there's a steady exchange between the cytosolic and the membrane-associated fractions. For comparison, I photo-bleached Cerulean-tagged Vamp7 expressed in DT40 WT cells and monitored its fluorescence recovery. Fluorescence recovery of Vamp7 was slow and incomplete as expected for a transmembrane protein. 


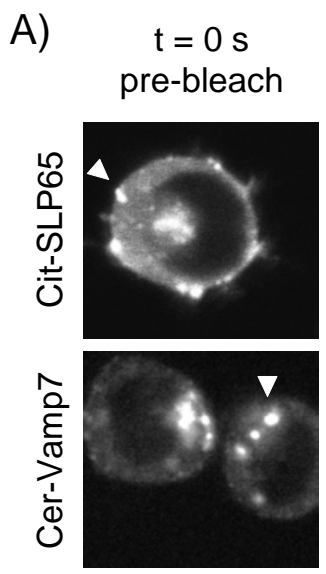

$$
\begin{aligned}
& t=5 s \\
& \text { bleach }
\end{aligned}
$$
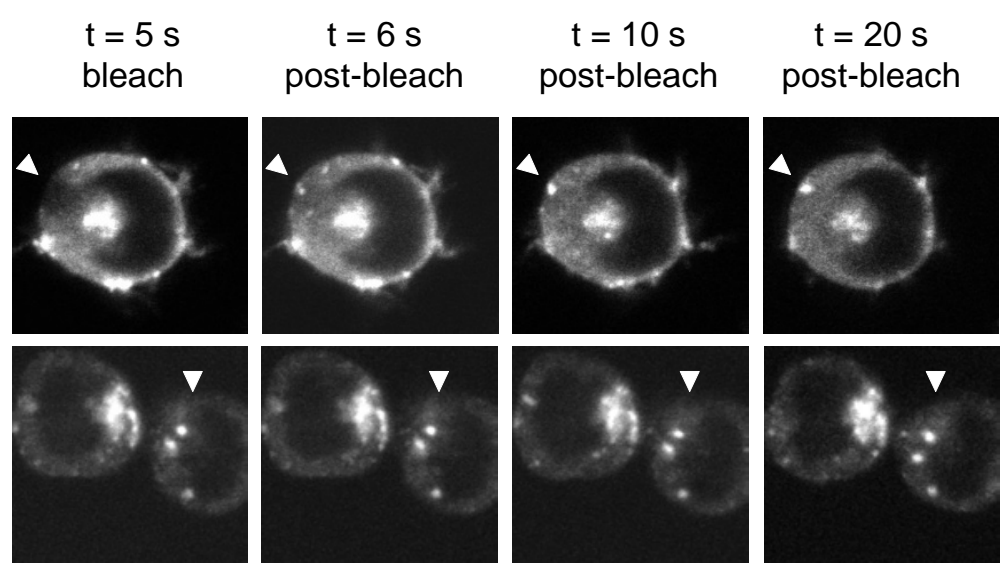

B)

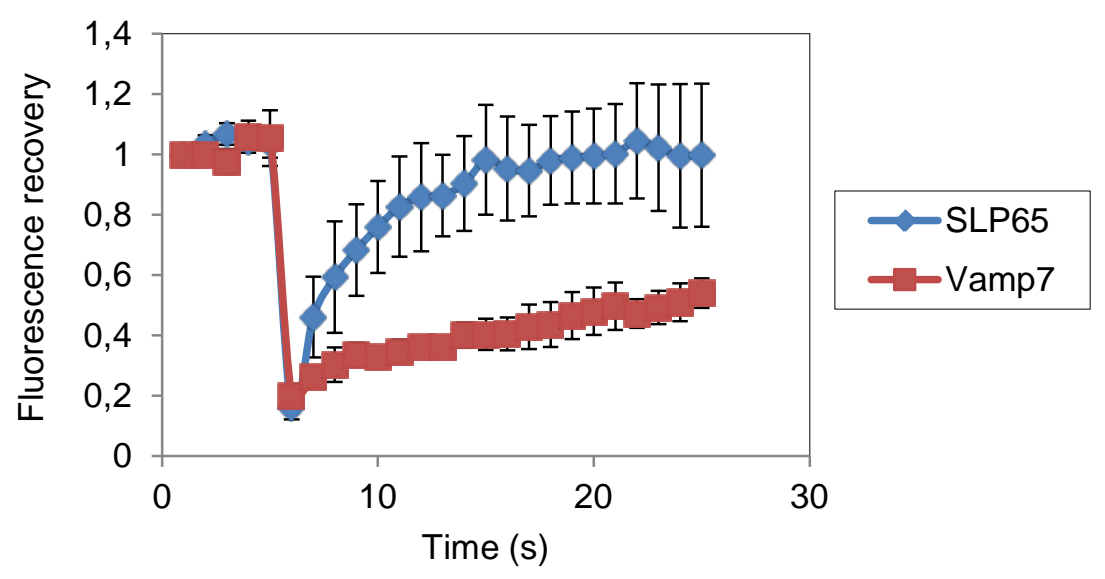

Figure 4.11 Cytosolic and membrane fractions of SLP65 are in constant exchange.

A) FRAP was monitored in DT40 s/p65 $5^{-/}$cells reconstituted with human Citrine-SLP65 (upper panel) or DT40 WT cells transduced with Cerulean-Vamp7. The region of interest, chosen for photo-bleaching, is indicated by white arrows. 5 images were taken before, and 20 images after the photobleaching. B) Quantification of fluorescence in the region of interest before and after the bleach pulse is shown. $n=7, \pm \mathrm{SD}$ (standard deviation).

Our data showed that the $\mathrm{N}$ terminus mediates reversible association of SLP65 with vesicles. I therefore asked the question: what is the molecular nature of this membrane association and what is the ligand for the SLP65 N terminus? Proteomic approaches, previously applied in the lab, did not identify a protein ligand for this part of SLP65 (Oellerich et al., 2011). Therefore, I tested whether SLP65 could bind lipid components of intracellular membranes with a liposome floatation assay, established in the lab of $\mathrm{K}$. Kühnel (Busse et al., 2013). In this method, unilamellar liposomes of the desired lipid composition are prepared and incubated with the recombinant protein of interest. 
Subsequently, the protein-liposome mixture is overlaid with a Nycodenz gradient and subjected to ultracentrifugation. During ultracentrifugation the liposomes float up to the top of the gradient due to their low density. The protein stays at the bottom of the tube if it doesn't bind to liposomes. If binding takes place, the protein also migrates up to the top of the tube (Figure $4.12 \mathrm{~B}$ ). For this assay, N-terminally $\mathrm{His}_{6}$-tagged variants of SLP65, WT, $\Delta \mathrm{N}$ and L18K, purified on Ni-NTA affinity chromatography columns were used (Figure $4.12 \mathrm{~A})$.

A)

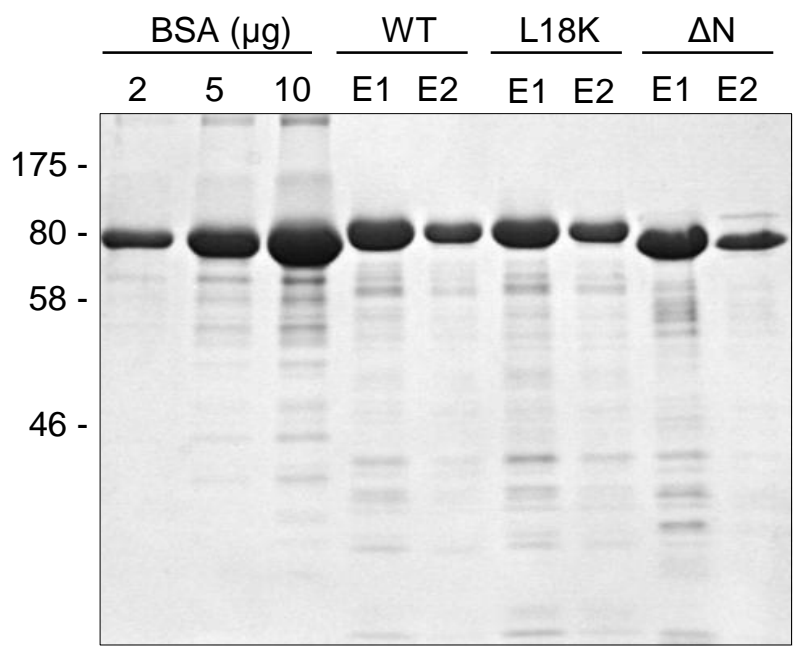

B)

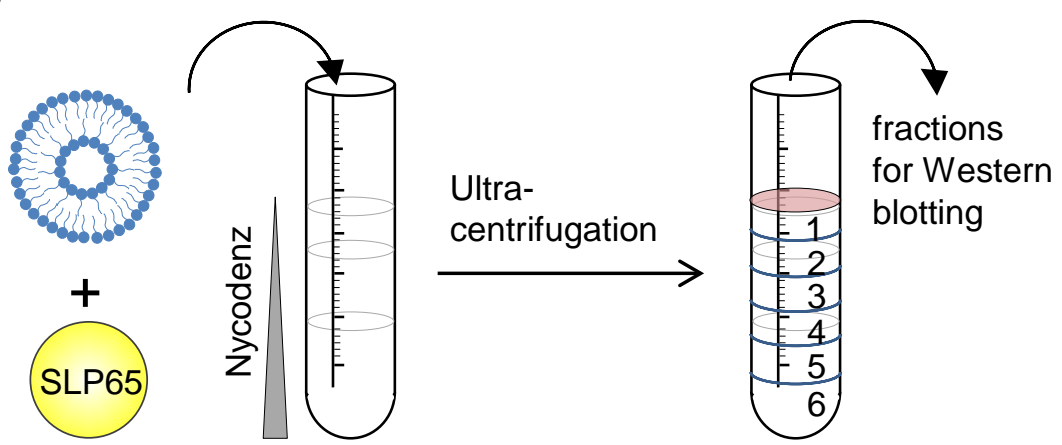

Figure 4.12 The purification of His-tagged SLP65 and an overview of the liposome floatation method.

A) $\mathrm{His}_{6}$-tagged human SLP65 variants were expressed in E.coli and purified on Ni-IDA columns. Two elution fractions were collected (E1 and E2) and $5 \mu \mathrm{L}$ of the eluted protein was loaded on the gel together with a known amount of BSA standard for quantification. The gel was stained with Coomasie for protein visualization. B) Schematic representation of the liposome floatation method. The protein-liposome mixture is overlaid with a Nycodenz gradient and subjected to ultracentrifugation. Protein distribution is analyzed by Western blotting. 
Small unilamellar liposomes (SUVs) were prepared with the "detergent removal" technique. Since previous work by N. Herrmann has indicated that the SLP65 N terminus could bind several PIP species (Herrmann, 2009), PIPs were included in the liposome composition. I therefore prepared liposomes consisting of $70 \%$ phosphatidylcholine (PC), 23\% phosphatidylethanolamine (PE), 5\% PIP (either phosphatidyl inositol 3-phosphate, phosphatidyl inositol 4-phosphate or phosphatidyl inositol 5-phosphate) and 2\% TexasRed (TR)-labeled PE, which was used as a tracer. As a control, liposomes not-containing any PIPs, and therefore consisting of only PC (75\%) and PE (23\% PE and 2\% TR-PE) were prepared.

As shown in Figure 4.13 A (left panel), WT SLP65 associated with the liposomes independent of lipid composition and was found in the topmost two fractions. SLP65 $\triangle N$, in contrast, was only found in the lowermost fractions, indicating that it did not bind to the liposomes (Figure 4.13 A, right panel). This data showed that SLP65 can associate with neutral liposomes, composed of only PC and PE, dependent on its $\mathrm{N}$ terminus. Next, I tested the SLP65 variant L18K for binding to PC:PE liposomes. As shown in Figure 4.13 B (right panel), similarly to SLP65 $\Delta \mathrm{N}$, SLP65 L18K did not float with the liposomes. In order to exclude unspecific binding of SLP65 to TR, which is a fluorescent dye with a negative charge and could therefore bind to positively charged SLP65 via unspecific electrostatic interactions, I used liposomes containing Oregon-Green (OG)-labeled PE. WT SLP65 bound equally well to both TR- and OG-labeled liposomes, while L18K did not associate with the liposomes independent of the dye type (Figure $4.13 \mathrm{~B}$ ), indicating that liposome binding by SLP65 is specific.

Altogether, these data show that the SLP65 $\mathrm{N}$ terminus is a lipid-binding motif which can mediate interaction of SLP65 with liposomes in vitro. Deletion of the N-terminal region or the single amino acid substitution of L18 to K abolished the interaction of SLP65 with SUVs. In vivo, the SLP65 mutants $\Delta \mathrm{N}$ and L18K also do not associate with intracellular membranes as shown with the cell fractionation assays. Moreover, these variants of SLP65 are signaling incompetent, i.e. they cannot initiate $\mathrm{Ca}^{2+}$ signaling. Therefore I conclude that lipid binding is important for SLP65 activation and plasma membrane translocation. 
A)
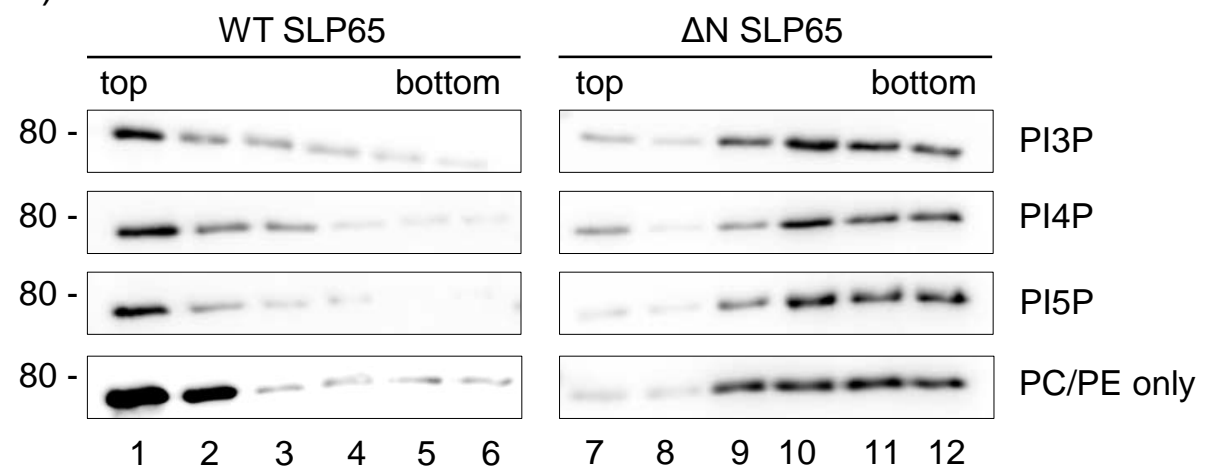

B)
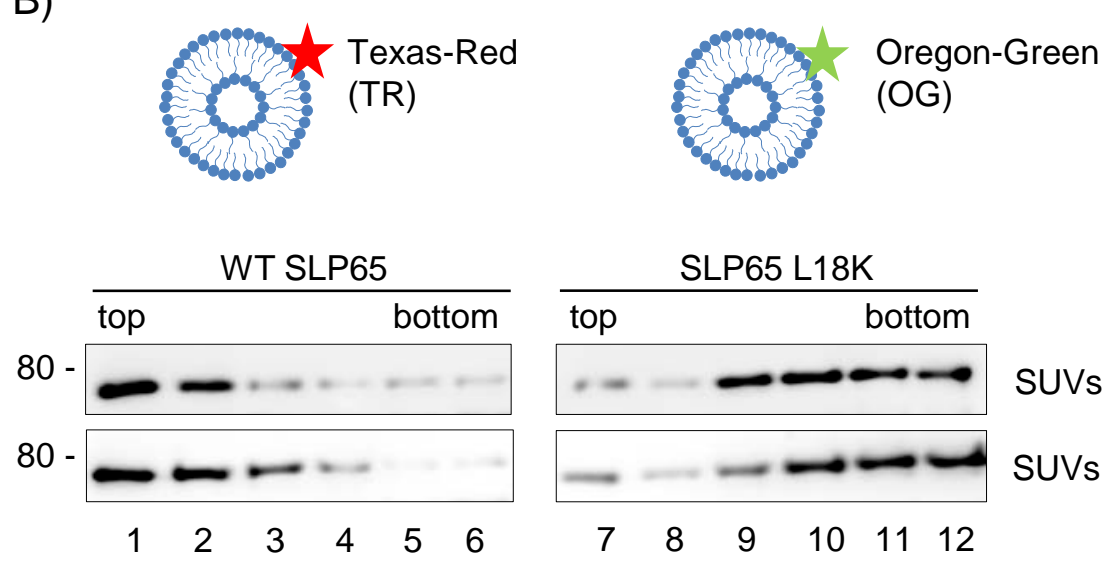

SUVs with TR

SUVs with OG

Figure 4.13 SLP65 binds to neutral liposomes dependent on its $\mathbf{N}$ terminus.

A) Liposome floatation assay was performed with recombinant His ${ }_{6}$-tagged WT and $\Delta \mathrm{N}$ and SUVs consisting of PC, PE and PIP; or PC and PE only. Representative immunoblots developed with an a-SLP65 antibody are shown. B) Liposomes of the same lipid composition, but with different fluorescent labels - either TR or OG were prepared. Liposome floatation assay was performed with WT and L18K SLP65 and SUVs labeled with either TR (upper panel) or OG (lower panel). Representative immunoblots, developed with an a-SLP65 antibody, are shown.

\subsubsection{The $\mathrm{N}$ terminus of SLP65 binds preferentially to negatively charged membranes and may recognize membrane curvature}

Next, I aimed at characterizing the newly identified interaction of the SLP65 N terminus with lipids. To study the specificity of lipid binding, I performed liposome floatation experiments with liposomes of different size and lipid composition. Since the SLP65 N terminus is rich in positively-charged amino acids, such as lysines and arginines, I hypothesized that it would preferentially bind to negatively charged membranes. I hence performed floatation assays with liposomes containing a negatively charged lipid 
phosphatidylserine (PS) in their composition. In order to compare the affinities of SLP65 for negatively charged versus neutral liposomes, I increased the amount of protein used in my floatation experiments. By using 5 times more protein, I could reach a condition where not all SLP65 was bound to PC:PE liposomes and some protein remained in the nonliposomal fractions. I then performed the floatation experiment with the same protein:lipid molar ratio, but used negatively charged liposomes. As shown in Figure 4.14, at higher protein:lipid ratios (left panel) incomplete binding of SLP65 to neutral SUVs was observed, whereas almost all protein was bound to negatively charged SUVs. This experiment shows that SLP65 has a higher affinity for PS-containing liposomes, suggesting that the binding of the SLP65 N terminus to membranes is mediated, at least in part, by electrostatic interactions.

A)
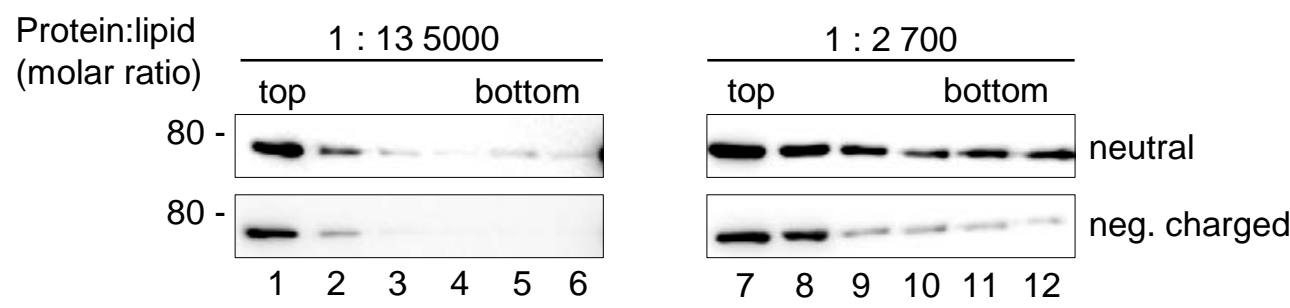

B)
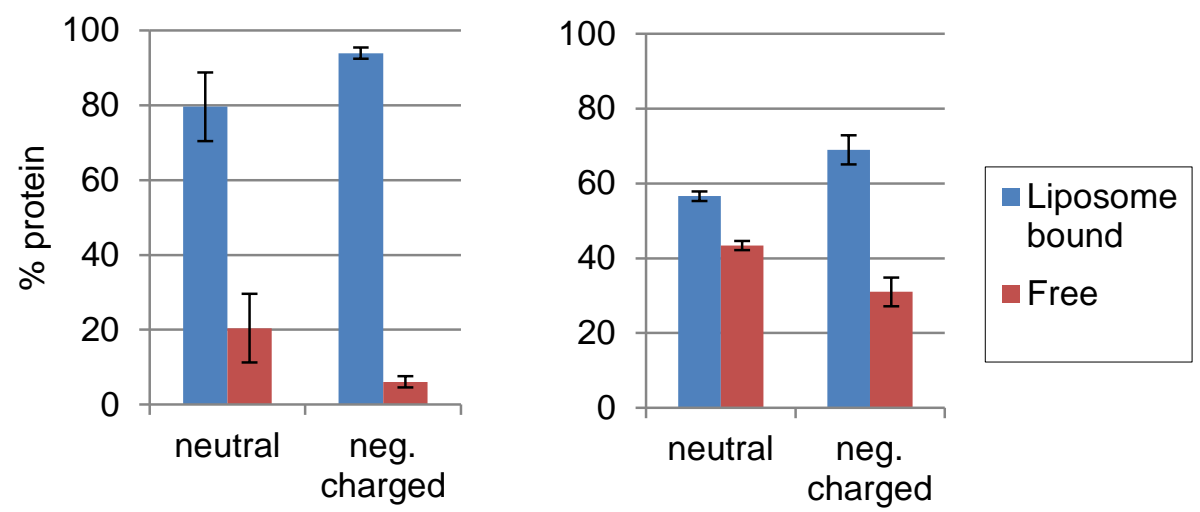

Figure 4.14 SLP65 preferentially binds to negatively charged liposomes.

A) Either $1 \mu \mathrm{L}$ (left panel) or $5 \mu \mathrm{L}$ (right panel) SLP65 was incubated with PC:PE (75:25) or PC:PE:PS (65:25:10) liposomes and a liposome floatation experiment was performed. Representative immunoblots, developed with an a-SLP65 antibody, are shown. B) Quantification of the band intensities in the top two fractions (liposome-bound) and lowermost four fractions (free protein) is shown. $n=3 \pm S D$. 
The membranes enclosing different organelles in a cell have a highly variable lipid composition, which gives them different physical and chemical properties. These different parameters, such as electrostatics, lipid packing and membrane curvature, are recognized by peripheral membrane proteins, which adhere to membranes from the cytosol (Bigay \& Antonny, 2012). My data until now has indicated that SLP65 can recognize membranes based on their charge. Next, I asked the question whether it could also recognize membrane curvature.

Therefore, in addition to SUVs, prepared by the "detergent removal" method, I also prepared Large Unilamellar Vesicles (LUVs) via the "extrusion" method. This method generates vesicles of a certain diameter by mechanically extruding a lipid suspension through a series of filters of different pore size. By extruding a suspension of multilamellar vesicles (MLVs) through 0.4 and $0.1 \mu \mathrm{m}$ filters, I prepared liposomes of $\sim 60 \mathrm{~nm}$ radius. The sizes of LUVs and SUVs were monitored by Dynamic Light Scattering (DLS). Both liposome preparations were homogenous in size and the average geometric radius was determined to be $18 \mathrm{~nm}$ for SUVs and $60 \mathrm{~nm}$ for LUVs (Figure $4.15 \mathrm{~A}$ ). I then tested the affinity of SLP65 for these two types of vesicles by means of floatation assays. Prior to use in the floatation experiment, the concentration of the liposomes was adjusted by measuring the absorbance of TR at $590 \mathrm{~nm}$ and diluting the more concentrated vesicle suspension with the appropriate buffer. As shown previously, SLP65 readily bound to SUVs consisting of PC and PE. Interestingly, no binding of SLP65 to LUVs of the same lipid composition was observed (Figure $4.15 \mathrm{~B}$ and $\mathrm{C}$ ). As a control, a floatation experiment with Hsv2, a protein which binds to liposomes independent of curvature, was performed. Hsv2 recognizes PI3P, therefore $5 \%$ of this lipid was included in the lipid composition of both SUVs and LUVs (Busse et al., 2013). Hsv2 associated with both SUVs and LUVs, indicating that both type of liposomes were intact, and are in principle able to accommodate peripheral membrane proteins (Figure 4.15 D). 
A)

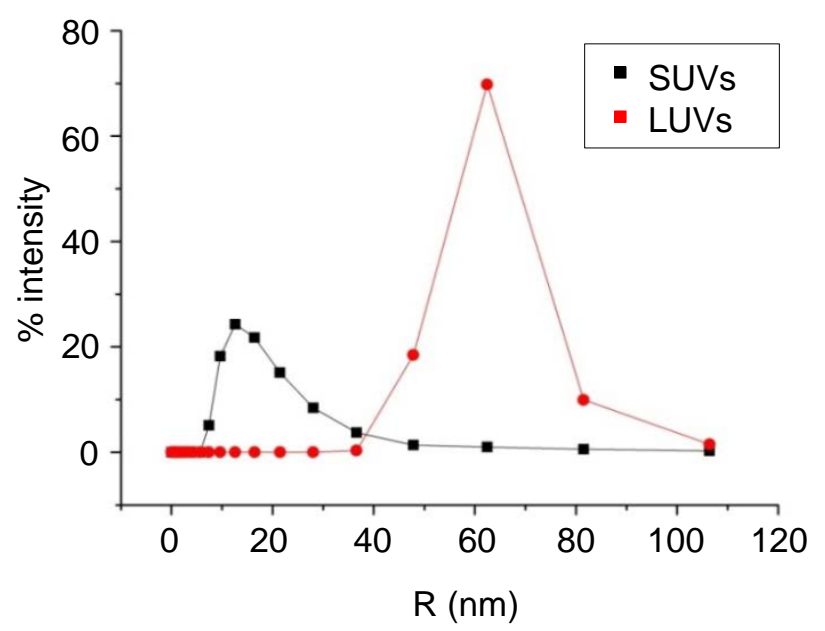

B)

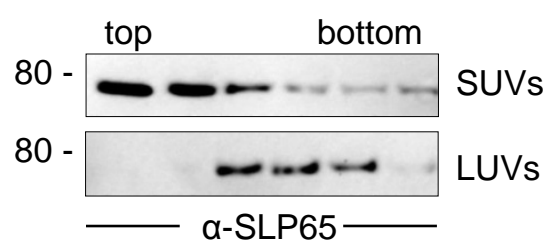

$1 \quad 2 \quad 3 \quad 4 \quad 56$
C)

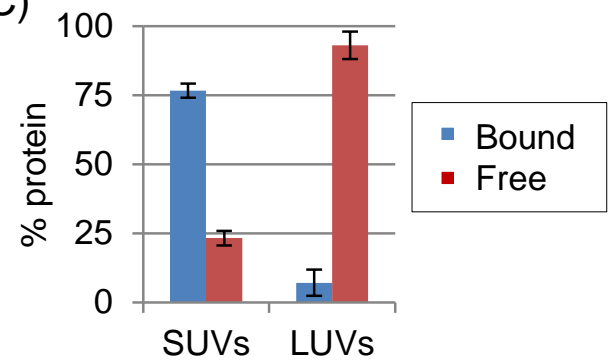

D)

PC, PE, 5\% PI3P
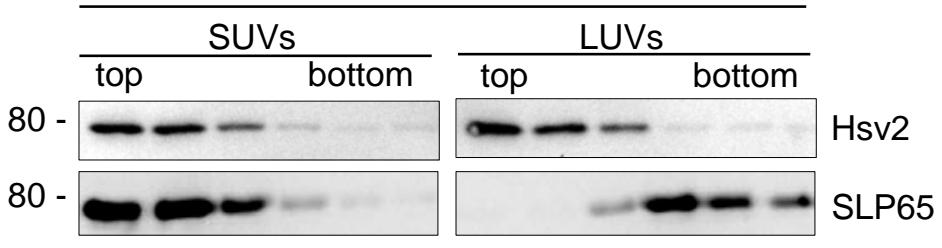

$\begin{array}{llllllllllll}1 & 2 & 3 & 4 & 5 & 6 & 7 & 8 & 9 & 10 & 11 & 12\end{array}$

Figure 4.15 SLP65 binds preferentially to small liposomes.

A) Size distribution of SUVs and LUVs, both consisting of $75 \%$ PC and $25 \%$ PE, and prepared by detergent removal and extrusion methods, respectively, was measured by DLS. B) The liposome floatation assay was performed with $\mathrm{His}_{6}$-tagged SLP65 WT and PC:PE SUVs/LUVs. The liposome concentration was adjusted prior to incubation with the protein based on the absorbance of TexasRed at $590 \mathrm{~nm}$. Representative immunoblots, developed with an a-SLP65 antibody, are shown. C) The quantification of band intensities in the uppermost two fractions (bound protein) vs. lowermost four fractions (free protein) is shown. $n=3 \pm S D$. D) The liposome floatation experiment was performed with recombinant Hsv2, kindly provided by A. Scacioc, and 5\% PI3P-containing SUVs/LUVs, in HP150 buffer, pH 7.4. The floatation experiment, employing liposomes of the same lipid composition, but prepared in LEW buffer, was performed with SLP65. Representative immunoblots, developed with $\alpha-S L P 65$ and $\alpha-H s v 2$ antibodies, are shown. 
Preferential binding of SLP65 to SUVs indicated that it can recognize membrane curvature. To test this hypothesis in vivo, I generated chimeric proteins where the $\mathrm{N}$ terminal region of SLP65 was substituted with known curvature sensitive lipid-binding domains. I chose two well-characterized curvature-recognition domains, the N-BAR (BinAmphiphysin-Rvs) domain of Amphyphisin and the ALPS (Amphipatic Lipid Packing Sensor) motif of $\alpha$-Synuclein. BAR domains are composed of oligomerized long a-helices which bind to curved membranes due to their crescent-shape. ALPS motifs are unfolded hydrophobic sequences, which adhere weakly to loosely packed membranes and fold into amphipatic a-helices on the membrane surface (reviewed by Antonny, 2011). I cloned the cDNA for the mouse Amphiphysin BAR domain and the a-Synuclein ALPS motif and generated the chimeric constructs N-BAR- $\triangle N$ SLP65 and ALPS- $\triangle N$ SLP65, shown in Figure $4.16 \mathrm{~A}$. These chimeras were expressed in DT40 s/p65 cells and assessed for their ability to reconstitute $\mathrm{Ca}^{2+}$ signaling.

As shown in Figure $4.16 \mathrm{~B}$, the chimeric protein harboring the N-BAR domain of Amphiphysin (N-BAR- $\Delta N$ ) could fully support BCR-induced $\mathrm{Ca}^{2+}$ mobilization. It was also distributed similarly to WT SLP65 - it localized in the intracellular dot-like structures in resting cells and at the plasma membrane in BCR-stimulated cells (Figure 4.16 C). Moreover, this chimeric protein was efficiently tyrosine-phosphorylated upon BCR stimulation as shown in Figure 4.16 D (lanes 6-9). The ALPS domain of a-synuclein, on the contrary, did not target SLP65 to intracellular vesicles, nor could it bring the protein to the plasma membrane (Figure $4.16 \mathrm{C}$ ). Therefore it is not surprising that the ALPS- $\Delta N$ chimera had a weak $\mathrm{Ca}^{2+}$ mobilization profile, similar to that of SLP65 $\Delta \mathrm{N}$ (Figure 4.16 B). 
A)

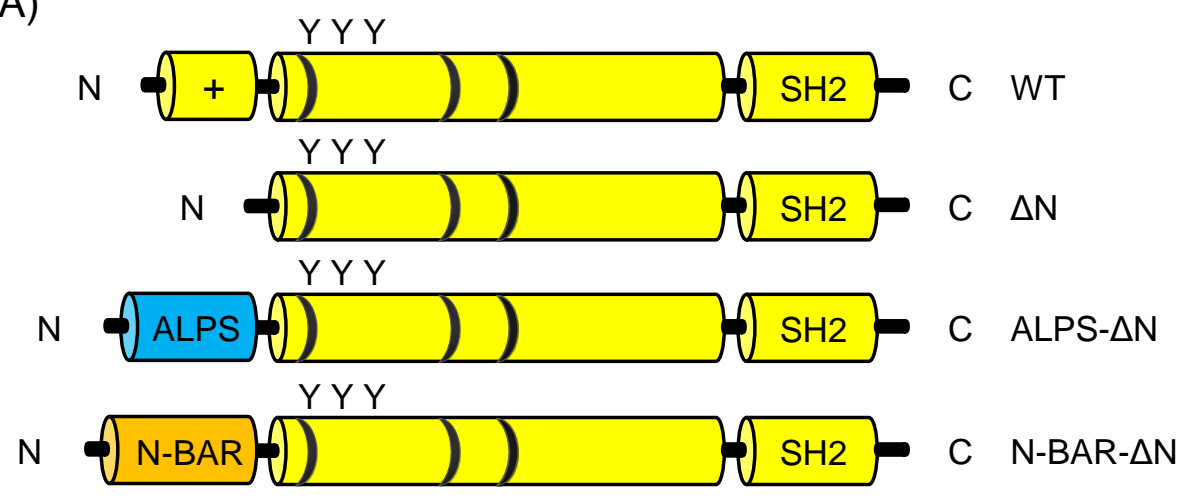

B)

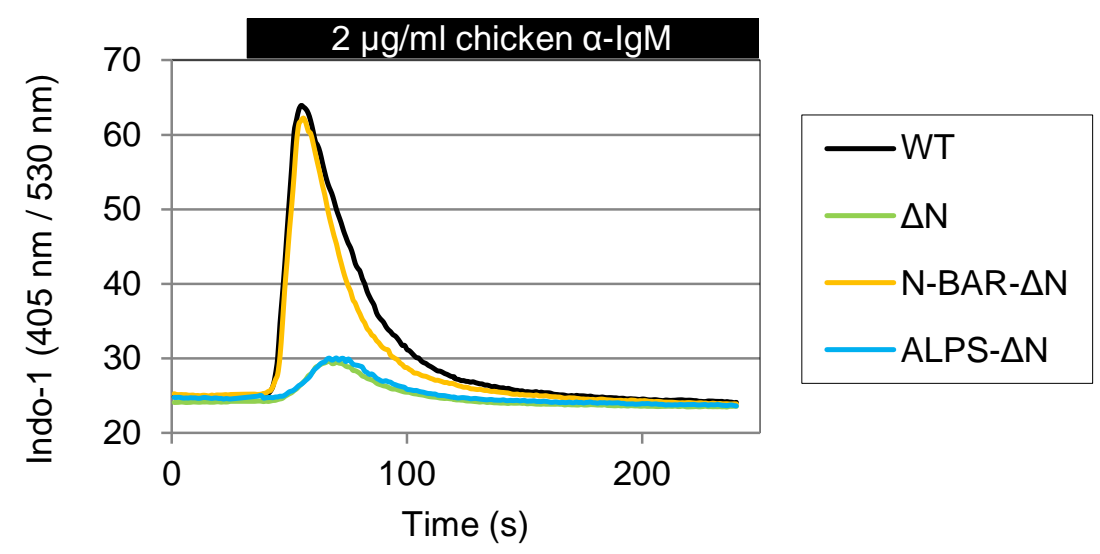

C)

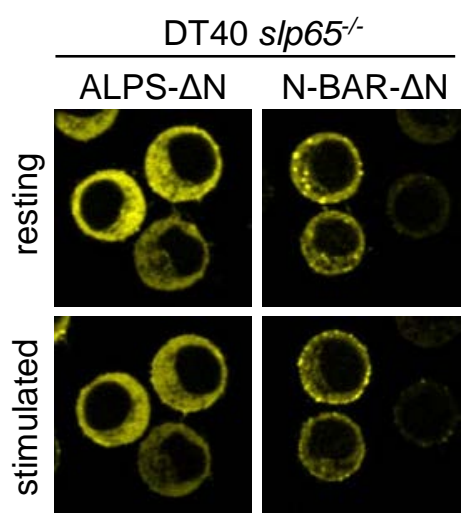

D)

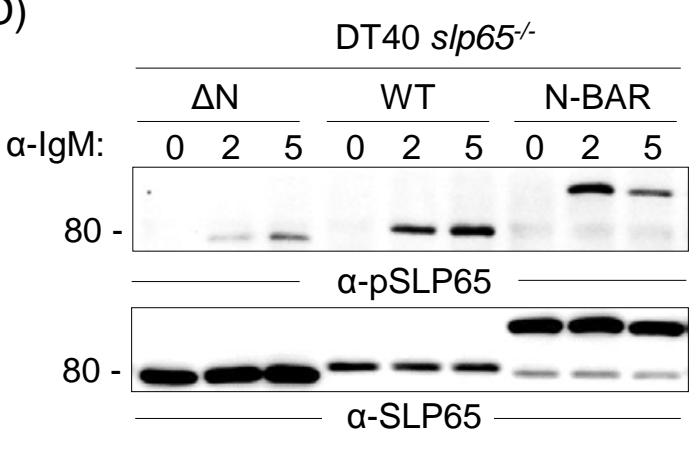

$\begin{array}{lllllllll}1 & 2 & 3 & 4 & 5 & 6 & 7 & 8 & 9\end{array}$

Figure 4.16 The $\mathrm{N}$ terminus of SLP65 can be substituted by the N-BAR domain of Amphiphysin.

A) Schematic representation of the chimeric SLP65 variants containing curvature-sensing lipidbinding domains. B) The constructs represented in A were retrovirally transduced into s/p65 $5^{-1}$ DT40 cells. $\mathrm{Ca}^{2+}$ mobilization was analyzed by flow cytometry. The $\mathrm{Ca}^{2+}$ flux profiles of cells with equal Citrine expression are shown. C) CLSM imaging analysis of Citrine-tagged SLP65 N-BAR and ALPS variants. Images taken before and $3 \mathrm{~min}$ after stimulation with $2 \mu \mathrm{g} / \mathrm{ml} \alpha$-chicken IgM are shown. D) CCLs were prepared from resting and BCR-stimulated cells expressing SLP65 WT, NBAR- $\Delta N$ or $\Delta N$, and an immunoblot was performed with an a-phospho-SLP65 antibody. 
I showed that curvature-sensitive N-BAR domain of Amphiphysin can substitute the SLP65 $\mathrm{N}$ terminus and support BCR signaling. This implies that this domain targeted SLP65 to vesicles similar to those recognized by the endogenous SLP65. To confirm that the N-BAR-SLP65 chimera was indeed localized similarly to WT SLP65, I compared the degrees of colocalization of WT and N-BAR- $\triangle N$ SLP65 with quinacrine. Both variants colocalized equally well with this marker, indicating that similar types of vesicles were targeted by the N-BAR- $\triangle$ N SLP65 fusion protein (Figure 4.17).
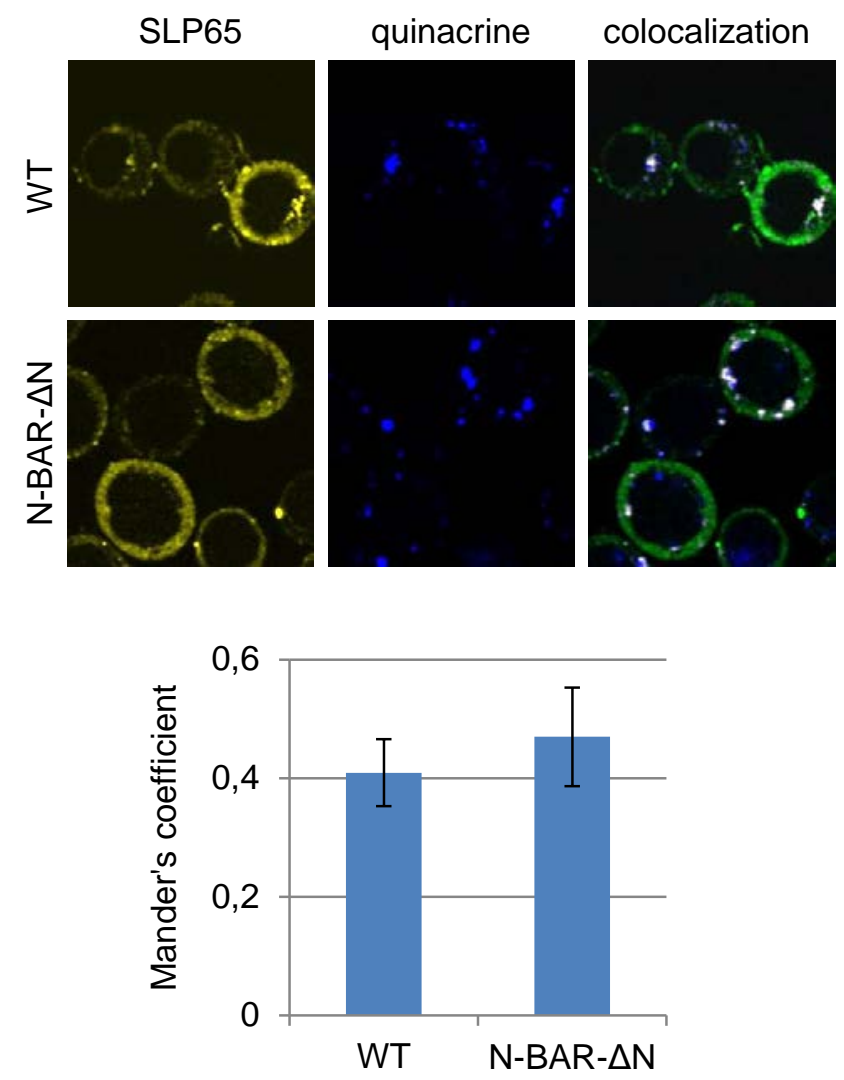

Figure 4.17 N-BAR- $\triangle$ N SLP65 colocalizes with quinacrine-positive vesicles.

DT40 s/p65 $5^{--}$cells reconstituted with WT or N-BAR- $\triangle N$ SLP65 were stained with quinacrine. Zstacks of images in the Citrine- and Cerulean- channel were taken and colocalization of SLP65 and quinacrine was analyzed by Image J. For quantification, the means \pm SD of 10 Z-stacks (each containing $\sim 10$ cells) are shown. 
The data presented so far showed that anchoring SLP65 to small vesicles via the N-BAR domain of Amphiphysin suffices for signaling. It has been reported, however, that SLP65CIN85 interaction also contributes to the precise subcellular localization of SLP65. I therefore wondered whether the N-BAR- $\triangle N$ SLP65 chimera was still dependent on CIN85 binding or if the curvature-sensing BAR domain could bypass the requirement for CIN85. Proline-rich regions of SLP65 act as CIN85-binding sites, and single point mutations of R to $A$ at 2 of these proline-rich regions suffice to abolish CIN85 binding (Oellerich et al., 2011). I introduced these inactivating mutations to the N-BAR chimeric protein, thereby generating a chimera termed N-BAR- $\triangle N$ SLP65 M23 (Figure $4.18 \mathrm{~A}$ ). This chimeric protein with inactivated CIN85-binding sites had a strongly compromised $\mathrm{Ca}^{2+}$ mobilization profile and was not recruited to the plasma membrane after BCR-stimulation. Moreover, it was localized exclusively in the cytosol of resting cells, and not in the vesicular structures (Figure $4.18 \mathrm{~B}$ ). Additionally, no vesicular localization of full-length SLP65 harboring the M23 mutations was observed (Engelke et al., 2014). This result indicated that the membrane-binding by the $\mathrm{N}$ terminus alone is insufficient for vesicular targeting of SLP65. Instead, CIN85 binding co-operates with the SLP65 N terminus for bringing SLP65 to its precise subcellular compartment. In addition, CIN85 binding could also amplify the signaling capability of vesicle-associated SLP65, since CIN85 is able to dimerize via its CC domain (Bremes, 2012 and unpublished data from the lab) and could therefore nucleate the formation of larger SLP65-containing signaling complexes at vesicular membranes. 
A)

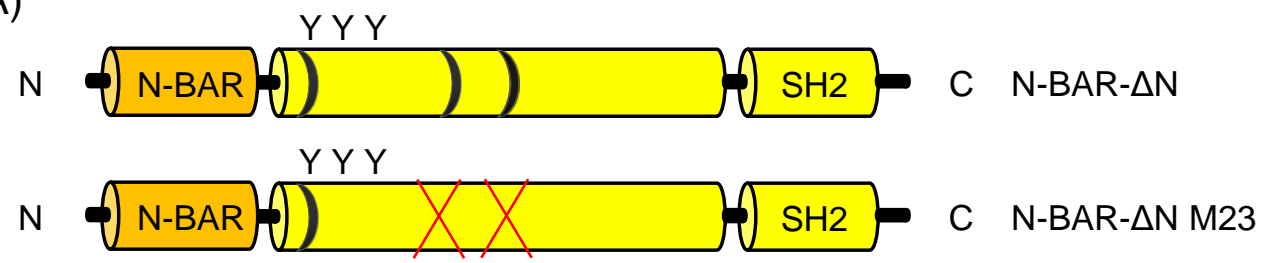

B)

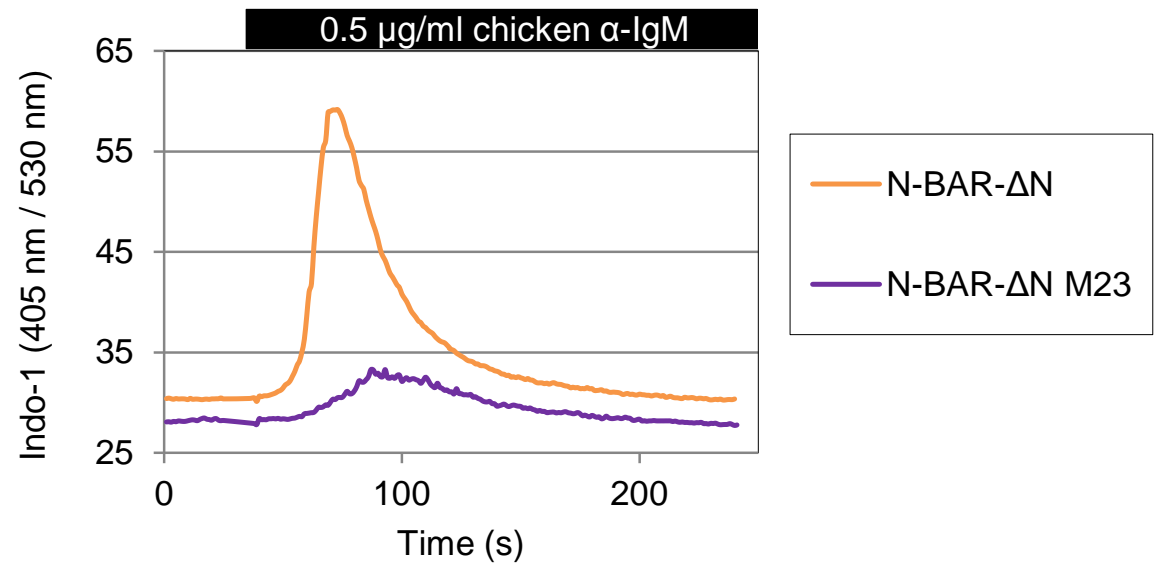

C)

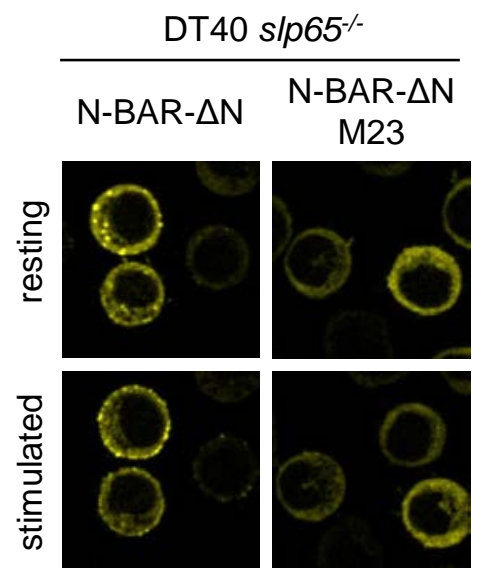

Figure 4.18 N-BAR- $\Delta \mathrm{N}$ chimera is dependent on CIN85 binding.

A) Schematic representation of the chimeric proteins harboring the N-BAR domain at the $N$ terminus fused to either SLP65 $\triangle N$ WT or to SLP65 $\triangle N$ M23 (harboring the inactivating mutations R248,331A. B) DT40 s/p65 ${ }^{-/}$cells were retrovirally transduced with the $\mathrm{N}$-terminally Citrine-tagged constructs represented in (A) and $\mathrm{Ca}^{2+}$ flux was monitored by flow cytometry. The $\mathrm{Ca}^{2+}$ profiles of the cells with equal Citrine expression are shown. C) CLSM imaging analysis of the cells expressing Citrine-tagged N-BAR- $\Delta N$ and N-BAR- $\Delta N$ M23 chimeras. Images before and 3 min after stimulation with $2 \mu \mathrm{g} / \mathrm{ml} \alpha$-chicken $\mathrm{M} 4$ antibody are shown. 


\subsubsection{The CC domain of CIN85 is a lipid-binding domain, which preferentially binds to phosphatidic acid}

My data indicated that CIN85 binding and the N terminus of SLP65 act in concert for proper membrane targeting of SLP65. Data accumulated by J. Kühn has shown that CIN85 mainly mediates its SLP65 targeting-related function via the CC domain (unpublished data). Interestingly, the CIN85 CC domain was also reported to have a lipidbinding function, similarly to the $\mathrm{N}$ terminus of SLP65. The CC domain was shown to bind phosphatidic acid (PA), as detected by lipid-protein overlay PIP-strip assays (Zhang et al., 2009). I aimed to confirm the binding of CIN85 to PA using the liposome floatation assays.

SUVs containing 1,5 or $10 \%$ PA were prepared with the "detergent removal" method. The recombinant protein harbouring the CIN85 CC domain fused to SLP65 $\Delta N(C C-\Delta N)$ was used in the floatation experiments. SLP65 $\Delta \mathrm{N}$ was used as a control, in order to exclude unspecific binding to PA-containing liposomes. As shown in Figure 4.19, CC- $\Delta \mathrm{N}$ associated with liposomes containing 5 and $10 \%$ PA (left panel), while $\Delta \mathrm{N}$ was mostly found in the bottom fractions, indicating that it did not bind to the liposomes (right panel).

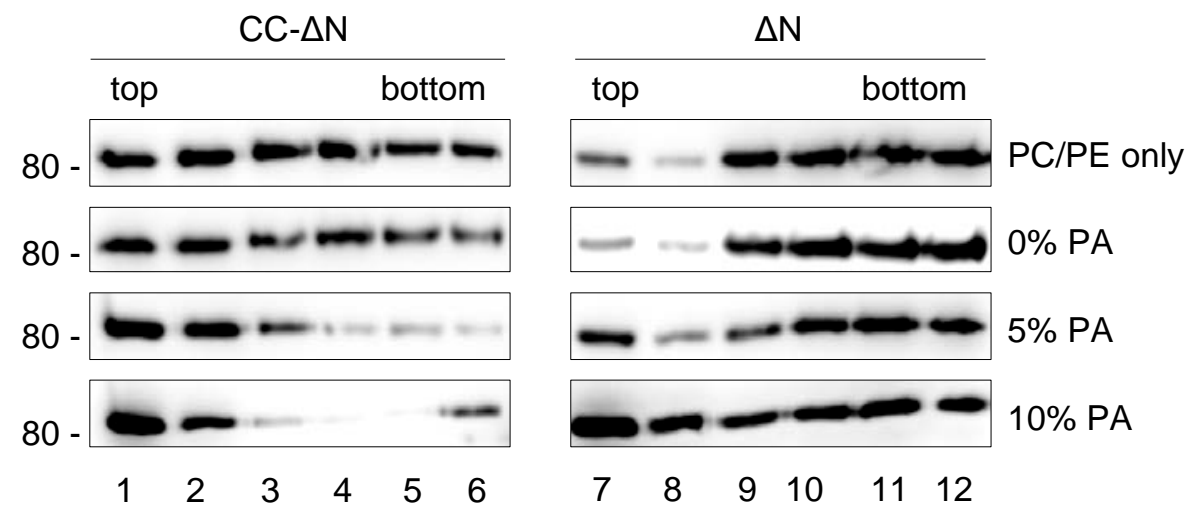

Figure 4.19 CIN85 CC domain binds to PA-containing liposomes.

SUVs, consisting of $75 \%$ PC, 25\% PE (PC:PE only), or containing 1, 5 or $10 \%$ PA (added at the expense of PC) were incubated with recombinant $\mathrm{His}_{6}$-tagged CC- $\Delta N$ or $\triangle N$ SLP65 and a liposome floatation experiment was performed. Representative immunoblots, developed with an $\alpha$-SLP65 antibody, are shown. 
These data show that the CIN85 CC domain can bind PA in vitro. It is possible that this interaction targets CIN85 to vesicular membranes inside the cell. CIN85 could then recruit SLP65 to PA-positive vesicles, thereby enriching SLP65 at specific vesicular membranes. It is however not clear if the affinity of the CC domain for PA is sufficiently high to recognize this relatively low abundant lipid inside the cell. The functional role of CIN85-PA interaction, as well as further properties of the CIN85 CC domain was investigated by $\mathrm{J}$. Kühn during his doctoral work.

\subsubsection{The SLP65 $\mathrm{N}$ terminus is disordered in solution, but binds to liposomes in a defined manner}

The data presented so far indicated that the SLP65 $\mathrm{N}$ terminus is a curvature sensing lipid-binding domain which targets SLP65 to vesicles. In order to better understand how it interacts with membranes, I and my collaborators in the group of Prof. Christian Griesinger aimed at elucidating the conformation and the secondary structure of the $\mathrm{N}$ terminus within the lipid bilayer. For this purpose, recombinant ${ }^{15} \mathrm{~N}-{ }^{1} \mathrm{H}$ labeled protein encompassing the residues 5-50 of SLP65 was expressed in and purified from E.coli. The NMR spectrum of the isolated SLP65 $N$ terminus was recorded and the residue assignment was performed. The low chemical shift dispersion of the backbone amides indicated disordered conformation of the $\mathrm{N}$ terminus in aqueous solution (Figure 4.20 A). This was in accordance with the fast water-amide proton exchange rates, indicating solvent exposure and confirming a lack of secondary structure (data not shown).

Furthermore, I analyzed the secondary structure of the SLP65 N terminus with Circular Dichroism (CD) spectroscopy. $C D$ is a useful tool to estimate protein secondary structure and folding properties, even though it doesn't provide the residue-specific resolution obtained by NMR (Greenfield, 2006). As shown in Figure 4.20 B, the CD spectrum of SLP65 5 -50 closely resembled that of a random coil, with a characteristic minimum of the CD curve around $200 \mathrm{~nm}$. Therefore, it appeared that the SLP65 N terminus, similarly to the large central part of SLP65, lacked secondary structure in solution and had a conformation of an intrinsically disordered protein. This was inconsistent with the prediction by protein secondary structure prediction tools, which predict presence of an amphipathic $\alpha$-helix in the SLP65 $\mathrm{N}$ terminus (prediction by Amphipaseek;(Sapay et al., 2006). I hypothesized that binding to membranes could induce folding of the $\mathrm{N}$ terminus into an a-helical confirmation and therefore investigated the secondary structure of the isolated SLP65 $\mathrm{N}$ terminus in the presence of liposomes. 
A)

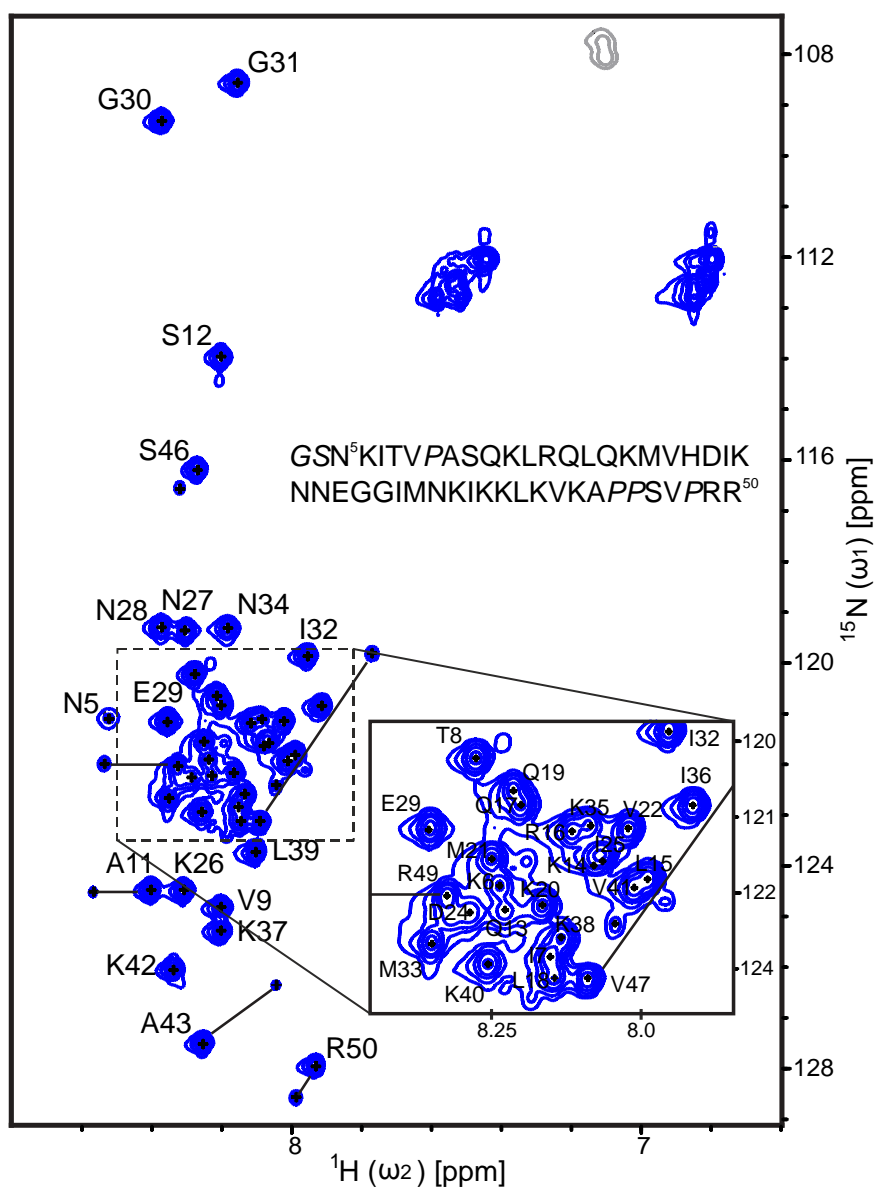

B)

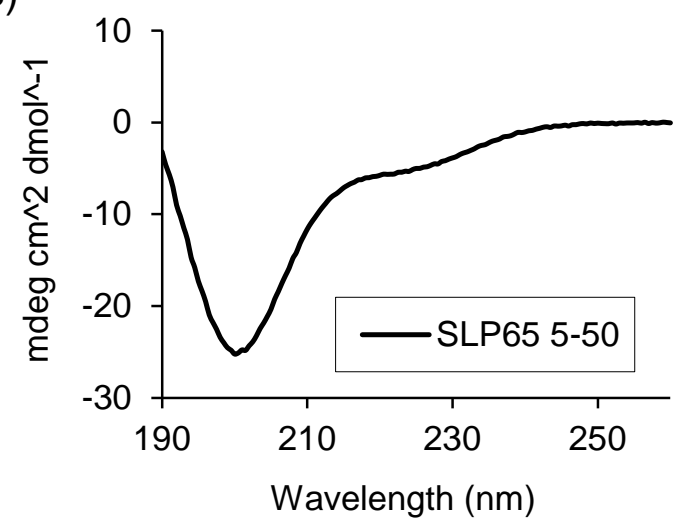

C)

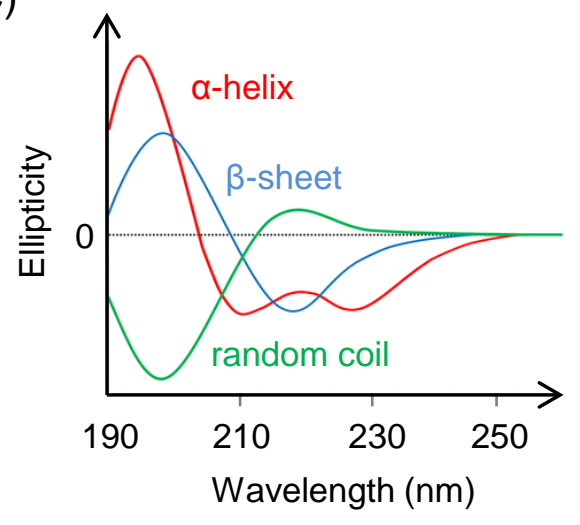

Figure 4.20 The $\mathrm{N}$ terminus of SLP65 is intrinsically disordered in solution.

A) The ${ }^{15} \mathrm{~N}-{ }^{1} \mathrm{H}$ HSQC spectrum of SLP65 $55_{50}$ at $25{ }^{\circ} \mathrm{C}$ in aqueous solution. B) The CD spectrum of SLP65 5 -50 in aqueous solution. C) Reference CD spectra of representative proteins, (image taken from Trivedi \& Chaudhary, http://nptel.ac.in/courses/102103044/module2/lec9/2.html). SLP65550 was cloned and purified by C. Schwiegk, the NMR spectrum was recorded and analyzed by Leo Wong. 
SLP655-50 was tested for binding to SUVs by means of liposome floatation assays. Histagged SLP65 5 -50, harboring a C-terminal ZZ solubility-tag, was incubated with SUVs consisting of $\mathrm{PC}$ and $\mathrm{PE}$ and subjected to the liposome floatation assay. As shown in Figure 4.21 A, the isolated $\mathrm{N}$ terminus was found in the upper liposomal fractions, similarly to WT SLP65, indicating its binding to the liposomes. After confirming that SLP655-50 binds lipids, we proceeded with the structural investigations: the protein was incubated with increasing amounts of SUVs and its NMR and CD spectra were recorded.

Figure 4.21 B shows the NMR spectra of SLP655-50 before and after incubation with SUVs. Upon addition of SUVS, attenuation of intensities of individual peaks on the HSQC spectrum was observed. Adding more concentrated liposome solutions resulted in greater intensity attenuations. The ratio of peak amplitudes after and before the addition of liposomes $\left(A / A_{0}\right)$ was plotted against the amino acid sequence of the protein (Figure 4.21 C). The residues with the highest intensity attenuation (lowest $A / A_{0}$ ) are the ones involved in binding to the liposomes or found in the vicinity of the liposome-binding sites.

The NMR analysis has confirmed the interaction of SLP65 with lipids and identified the individual residues mediating the interaction. However, it was yet not clear whether SLP65 adopts a different secondary structure when bound to the membrane. To further investigate the effect of lipid binding on SLP65 conformation, I recorded the CD spectrum of the $\mathrm{N}$ terminus in the presence of different amounts of SUVs. As can be seen in Figure 4.22 A, addition of PC:PE SUVs did not have an effect on the CD spectrum. However, the $\mathrm{CD}$ spectrum of a protein is derived from the average absorbance of all molecules in the sample. Therefore, if the majority of molecules have one conformation and only a small percentage of molecules have a different conformation, the effect of the low-populated conformation might not be reflected in the CD curve. Given the low affinity of SLP65 for neutral SUVs, it is probable that only few SLP65 molecules are bound to the liposomes. However, even if they are undergoing a conformational change, this would not produce a detectable effect in the $C D$ spectrum. In order to increase the liposome-bound population of SLP65, I recorded the CD spectrum of SLP65 $5-50$ in the presence of negatively charged (PS-containing) SUVs, for which the peptide has a higher affinity. However, as shown in Figure $4.22 \mathrm{~B}$, even in the presence of negatively charged SUVs no change in the CD spectrum was observed, indicating that the random coil conformation of SLP65 persists upon binding to the membrane. 
A)

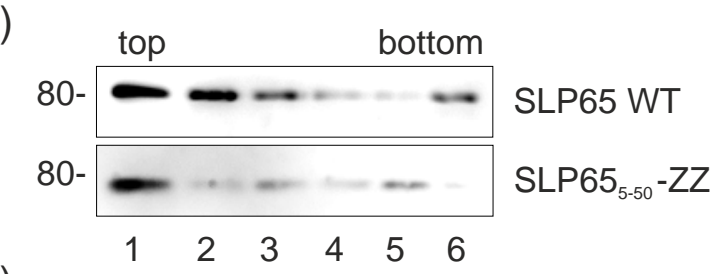

B)

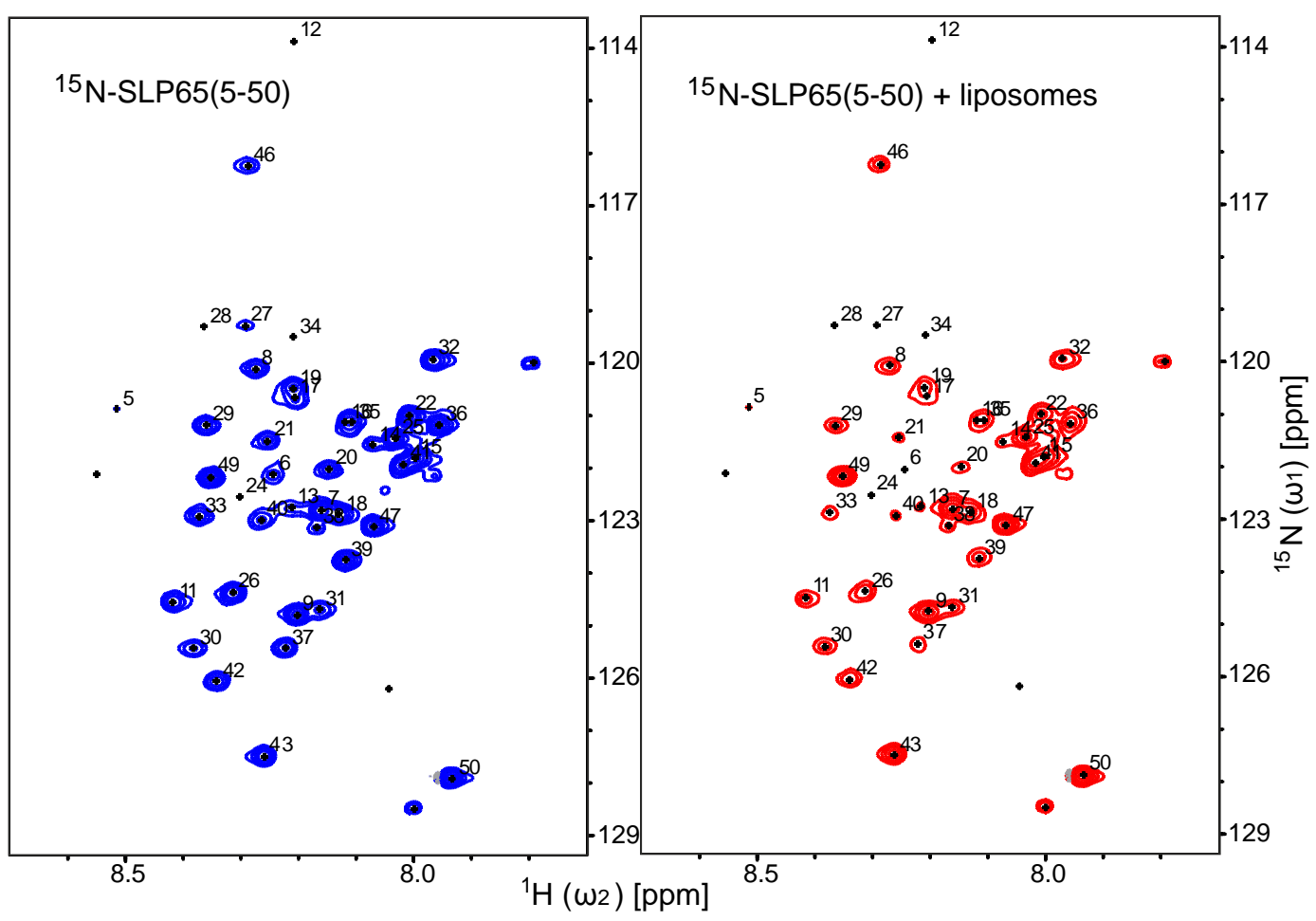

C)

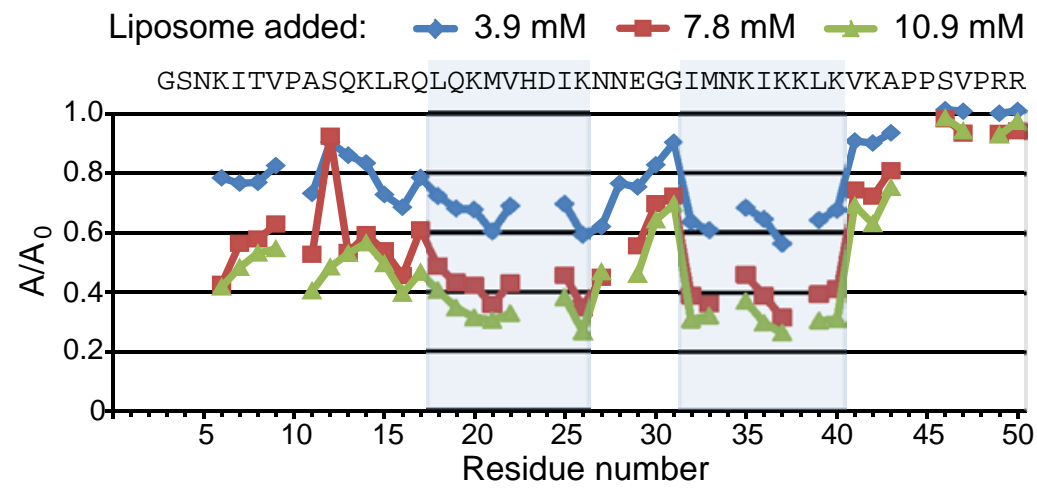

Figure 4.21 SLP65 5 -50 binds to SUVs in a defined manner.

A) A liposome floatation experiment was done with WT SLP65 and SLP655-50. A representative immunoblot, developed with an $\alpha$-His antibody, is shown. B) ${ }^{15} \mathrm{~N}-{ }^{1} \mathrm{H}$ HSQC spectra of SLP65 before and after addition of $10.9 \mathrm{mM}$ (total lipid concentration) liposomes. C) The ratio of peak intensities in HSQC of SLP655-50 before and after addition of liposomes $\left(A / A_{0}\right)$ was plotted against the amino acid sequence of SLP65 $\mathrm{N}$ terminus. The NMR spectra were recorded and analyzed by Leo Wong. 

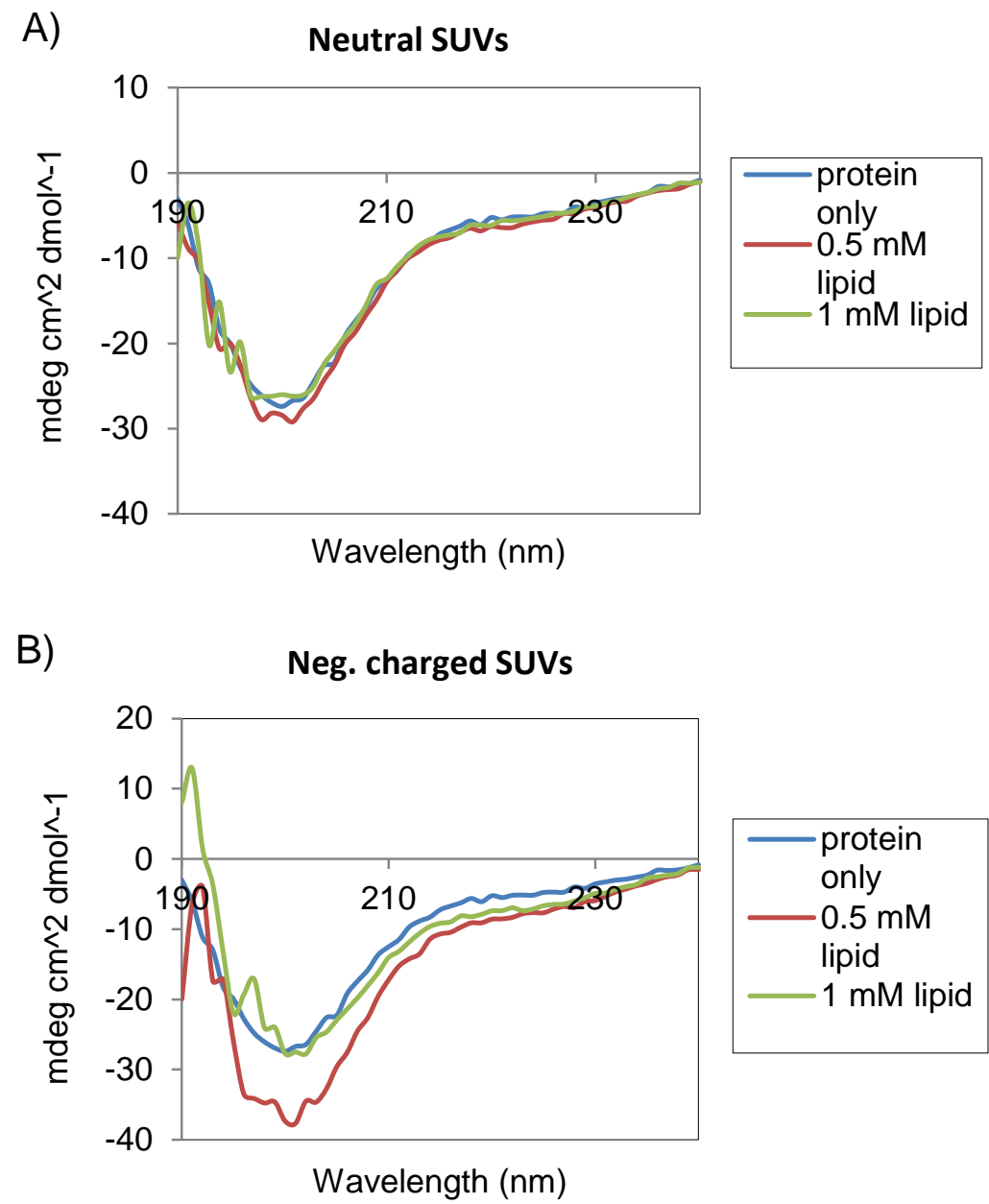

Figure 4.22 Lipid binding does not induce a structural change in the SLP65 N terminus.

CD spectra of SLP655-50 alone or in the presence of SUVs consisting either of (A) PC:PE (75:25) or (B) PC:PE:PS (65:25:10) were recorded. The total lipid concentration of the liposomes was $0.5,1$ or $2 \mathrm{mM}$. However, the spectra with $2 \mathrm{mM}$ lipid had to be discarded due to a high absorbance and a low signal to noise ratio below $200 \mathrm{~nm}$.

The structural analysis of the isolated SLP65 $\mathrm{N}$ terminus demonstrated that it is intrinsically disordered in solution and when bound to vesicles. It still cannot be excluded that lipid binding induces folding of the N terminus of SLP65 under conditions other than those used in our investigations. The NMR and CD experiments were performed at a relatively low protein:lipid ratio, where complete binding of SLP65 to the liposomes might not have been achieved. Therefore the small structural changes may have escaped detection. Moreover, it is not clear whether the $\mathrm{N}$ terminus maintains the disordered conformation in the context of the full length protein. To answer this question, further NMR experiments employing full length SLP65 should be performed. 


\subsubsection{Individual hydrophobic residues in the $\mathbf{N}$ terminus are essential for SLP65 function, but dispensable for lipid binding}

NMR analysis has identified the residues in the SLP65 $\mathrm{N}$ terminus mediating the interaction with lipids. These turned out to be hydrophobic residues, such as leucine, isoleucine, valine and methionine, as well as charged residues, such as lysine. Detailed mutational analysis of the positively charged amino acids in the SLP65 $\mathrm{N}$ terminus has already been performed by N. Hermann in our lab (Herrmann, 2009). She found that substituting individual lysine residues with alanine did not affect the signaling capabilities of SLP65. I proceeded with the genetic analysis of this region of SLP65 and mutated the hydrophobic residues into alanine or glycine. These amino acid substitutions were chosen because alanine is an aliphatic amino acid with a short side chain, making insertion into the membrane unlikely, while glycine has a low propensity to form an a-helix. The following mutants of SLP65 were generated and transduced into s/p65 ${ }^{-1}$ DT40 cells: M21A, M21G, V22A, V22G, I32G, M33A, M33G, I36A, L39A and L39G. The expression level of different variants was confirmed by a Western blot with an a-SLP65 antibody and was comparable (Figure $4.23 \mathrm{C}$ ). In order to assess the signaling capability of the single point mutants of SLP65, $\mathrm{Ca}^{2+}$ mobilization was used as a read out. As shown in Figure 4.23 A, all mutants had compromised $\mathrm{Ca}^{2+}$ mobilization profiles. For simplicity, only alanine mutants are presented here. Substitution to glycine had a very similar effect for most of the residues.

CLSM imaging analysis was performed in order to analyze the subcellular localization and plasma membrane recruitment of the SLP65 variants. As shown in Figure $4.23 \mathrm{~B}$, all mutants had a different subcellular localization from WT SLP65 - the dot-like vesicular distribution was not present and diffuse cytosolic distribution was observed instead. Moreover, many of the mutants, especially M33A/G, I36A and L39A/G, showed nuclear localization in resting and stimulated cells. Finally, some of the mutants were tested for lipid binding via liposome floatation assays. Surprisingly, all of the mutants were able to bind neutral SUVs. The only single point mutation that abolished lipid binding was the L18 to $\mathrm{K}$ substitution. Interestingly, substitution of L18 to A also did not affect SUVs binding. A possible explanation could be that the mutations to A or G, preserving the aliphatic nature of the region, do not interfere with lipid binding of SLP65, whereas a mutation to $\mathrm{K}$ results in a change in polarity and therefore has a disruptive effect on the lipid-binding site. 
A)

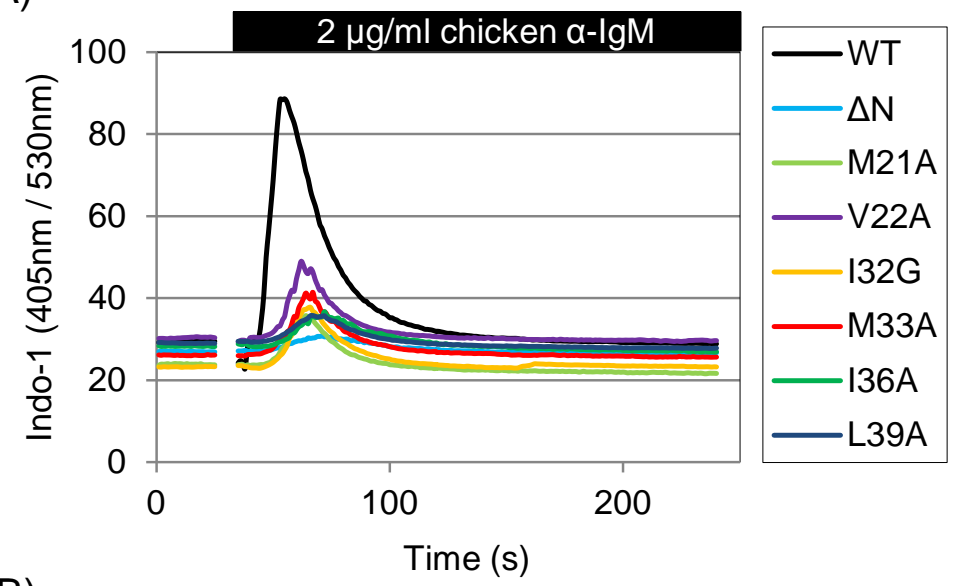

B)

DT40 s/p65-/-

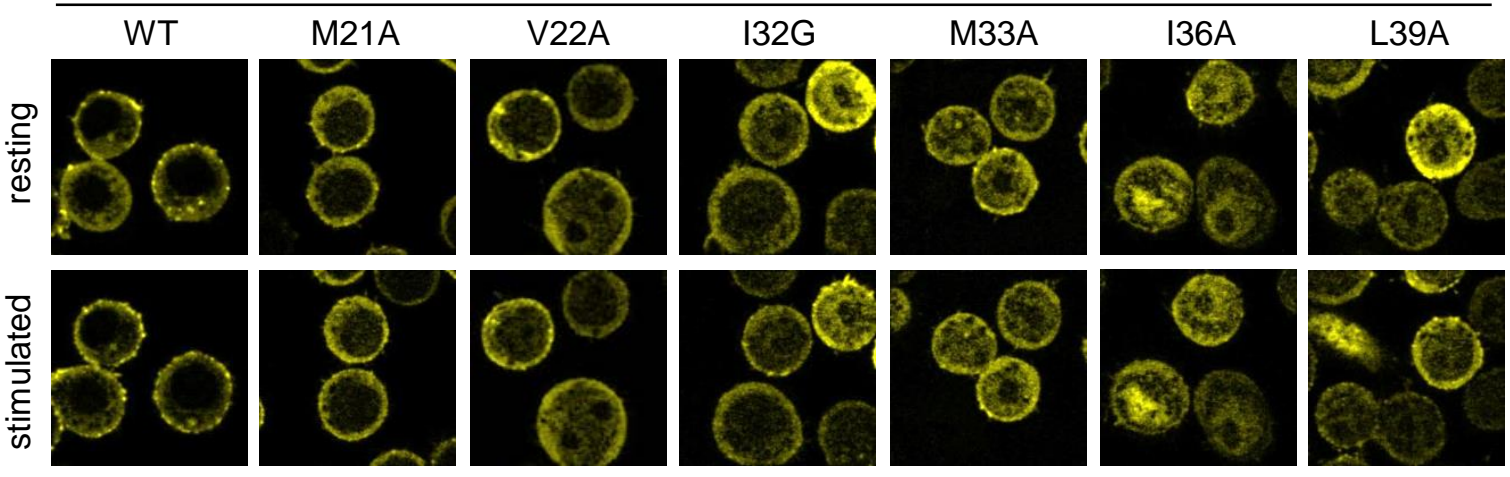

C)

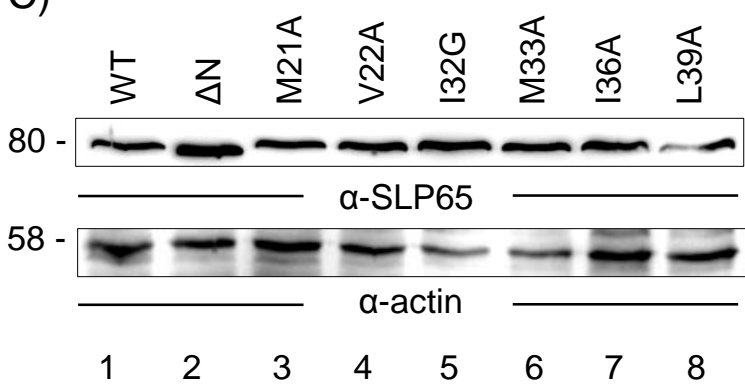

D)

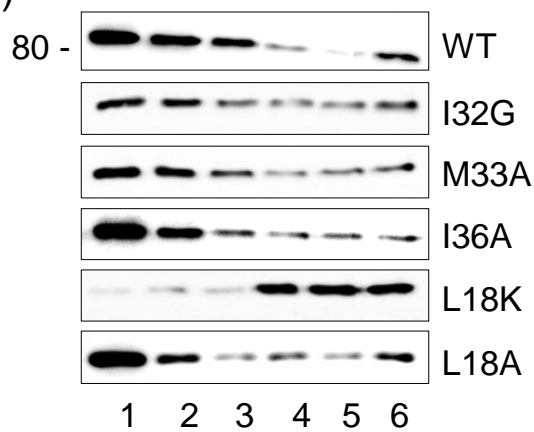

Figure 4.23 Individual hydrophobic residues in the SLP65 N terminus are important for correct subcellular localization and signaling, but are dispensable for lipid binding.

A) DT40 s/p65 cells were reconstituted with Citrine-tagged SLP65 variants harboring single point mutations in the $\mathrm{N}$-terminal region. $\mathrm{Ca}^{2+}$ mobilization was monitored by flow cytometry. The $\mathrm{Ca}^{2+}$ flux profiles of cells with equal Citrine expression are shown. B) CLSM imaging analysis of the Citrine-tagged SLP65 mutants. Images before and 3 mins after stimulation with $2 \mu \mathrm{g} / \mathrm{ml} \alpha$-chicken IgM antibody are shown. C) CCLs, prepared from DT40 cells expressing SLP65 variants, were analyzed for SLP65 expression by immunoblotting. D) A liposome floatation experiment was performed with recombinant $\mathrm{His}_{6}$-tagged SLP65 variants and PC:PE liposomes. Representative immunoblots, developed with an a-SLP65 antibody are shown. 
Collectively, my data indicates that hydrophobic residues in the SLP65 N terminus are critical for SLP65 activation and membrane recruitment, but are dispensable for lipid binding. This implies that the $\mathrm{N}$ terminus uses another mechanism, in addition to lipid binding, for targeting SLP65 to membranes. This could involve, for example, binding to a membrane protein ligand. However, it is still possible that the mutations analyzed actually reduce the affinity of SLP65 for lipids. This suffices to interfere with membrane targeting in the cell, however, the small change in affinity cannot be detected with the floatation assays.

\subsubsection{The $\mathrm{N}$ terminus plays a differential role for SLP65 membrane targeting and $\mathrm{Ca}^{2+}$ signaling in different species and at different stages of $\mathrm{B}$ cell development}

The data presented until now was generated using the SLP65-deficient DT40 cell line. It is a chicken B cell lymphoma line readily undergoing homologous recombination, which facilitates the generation of knock out cell lines (Winding \& Berchtold, 2001). The DT40 cell line can be classified as a pre-B cell line, since its BCR is still undergoing gene conversion of the light chain (Buerstedde et al., 1990; Kim et al., 1990). Moreover, BCR cross-linking in DT40 cells leads to apoptosis, unlike in more mature B cell types, where it induces proliferation and differentiation. Due to the species- and developmental stagespecific properties of DT40 cells, I aimed to confirm my data on the function of the SLP65 $\mathrm{N}$ terminus in a mature human $\mathrm{B}$ cell line.

I used the SLP65-deficient DG75 cell line, kindly provided by C. Hitzing, generated via the TALEN-mediated method. Two s/p65 $5^{-1-}$ clones, \# 7 and \# 28, were reconstituted with SLP65 WT, $\Delta \mathrm{N}$, or some of the single point mutants. The $\mathrm{Ca}^{2+}$ release profiles upon stimulation with $\alpha$-human $\lg \mathrm{M}$ antibodies of transfected and untransfected cells were recorded. For simplicity, the measurements with only one knock out clone, clone \#7, are shown here. S/p65\% DG75 cells mobilized very little $\mathrm{Ca}^{2+}$ upon BCR-stimulation, even though the $\mathrm{Ca}^{2+}$ flux of the knock out cell line was still stronger than that of the s/p65 ${ }^{-1}$ DT40 cell line (Figure $4.24 \mathrm{~A}$, red line). This could be due to the presence of alternative adaptor proteins, which substitute the function of SLP65 in DG75 cells. Reconstitution of the cells with WT SLP65 resulted in a full restoration of the $\mathrm{Ca}^{2+}$ mobilization profile. Expression of the SLP65 variant lacking the N-terminal region, SLP65 $\Delta N$, led to strongly impaired $\mathrm{Ca}^{2+}$ mobilization. Surprisingly, the single point variant SLP65 L18K, had a robust, only marginally impaired $\mathrm{Ca}^{2+}$ response (Figure $4.24 \mathrm{~A}$ ). This was inconsistent with the data obtained from DT40 cells, where this variant elicited a very weak $\mathrm{Ca}^{2+}$ response, 
comparable to that of SLP65 $\Delta \mathrm{N}$. Even more surprisingly, the ability of SLP65 L18K to support $\mathrm{Ca}^{2+}$ mobilization did not correlate with its ability to translocate to the plasma membrane upon BCR stimulation. The CLSM imaging analysis showed that both SLP65 $\Delta \mathrm{N}$ and L18K were not recruited to the plasma membrane. On the other hand, membrane translocation of WT SLP65 was also weaker and more difficult to detect than in DT40 cells (Figure 4.24 B).

Finally, the $\mathrm{Ca}^{2+}$ mobilization profiles of other single point mutants of SLP65 were normal and comparable to that of WT SLP65 (Figure $4.24 \mathrm{C}$ ). These data indicated that even though the N-terminal region is required for SLP65 function also in DG75 cells, the contribution of individual hydrophobic residues to SLP65 membrane translocation and signaling might be different in human B cells, as compared to the chicken DT40 B cell line. It is also possible that the requirement for the SLP65 $\mathrm{N}$ terminus is higher in the immature DT40 B cell line than in mature DG75 cells.

In order to further investigate the functional role of the SLP65 $\mathrm{N}$ terminus for SLP65 activation and $\mathrm{Ca}^{2+}$ signaling, I performed experiments with s/p65 $65^{-1}$ primary mouse B cells. It has already been reported that the $N$ terminus of SLP65 is required for pre-BCR signaling in mouse B cells. S/p65\% pre-B cells reconstituted with signaling-incompetent variants of SLP65 I25E and L18E were unable to down-regulate their pre-BCR expression and to differentiate toward $\mathrm{K}$ light chain surface expression (Kohler et al., 2005). I wondered whether SLP65 also depends on its N terminus for signaling in mature mouse B cells. I therefore isolated primary naïve B cells from spleens of adult s/p65 mice, stimulated them with LPS to induce proliferation, and reconstituted them with Citrinetagged SLP65 WT, $\Delta N$ or L18K. The improved protocol for transfection of mouse splenocytes, optimized by L. König, was used (Bremes, 2012; König, 2012). The transfection efficiency 2 days after infection was 1-5\%, as shown in Figure $4.25 \mathrm{~A}$. Transfection of s/p65\% cells with all three constructs restored otherwise negligible $\mathrm{Ca}^{2+}$ flux of the knock out cells, even though the splenocytes transfected with WT SLP65 had the strongest $\mathrm{Ca}^{2+}$ response (Figure $4.25 \mathrm{~B}$ ). SLP65 $\Delta \mathrm{N}$ and L18K had only mildly compromised $\mathrm{Ca}^{2+}$ mobilization profiles, indicating that mature mouse $\mathrm{B}$ cells are less dependent on the SLP65 N terminus than mouse pre-B cells or the immature B cell line DT40. Stimulation of the cells with less antibody $(10$ or $5 \mu \mathrm{g} / \mathrm{ml})$ resulted in a slightly lower $\mathrm{Ca}^{2+}$ response, but the small difference between the $\mathrm{Ca}^{2+}$ curves of WT and $\Delta \mathrm{N}$ or L18K variants was preserved (data not shown). 
A)

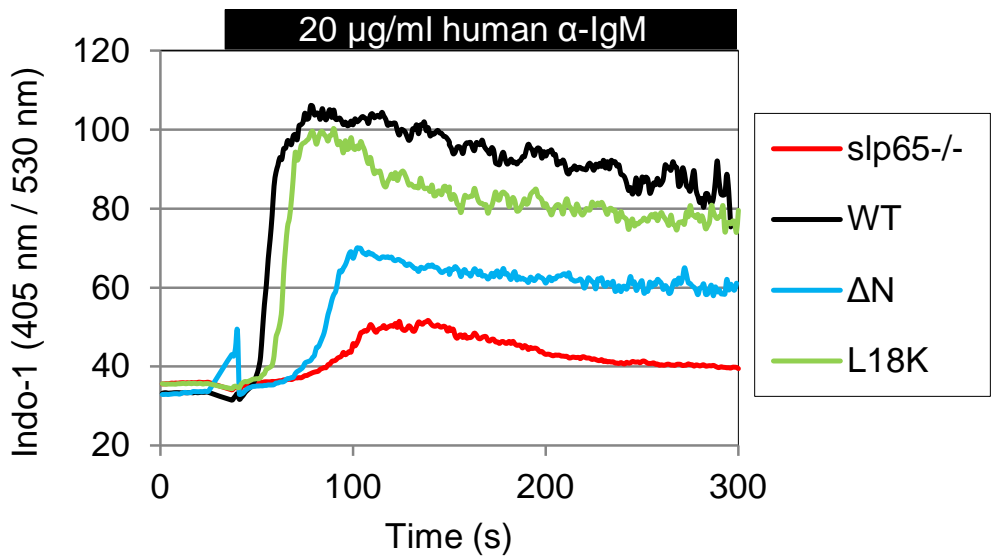

B)

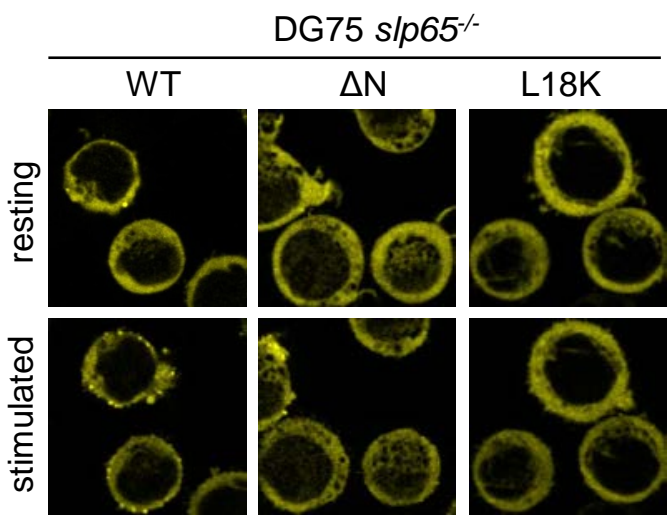

C)

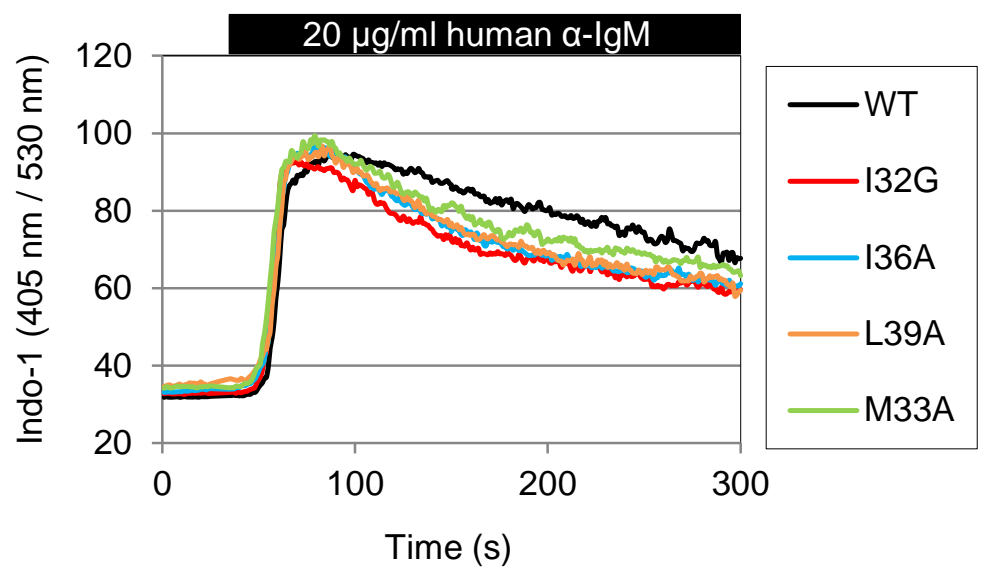

Figure 4.24 The $\mathbf{N}$ terminus of SLP65 is required, but individual hydrophobic residues are dispensable for SLP65 function in DG75 cells.

SIp65 ${ }^{-1}$ DG75 cells, kindly provided by C. Hitzing, were retrovirally transduced with constructs encoding Citrine-tagged SLP65 WT, $\triangle \mathrm{N}$ or the single point mutants L18K, I32G, M33A, I36A or L39A. A) and C) $\mathrm{Ca}^{2+}$ mobilization in the knock out cell line and reconstituted cells was monitored by flow cytometry. $\mathrm{Ca}^{2+}$ mobilization profiles of cells with equal Citrine expression are shown. B) CLSM imaging analysis of the Citrine-tagged SLP65 mutants. Images before and 3 min after stimulation with $20 \mu \mathrm{g} / \mathrm{ml} \mathrm{\alpha -human} \mathrm{IgM} \mathrm{antibody} \mathrm{are} \mathrm{shown.}$ 
A)
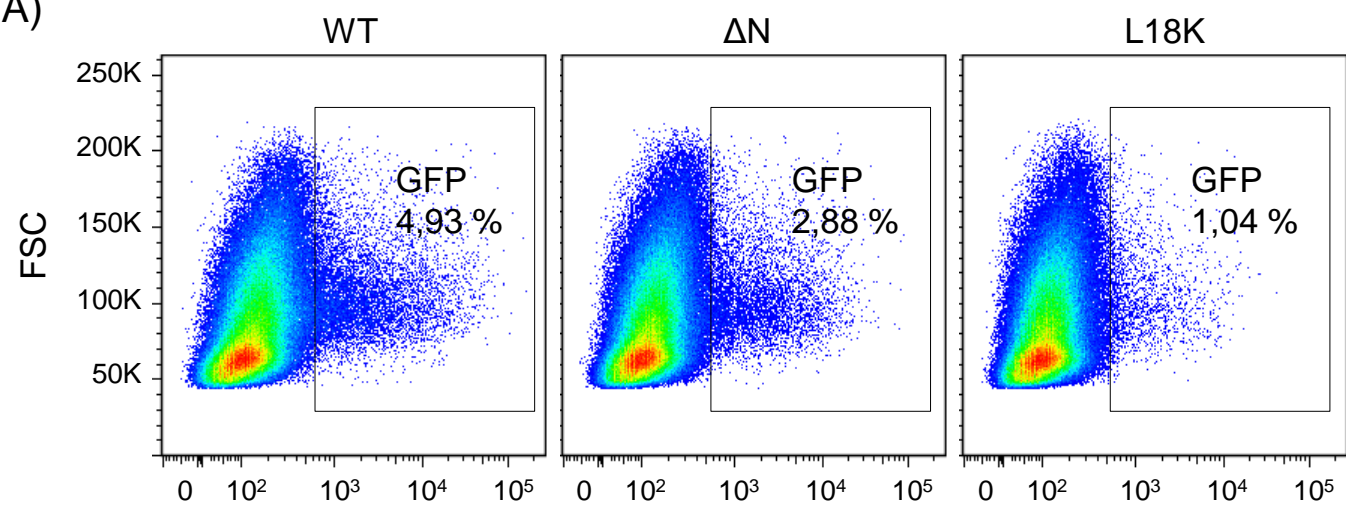

FITC

B)

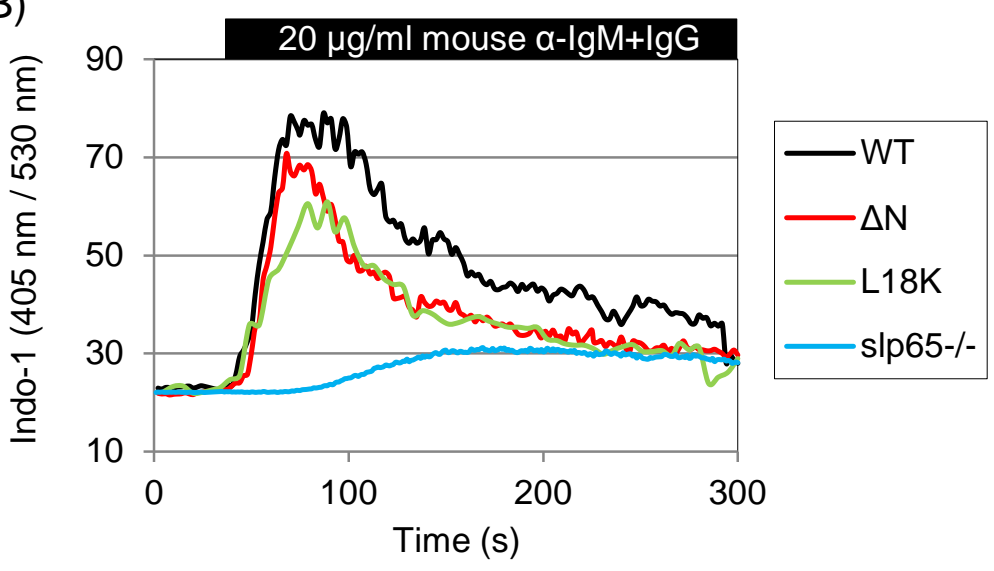

C)

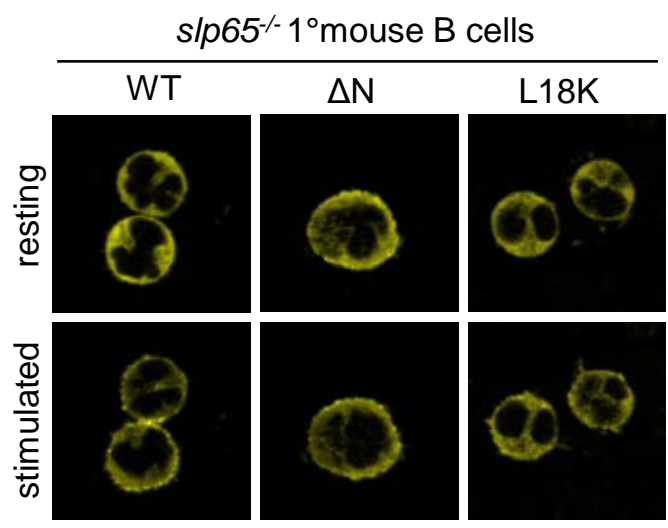

Figure 4.25 The $\mathrm{N}$ terminus of SLP65 is dispensable for $\mathrm{Ca}^{2+}$ signaling in mouse primary $\mathrm{B}$ cells.

Splenocytes isolated from s/p65 $5^{-/}$mice were stimulated with LPS and retrovirally transduced with constructs encoding N-terminally Citrine-tagged SLP65 WT, $\triangle N$ or L18K. A) The transfection efficiency is indicated with a gate on Citrine-positive cells. B) $\mathrm{Ca}^{2+}$ mobilization was analyzed in parental and transfected cells with flow cytometry. The $\mathrm{Ca}^{2+}$ mobilization profiles of cells with equal Citrine-expression are shown. C) CLSM imaging analysis of transfected cells before and 3 min after stimulation with $20 \mu \mathrm{g} / \mathrm{ml} \alpha$-mouse $\lg \mathrm{M}+\lg G$ Fab fragment. 
Finally, subcellular localization and plasma membrane recruitment of SLP65 was analyzed in transfected cells with CLSM. In resting cells, all SLP65 variants localized to the cytosol (Figure 4.25 B). No dot-like vesicular distribution could be observed. However, one should note that due to the low transfection efficiency only a small number of cells could be analyzed. In stimulated cells, the translocation of WT SLP65 to the plasma membrane, but not that of $\Delta \mathrm{N}$ and L18K variants, was observed. This result was consistent with the data from DG75 cells, where in a similar manner, SLP65 recruitment but not $\mathrm{Ca}^{2+}$ mobilization was affected by the mutations or the deletion of the SLP65 N terminus. A possible explanation for this observation is a small number of SLP65 molecules being required to be present at the plasma membrane to initiate $\mathrm{Ca}^{2+}$ mobilization. In this case, even though plasma membrane targeting of N-terminal SLP65 variants is defective and incomplete, enough molecules of SLP65 make it to the plasma membrane to initiate the $\mathrm{Ca}^{2+}$ release. Therefore the differences in the $\mathrm{Ca}^{2+}$ mobilization profiles are small or not observed.

In conclusion, data from the DG75 cell line and mouse primary B cells indicated that the requirement for the SLP65 $\mathrm{N}$ terminus is different depending on the species and the developmental stage. This can be explained by differential expression of binding partners and proteins participating in SLP65 transport in chicken, mouse and human, and at different stages of B cell development.

\subsubsection{The SLP65 $\mathrm{N}$ terminus depends on the remaining SLP65 sequence for full functionality}

The data presented until now has indicated that the SLP65 $\mathrm{N}$ terminus has a previously unrecognized ability to bind lipids, which might target it to vesicles in living cells. Liposome floatation and NMR data have also shown that the isolated $N$ terminus, i.e. the residues 550 of SLP65, can bind liposomes. I next asked the question whether the $\mathrm{N}$ terminus can function as an autonomous protein domain and confer membrane localization to other proteins.

In order to investigate whether the $\mathrm{N}$ terminus could function independently of its original location in SLP65, I generated a construct where the N-terminal 50 amino acids were deleted and newly fused at the $\mathrm{C}$ terminus, i.e. downstream of the SLP65 SH2 domain. This fusion protein, named SLP65 $\Delta \mathrm{N}-\mathrm{Nt}$ (Figure $4.26 \mathrm{~A}$ ), was expressed in s/p65 ${ }^{-1-}$ DT40 cells and its subcellular localization and the ability to support $\mathrm{Ca}^{2+}$ signaling were analyzed. As shown in Figure $4.26 \mathrm{~B}$, the cells expressing the $\Delta \mathrm{N}$-Nt chimeric protein 
mounted a slightly better $\mathrm{Ca}^{2+}$ response than cells expressing the truncated variant SLP65 $\Delta \mathrm{N}$. However, the $\mathrm{Ca}^{2+}$ response of $\Delta \mathrm{N}-\mathrm{Nt}$ was still significantly lower than that of WT SLP65. Consistent with its $\mathrm{Ca}^{2+}$ mobilization profile, SLP65 $\Delta \mathrm{N}-\mathrm{Nt}$ localized predominantly to the cytosol and no plasma membrane recruitment could be observed (Figure 4.26 C). These results indicated that the SLP65 $\mathrm{N}$ terminus at least partially depends on the remaining SLP65 sequence for full functionality. It is however also possible, that in the $\Delta \mathrm{N}$-Nt chimera the newly placed $\mathrm{N}$ terminus is too close to the SH2 domain of SLP65 and interferes with its function. Since the $\mathrm{SH} 2$ domain is indispensable for BCR-targeting of SLP65, this could account for the low $\mathrm{Ca}^{2+}$ mobilization profile of this chimeric protein.

Finally, I wanted to see if the SLP65 $\mathrm{N}$ terminus could confer membrane association to another protein. In the study by Koehler et al, the localization of the SLP65 N terminusGFP fusion protein was investigated in the mouse B lymphoma cell line K46. This fusion protein localized predominantly to the cytosol, however, photo-bleaching of the GFP in the cytosol revealed that some SLP65 Nt-GFP was associated with the plasma membrane (Kohler et al., 2005). I have fused the SLP65 N terminus to CIN85, a protein participating in the same signaling cascade and presumably targeted to similar kind of vesicles as SLP65. CIN85 uses its CC domain for membrane association, since the mutant variant of CIN85 lacking this domain (CIN85 $\Delta C C$ ) is not recruited to the plasma membrane upon BCR ligation (Zhang et al., 2009; Bremes, 2012). I generated a chimeric construct where the CC domain of CIN85 was replaced by the N terminus of SLP65 (Figure 4.26 A). This chimera ( $\triangle \mathrm{CC}-\mathrm{Nt}$ ) was expressed in $\operatorname{cin} 85^{-/-}$DG75 cells, generated and kindly provided by K. Schulz, and its subcellular localization and plasma membrane recruitment was monitored by CLSM. As shown in Figure $4.26 \mathrm{C}$, the CIN85-SLP65 chimeric protein $\triangle \mathrm{CC}$ $\mathrm{Nt}$ was distributed in the cytosol of resting and stimulated cells, similarly to $\triangle \mathrm{CC} C \mathrm{CIN85}$, i.e. no plasma membrane translocation of this variant was observed. In contrast, WT CIN85 was readily recruited to the plasma membrane upon BCR stimulation. These data show that the SLP65 $\mathrm{N}$ terminus cannot provide membrane association to CIN85, implying that the $\mathrm{N}$ terminus is not an autonomous domain and cannot function independently of SLP65. An alternative explanation would be that the affinity of the SLP65 $\mathrm{N}$ terminus for lipids is very low, thereby making membrane association hard to observe with the methods used. 
A)

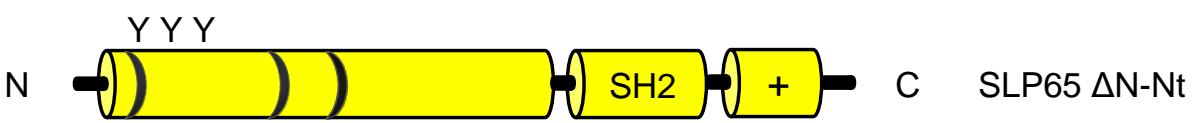

$\mathrm{N}$

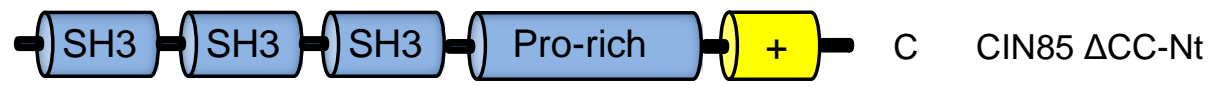

B)

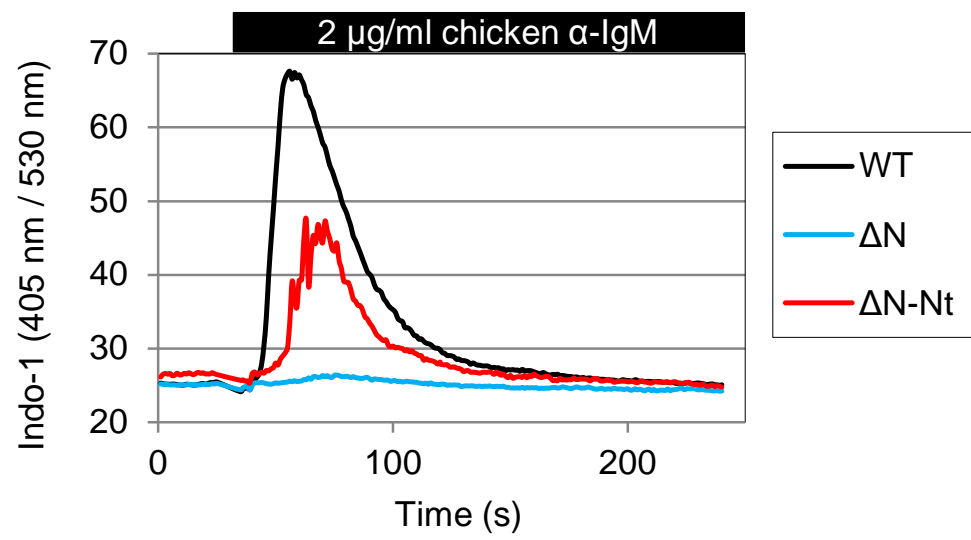

C) $\frac{\mathrm{DT} 40 \mathrm{~s} / \mathrm{p} 65^{-/}}{\Delta \mathrm{N}-\mathrm{Nt}}$

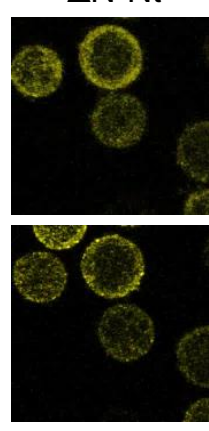

D)

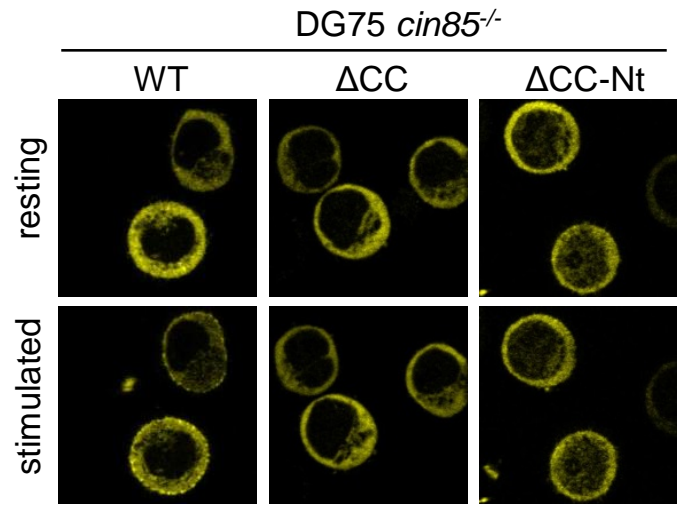

Figure 4.26 The $\mathrm{N}$ terminus cannot function independently of SLP65.

A) Schematic representation of the chimeric constructs SLP65 $\Delta N-N t$ and CIN85 $\Delta$ CC-Nt. B) DT40 s/p65\% cells were reconstituted with SLP65 WT, $\Delta \mathrm{N}$ or $\Delta \mathrm{N}-\mathrm{Nt}$. $\mathrm{Ca}^{2+}$ flux was monitored by flow cytometry. The $\mathrm{Ca}^{2+}$ flux profiles of cells with equal Citrine expression are shown. C) Subcellular localization and plasma membrane recruitment of SLP65 $\triangle \mathrm{N}-\mathrm{Nt}$ was analyzed by CLSM. Images before and $3 \mathrm{~min}$ after stimulation with $2 \mu \mathrm{g} / \mathrm{ml}$ a-chicken IgM antibody are shown. D) DG75 $\operatorname{cin} 85^{-1-}$ cells were retrovirally transduced with Citrine-tagged CIN85 WT, $\triangle \mathrm{CC}$ or $\triangle \mathrm{CC}$-Nt. Subcellular localization and plasma membrane recruitment of CIN85 WT, $\Delta \mathrm{CC}$ and $\Delta \mathrm{CC}$-Nt was analyzed by CLSM. Images before and $3 \mathrm{~min}$ after stimulation with $20 \mu \mathrm{g} / \mathrm{ml}$ a-human IgM antibody are shown. 


\section{Discussion}

B cell activation depends on the assembly of signaling complexes at the plasma membrane, organized by the adaptor protein SLP65. However, the precise mechanism of the plasma membrane targeting of SLP65 was not clear. In my thesis, I focused on deciphering the contribution of the N-terminal region of SLP65 for this process. My colleagues and I found that the $\mathrm{N}$ terminus mediates association of SLP65 with membranes in resting $B$ cells. We showed that the $N$ terminus targets SLP65 to intracellular vesicles in vivo and provides it the ability to bind lipids in vitro. The vesicular association of SLP65 is required for efficient plasma membrane translocation, implying that vesicular transport may deliver SLP65 to the BCR. NMR analysis of the SLP65 N terminus, performed in collaboration with the group of Prof. Christian Griesinger, showed that it is intrinsically disordered in solution, but binds to liposomes in a defined manner. Further structural analysis also identified long disordered regions in the central part of SLP65.

\subsection{Intrinsic disorder in SLP65 and its implications for SLP65 function}

The gap in understanding the activation process of SLP65 was at least partially related to the lack of knowledge about its secondary and tertiary structure. Several attempts to crystallize full-length SLP65 were unsuccessful (unpublished data from the lab), even though the crystal structure of the isolated SH2 domain of mouse SLP65 has been reported (Sano et al., 2007). The difficulties in obtaining a crystal structure can be explained by the presence of large intrinsically disordered regions in SLP65, as revealed by our NMR analysis. This is, at least partially, in agreement with the prediction by the secondary structure prediction softwares, which also predict a lack of secondary structure in the central part of SLP65 preceding the SH2 domain (prediction by the I-TASSER server; Zhang, 2008), but suggest a coiled-coil structure in the $\mathrm{N}$-terminal region (prediction by COILS and Amphipaseek; Lupas et al., 1991; Sapay et al., 2006).

I investigated the functional role of the intrinsic disorder in the central part of SLP65 by rearranging the phosphotyrosine motifs for Btk and PLCY2 binding or by substituting this region with a disordered protein sequence. My results indicate that a secondary structure is not necessary for the assembly of the $\mathrm{Ca}^{2+}$ initiation complex, but might be required for the membrane targeting of SLP65. The intrinsic disorder may facilitate the scaffolding function of SLP65 and enable the simultaneous binding of several proteins to one SLP65 molecule. However, Btk and PLCy2 seem to assemble on SLP65 in a spatially defined 
manner since exchanging their binding sites rendered SLP65 unfunctional (see Figure 4.6). This implies that some degree of structural order should exist also in SLP65, at least in the vicinity of the Btk- and PLCY2-binding sites. It is not uncommon for IDPs to undergo a disorder-to-order transition upon binding their targets (for review, see Dyson \& Wright, 2002). It is therefore possible that Btk and PLCY2 binding promotes SLP65 to take a more ordered conformation. Studies of the SLP65 molecular structure in a complex with Btk and PLCy2 could answer the ultimate question of whether there is an ordered conformation of SLP65 within the $\mathrm{Ca}^{2+}$ initiation complex.

Intrinsically disordered proteins usually bind their targets with high specificity, but low affinity. This is an advantage for signaling proteins that switch between "on" and "off" states for the regulation of signaling cascades. A flexible protein, such as SLP65, can form a more extended interaction surface than a structured protein - the flexibility allows for a precise fit to the target and thereby a high specificity. At the same time, an IDP has a lower affinity for its targets than an ordered protein, because in the latter case a perfectly fitting "bound" conformation exists before the binding takes place (reviewed by Zhou, 2012). Intrinsic disorder in SLP65 could therefore be essential for specific, but reversible binding of Btk and PLCY2, as well as other interaction partners.

My results also indicate that the central part of SLP65 has a previously unrecognized membrane targeting function, in addition to its already known role in membrane anchoring via binding to CIN85. From the cytosolic distribution of the synthetic protein SLP65 GS (see Figure $4.3 \mathrm{C}$ ) it can be assumed that the middle part of SLP65 is also involved in vesicle binding. It might support the function of the SLP65 $\mathrm{N}$ terminus or CIN85 for vesicle targeting or contain a binding site for a vesicle-resident protein. One promising candidate is Unc119, a protein identified as part of the SLP65 interactome, and shown to participate in vesicular trafficking in neurons and T lymphocytes (Gorska et al., 2009; Oellerich et al., 2011; Zhang et al., 2011). Other, yet unidentified, binding partners of SLP65 could also mediate this targeting. However, it is also possible that the GS linker has a dominant negative, i.e. inhibitory effect on one or more of the SLP65 domains important for membrane recruitment. This would explain the phenotype of the GS and CC- $\Delta N$ GS variants (Figure 4.3).

The functional importance of the central part of SLP65 is consistent with the relatively high degree of evolutionary conservation in this region. Not only the proline-rich regions and the tyrosine motifs, but also some negatively charged amino acids are conserved between species. This indicates once again that a total absence of secondary structure is unlikely. 
Disordered sequences are usually less conserved than ordered protein domains. Further functional analysis, e.g. by means of targeted mutagenesis, and structural investigation of the central part of SLP65 is needed to identify possible structural transitions.

\subsection{The role of the SLP65 $\mathrm{N}$ terminus in the membrane targeting of SLP65}

The N-terminal region of SLP65 is characterized by a high abundance of positively charged and hydrophobic amino acids. This region is remarkably conserved between species, with more than $90 \%$ sequence identity at the protein level. The profoundly conserved nature of the SLP65 N terminus is indicative of its structural and/or functional importance.

Indeed, as it was first demonstrated by Kohler et al. in 2005 , the $\mathrm{N}$ terminus is essential for the function and membrane targeting of SLP65. The studies by my colleagues and me have confirmed that the $\mathrm{N}$ terminus is essential for the membrane association of SLP65, but our investigations point to a different molecular mechanism than that originally proposed by Koehler et al. A substitution of the residue 125, central to the proposed motif, with other aliphatic amino acids did not interfere with SLP65 function; excluding a functional leucine zipper (see Figure 4.9). Moreover, substitutions of those hydrophobic residues not corresponding to the heptad repeat of the leucine zipper, such as M21, V22, M33 and 136 to $A$ and $G$, resulted in a strongly diminished $\mathrm{Ca}^{2+}$ flux and defective plasma membrane recruitment of SLP65 in DT40 cells (see Figure 4.23). This indicates a general requirement for the hydrophobic environment in the SLP65 N terminus.

My results point to a function of the SLP65 $\mathrm{N}$ terminus in membrane association via direct lipid binding. It was previously found in our group that the N terminus of SLP65 can bind to PIP-spotted membranes (PIP strips). It was hypothesized that this part of SLP65 constitutes a PIP-binding motif, which interacts with negatively charged lipids via electrostatic interactions (Herrmann, 2009). To confirm this finding, I used liposome floatation assays. In this method, PIPs are incorporated into a lipid bilayer, which provides a more "native" binding interface. I found that the SLP65 $\mathrm{N}$ terminus binds neutral liposomes independent of PIPs. Exchange of L18 to $\mathrm{K}$ abolished this interaction (see Figure $4.13 \mathrm{C}$ ), indicating the importance of hydrophobic residues in mediating lipid binding. This has been further confirmed by NMR analysis of the isolated $N$ terminus $\left(\mathrm{SLP}_{5-50}\right)$ in the presence of neutral liposomes. This analysis has identified the residues important for the liposome association - among them L18 (see Figure $4.21 \mathrm{C}$ ). 
Together with my colleagues, Dr. M. Engelke and J. Kühn, we found that the $\mathrm{N}$ terminus confers membrane association to SLP65 already in resting DT40 cells, prior to any BCR stimulation. Constitutive association of the $\mathrm{N}$ terminus with membranes was already reported by Kohler et al.. However, they reported that SLP65 associates with the plasma membrane, and we, in contrast, argue that the vesicular membranes are targeted by SLP65 in resting cells. Detailed microscopic analysis revealed that the association of SLP65 with vesicles is reversible, as evident from the high fluorescence recovery rate upon photobleaching of vesicle-resident Citrine-tagged SLP65 (Figure 4.11). The cytosolic pool of SLP65 is also larger than the vesicular pool, as can be observed from fractionation studies and microscopic analysis. These observations indicate that the vesicle-SLP65 association in resting cells is weak. This is consistent with the results of the liposome floatation experiments, which show that the affinity of the $\mathrm{N}$ terminus for lipids is not very high, at least when neutral lipid species are concerned. The intrinsic disorder and the lack of structural transition in the $\mathrm{N}$ terminus upon lipid binding further support the hypothesis that the SLP65-vesicle association is mediated by weak hydrophobic interactions.

However, even this weak association seems to be critical for signaling, since the inactivation of the $\mathrm{N}$ terminus by mutations that shift the equilibrium towards the cytosolic fraction results in a signaling-incompetent SLP65, which cannot translocate to the plasma membrane and initiate the release of intracellular $\mathrm{Ca}^{2+}$ (see Figure 4.9 and 4.23). The vesicle residence of SLP65 in resting cells therefore appears to help the protein to get to the plasma membrane upon BCR stimulation. The easiest explanation for this observation is the use of vesicular transport by SLP65. Indeed, interference with vesicular trafficking by means of primaquine, a substance that inhibits budding of vesicles from donor membranes (Hiebsch et al., 1991), led to the inhibition of BCR signaling in DT40 cells (Engelke et al., 2014). Our studies therefore point towards a mechanism where the $\mathrm{N}$ terminus mediates association of SLP65 with vesicles in resting cells, which are transported to the plasma membrane and deliver SLP65 to the BCR upon antigen binding (see Figure 5.1). 


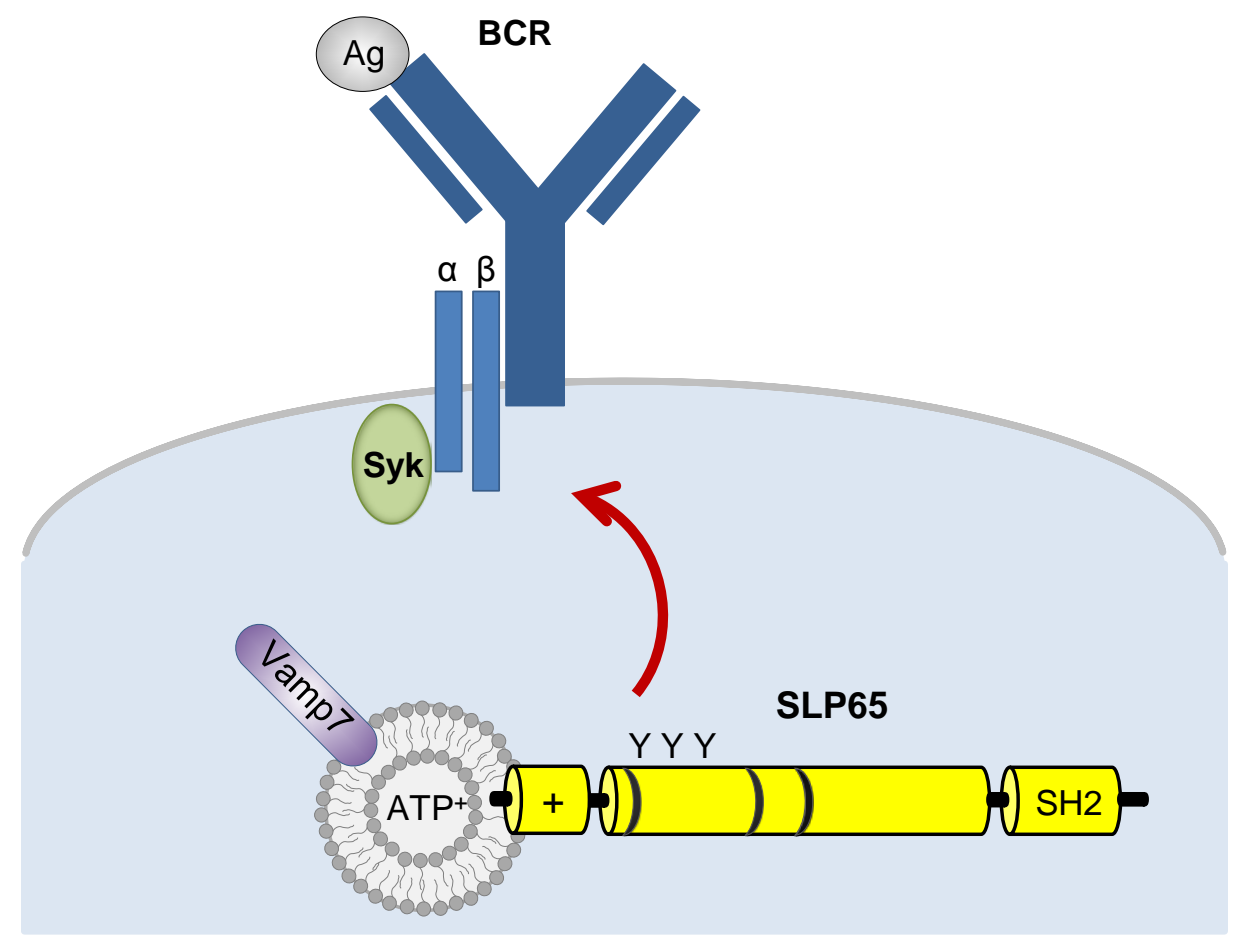

Figure 5.1 The model of SLP65 translocation to the plasma membrane on intracellular vesicles.

The $\mathrm{N}$ terminus mediates the association of SLP65 with exosome-like vesicles in resting B cells. The vesicles carry the SNARE protein Vamp7 and are positive for ATP (detectable by quinacrinestaining). Vesicular transport delivers SLP65 to the BCR upon activation.

The vesicular route might not be the only mechanism for the SLP65 membrane translocation in B cells. Actually, my results indicate that in certain cases SLP65 can be recruited to the plasma membrane in an $\mathrm{N}$ terminus-independent manner. We investigated the vesicular residence of SLP65 in an immature B cell line DT40. Analysis of the subcellular localization of SLP65 in a mature human DG75 B cell line showed that the intracytoplasmic dot-like distribution of SLP65 is discernable, but not as prominent as in DT40 cells. Colocalization experiments with quinacrine have indicated that these intracellular speckles indeed represent the vesicular population of SLP65 (data not shown). However, the phenotype of the $\mathrm{N}$ terminus inactivation via deletion or amino acid exchanges was not as strong in DG75 cells as in DT40 cells. All single point substitution variants of SLP65 mounted an only slightly diminished $\mathrm{Ca}^{2+}$ response upon BCR stimulation even though the plasma membrane recruitment of SLP65 was compromised (see Figure 4.24). Similarly, s/p65\% mouse primary cells reconstituted with the $\Delta N$ and L18K variants of SLP65 mounted almost normal $\mathrm{Ca}^{2+}$ responses (see Figure 4.25). From this data, I conclude that the requirement for the SLP65 N terminus is different at different 
developmental stages and the mechanism of SLP65 translocation via the vesicular route is more important for immature than for mature $B$ cells. This is consistent with the finding by Koehler et al, who showed that the N terminus of SLP65 is essential for the pre-BCR signaling and for the developmental transition from the pre-B cell to the immature $B$ cell stage (Kohler et al., 2005). The minor dependence of mature B cell types on the SLP65 N terminus implies that an alternative pathway regulates the membrane targeting of SLP65. It is possible that a regulator, expressed in a developmental stage-specific manner, inhibits the vesicular pathway or enhances alternative pathways of SLP65 recruitment to the plasma membrane in mature B cells. This regulator could be a direct SLP65 interaction partner, or e.g. an enzyme modifying the lipid composition of the vesicles, making the attachment of SLP65 less/more likely. However, it is also possible that the differences in the requirement for the SLP65 $\mathrm{N}$ terminus are species-specific. In chicken, mouse and human there could be alternative molecules expressed which regulate the SLP65 membrane targeting.

\subsection{The proposed mechanism used by the SLP65 $\mathrm{N}$ terminus for membrane anchoring}

In this thesis I have identified a previously unknown interaction of the SLP65 N terminus with lipids. I showed that SLP65 associates with neutral liposomes independent of lipid composition, but has a higher affinity for negatively charged vesicles. Lipid binding by the $\mathrm{N}$ terminus is further specified by a much higher affinity for small vs. large vesicles, indicating SLP65 can recognize membrane curvature. This was further corroborated in vivo by the functional substitution of the $\mathrm{N}$ terminus with a curvature-sensitive $\mathrm{N}$-BAR domain of Amphyphisin. My data indicates that the $N$ terminus targets SLP65 to small negatively charged vesicles by virtue of reversible association with lipids. The involvement of hydrophobic residues for this interaction, as verified by our NMR analysis, suggests insertion of hydrophobic side chains into the lipid bilayer. However, the contribution of positively charged residues is also likely, since the $\mathrm{N}$ terminus bound stronger to negatively charged liposomes.

Unlike many peripheral membrane proteins, which possess lipid-binding domains able to recognize certain phospholipid species, SLP65 doesn't appear to have such a specific lipid-binding pocket and rather adheres to all negatively charged membranes. This electrostatic interaction with the membranes is therefore not very specific. Curvature recognition might contribute more to specificity, since SLP65 preferentially binds to the 
liposomes with a radius of $20 \mathrm{~nm}$, rather than to the liposomes with a radius of $60 \mathrm{~nm}$ (see Figure 4.15). Two mechanisms of curvature sensing used by other proteins have been described. One is the recognition of the geometric shape of the membrane, as is exemplary of BAR domains, which have a crescent-shaped fold that binds to membranes of certain curvature. The other mechanism is the recognition of lipid-packing defects, as is typical of ALPS motifs, which are intrinsically disordered sequences that form amphipathic a-helices on the membrane and insert the hydrophobic side chains between the loosely spaced lipids (for review, see Antonny, 2011). The N terminus of SLP65 is more likely to use the latter mechanism, given its relatively small size, disordered nature in solution, and the predicted propensity to form an amphipathic a-helix (prediction by AmphipaSeek; Sapay et al., 2006). However, no structural transition in the isolated $\mathrm{N}$ terminus was observed upon binding to SUVs (see Figure 4.21 and 4.22). Nevertheless, even in a disordered conformation curvature recognition could be mediated by the insertion of the hydrophobic side chains into the lipid bilayer. This is easier in a small vesicle, where the lipids are further apart than in a large vesicle with a more flat surface and closely packed lipids (Figure 5.2). The curvature of intracellular membranes is affected by their lipid composition. The presence of lipids with a small head group area, such as PE or DAG, has been shown to induce positive curvature by creating lipid packing defects (Vamparys et al., 2013). SLP65 could therefore have a preference for membranes enriched in such conical phospholipids.

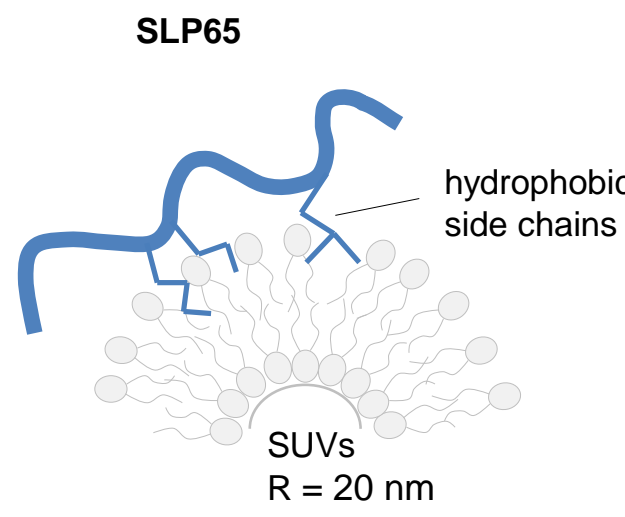

phospholipid
SLP65

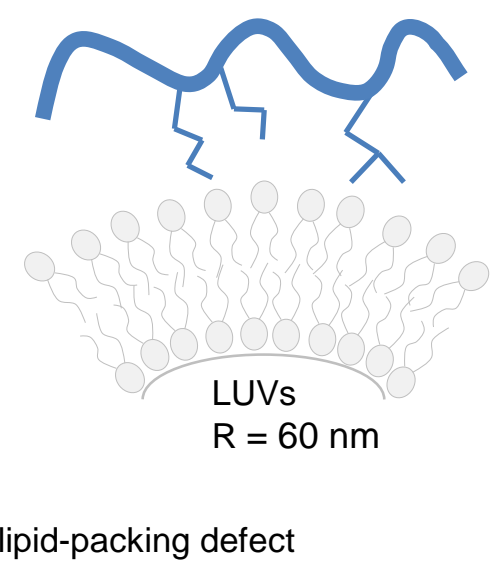

Figure 5.2 The proposed mechanism of curvature recognition by SLP65.

SLP65 senses membrane curvature by detecting lipid-packing defects and inserting hydrophobic side chains between the loosely spaced lipids, thereby penetrating the lipid bilayer. In SUVs lipid heads are spaced further apart, facilitating the binding of the SLP65 N terminus. 
My data indicates that lipid binding might not be the only mechanism used by the SLP65 $\mathrm{N}$ terminus for membrane targeting. Single amino acid substitutions in the $\mathrm{N}$ terminus did not abolish lipid binding, even though they compromised the plasma membrane recruitment of SLP65 (see Figure $4.23 \mathrm{D}$ ). The most plausible explanation is membrane anchoring by an alternative mechanism, such as via binding to a vesicle-resident protein, oligomerization, or lipid modification which promotes insertion of the $\mathrm{N}$ terminus into the membrane. Existence of a protein ligand for the SLP65 $\mathrm{N}$ terminus cannot be excluded, but the interaction partners which were described, such as BNAS2 (Imamura et al., 2004) or CaM (Herrmann, 2009), appear to be irrelevant for the membrane translocation process. Oligomerization of SLP65 via the $\mathrm{N}$ terminus has been proposed, but no experimental evidence for this hypothesis could be obtained (Engelke et al., 2014 and unpublished data from the lab).

It is, however, also possible that the individual amino acid exchanges reduced the lipidbinding affinity of the SLP65 N terminus. This change could not be detected by the "semiquantitative" method of liposome floatation, but was sufficient to compromise membrane targeting in the cell. The investigation of the lipid-binding affinity of SLP65 with quantitative methods, such as ITC (isothermal titration calorimetry), FCS (fluorescence correlation spectroscopy) or NMR, will be useful to confirm the relevance of the SLP65-lipid interaction in living cells and might explain the phenotypes of the single point variants.

There might be yet another explanation as to why some of the single point mutants of SLP65 failed to initiate BCR signaling. One should note that exchanges of some amino acids, especially residues $\mathrm{I} 36$ and $\mathrm{L} 39$ for $\mathrm{A}, \mathrm{G}$ or $\mathrm{K}$, resulted in a strong nuclear localization of SLP65, as visualized by CLSM (see Figure $4.23 \mathrm{~B}$ ). This mis-localization could account for the insufficiency of SLP65 in the cytoplasm and at the vesicles, which I term the "dilution effect". This would explain the inability of these variants to reach the plasma membrane upon BCR ligation. This observation further supports the general notion that the $\mathrm{N}$ terminus regulates subcellular localization of SLP65 in resting cells. But why is SLP65 imported into the nucleus? Nuclear localization signals (NLSs) are short stretches of basic amino acids, which are recognized and bound by importins, proteins mediating the nuclear import (Marfori et al., 2011). The NLS sequences are diverse and not very specific. The subcellular localization of proteins is further specified by the presence (or absence) of nuclear export signals (NES), which are bound by exportins and direct proteins out of the nucleus. Abundance of lysine and arginine residues in SLP65 could lead to the nuclear import "by default", i.e. due to the unspecific binding of the 
positively charged residues by importins. However, since SLP65 is not found in the nucleus under normal conditions, it needs a NES site to be exported out of the nucleus. The classical leucine-rich NES consensus sequence, recognized by the exportin CRM1, is $\varphi-X(2,3)-\varphi-X(2,3)-\varphi-X-\varphi$, where $\varphi$ is $L, V, I, M$ or $F$, and $X(2,3)$ represents any two or three amino acids (Dong et al., 2009). The sequence 32-IMNKIKKLKV-39 in SLP65 fits this pattern and could function as a NES site. Inactivation of the NES motif by substitution of the critical residues 132, I36 or L39 would prevent recognition by CRM1 and nuclear export, which explains the nuclear localization of the respective single point mutants.

I conclude that the $\mathrm{N}$ terminus targets SLP65 to vesicles by virtue of direct lipid binding. However, lipid binding alone might be insufficient for stable vesicle association. It is likely that another mechanism exists, such as dimerization or interaction with a transmembrane protein, which further stabilizes this interaction.

\subsection{The $\mathrm{N}$ terminus cooperates with CIN85 for the efficient membrane targeting of SLP65}

Peripheral membrane proteins, which adhere to membrane surfaces from the cytosol, are surrounded by tens of millimolar of lipids. This high value implies that a high affinity is not needed for membrane association; instead the specificity of binding is important, i.e. the protein must be able to distinguish one membrane compartment from another (Bigay \& Antonny, 2012). In case of SLP65, the specificity is moderate: curved and negatively charged membranes are targeted by the lipid-binding motif in the $\mathrm{N}$ terminus. In that case, how does it tether SLP65 to a particular vesicle species inside the cell?

It is possible that additional specificity is provided by other domains or interaction partners of SLP65. I propose a model, where first, the $\mathrm{N}$ terminus concentrates SLP65 at all negatively charged curved membranes. Then, binding of SLP65 to a vesicle-resident ligand enriches SLP65 at a particular type of vesicle (see Figure 5.3). This ligand could be CIN85 or another binding partner of SLP65. I and my colleagues showed that the CIN85 binding is indeed required for the vesicular localization of SLP65. The CIN85 bindingdeficient variant, SLP65 M23, is distributed in the cytosol of resting DT40 cells similarly to the SLP65 $\Delta N$ (Engelke et al., 2014). This is also valid for the N-BAR-SLP65 chimeric protein with inactivated CIN85-binding sites, which is also not localized at intracellular vesicles and cannot activate $\mathrm{Ca}^{2+}$ mobilization (see Figure 4.18). Hence, targeting SLP65 to small negatively charged vesicles is insufficient for signaling and needs to be supported by CIN85 binding. 
It was shown by J. Kühn that the CC domain of CIN85 can substitute the SLP65 N terminus for membrane targeting. The CC domain may anchor CIN85 to membranes via its interaction with phosphatidic acid. I confirmed this interaction with liposome floatation assays. However, interfering with the PA metabolism by inhibition of the PA-producing enzymes PLD1 and PLD2 did not affect the association of CIN85 with membranes (data not shown). Moreover, the signaling-incompetent mutant variant of the $\mathrm{CC}$ domain could still associate with the PA-containing liposomes, indicating that PA binding is not the essential function of the $\mathrm{CC}$ domain (unpublished data, in collaboration with J. Kühn). The CC domain of CIN85 is also implicated in protein hetero- and homo-oligomerization (Gaidos et al., 2007). This could bring several SLP65 molecules together and amplify BCR signaling. Therefore, CIN85 binding may both navigate and amplify the signaling by SLP65. The exact contribution of CIN85 to SLP65 activation was investigated by J. Kühn in his doctoral work.

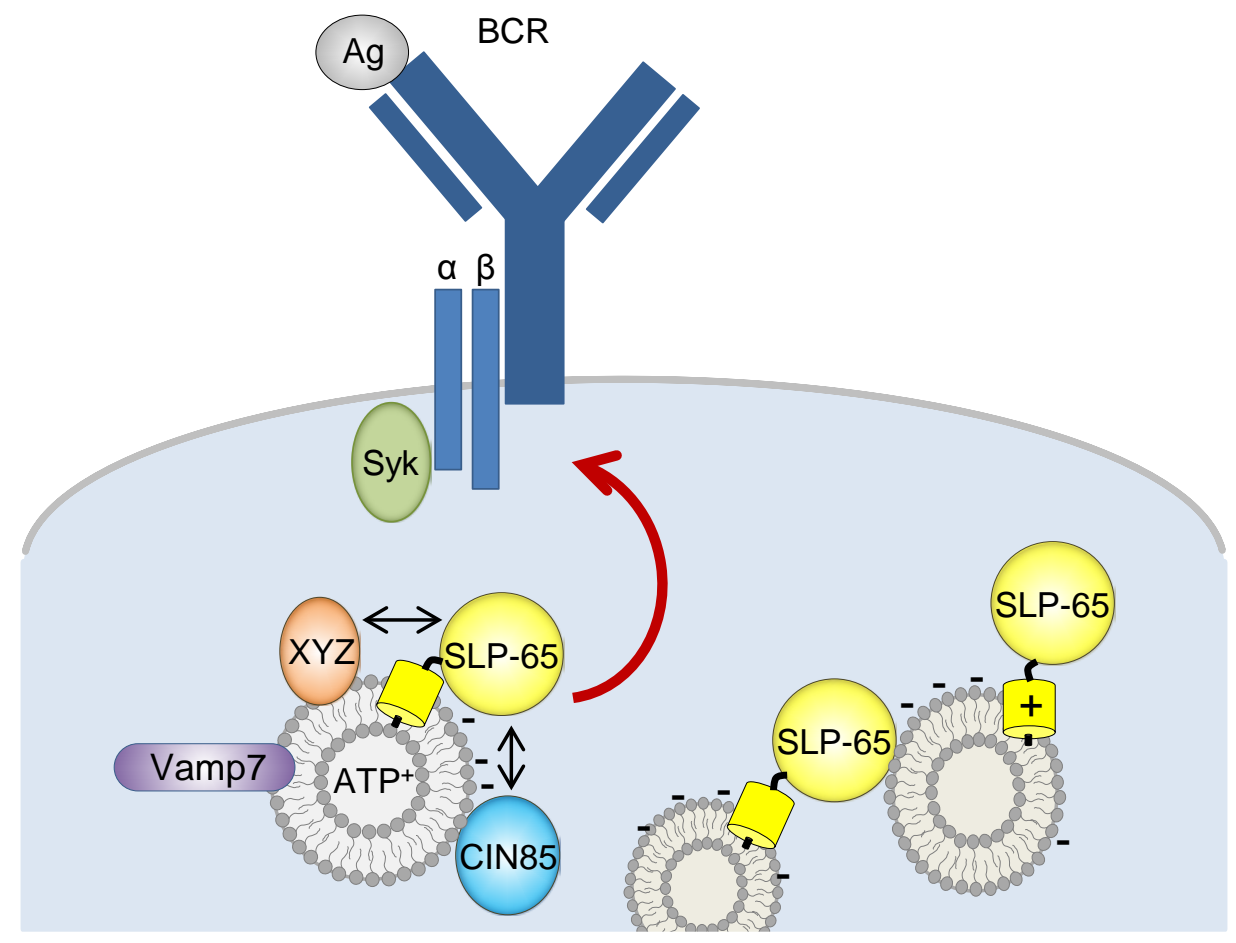

Figure 5.3 The model of vesicle targeting by the SLP65 $\mathrm{N}$ terminus.

The $\mathrm{N}$ terminus adheres to negatively charged curved membranes. The specificity is provided by the binding to CIN85 and/or to a hypothetical vesicle-resident protein XYZ. The vesicles are transported to the plasma membrane upon BCR stimulation and deliver the associated signaling proteins to the activated BCR. 


\subsection{The vesicular route for the plasma membrane targeting of signaling proteins in lymphocytes}

Our results indicated that the association of SLP65 with vesicles is essential for BCR signaling. The advantage of this mechanism could be a more efficient signaling initiation, since instead of being directly recruited from the cytosol to the plasma membrane, signaling proteins can be recruited as part of the pre-assembled signaling complexes. This is especially useful for adaptor proteins, which, unlike enzymes, cannot activate several targets at a time and therefore rely on direct or indirect oligomerization.

The vesicle-mediated recruitment of signaling proteins to the plasma membrane could be a general mechanism not specific for B cells, but also used by other receptor systems. Indeed, there is emerging evidence that similar mechanisms operate in T lymphocytes. Signaling downstream of the TCR depends on the integral plasma membrane protein Lat. Recently this adaptor protein was also detected on intracellular vesicles (Bonello et al., 2004). In a study using immobilized T cell-APC conjugates, Lat-containing vesicles were found to move rapidly between the surface microclusters containing adaptor protein SLP76 at the immunological synapse. The motility of these vesicles decreased and Lat phosphorylation took place in the proximity of the SLP76-containing microclusters (Purbhoo et al., 2010). Other TCR signaling proteins, such as Lck and TCRろ, were also described to reside in the exocytotic vesicular compartments, yet distinct from those containing Lat. The adaptor protein SLP76 was not detected at intracellular vesicles. However, its phosphorylation and distribution at the immunological synapse was affected by the inhibition of vesicular traffic and fusion (Soares et al., 2013). The mechanisms of vesicular trafficking in T lymphocytes are just beginning to be unravelled. Recently, the SNARE protein Vamp7 was discovered to be required for the recruitment of Lat-containing vesicles to the TCR and for the subsequent activation of T cells (Larghi et al., 2013).

We also observed colocalization of SLP65 with Vamp7 in DT40 B cells (Figure 4.10 and Engelke et al., 2014). B and T lymphocytes may therefore use similar mechanisms for the transport of vesicle-associated signalling proteins to the cell surface. In support of this hypothesis, modification of SLP76 by the addition of the SLP65 N terminus and the CIN85-binding sites enabled it to fully reconstitute $\mathrm{Ca}^{2+}$ signaling in s/p65 $5^{--}$DT40 cells (unpublished data from Dr. M. Engelke). It is therefore plausible to assume that SLP65 and SLP76 both can engage in the vesicular route of membrane targeting, although they use different anchors to attach themselves to the vesicles. 
No significant colocalization of SLP65 was observed with any of the Golgi and endosomal markers tested. In fact, SLP65 was found to colocalize with only two vesicular markers Vamp7 and quinacrine (Engelke et al., 2014). Vamp7, also known as TI-VAMP, is a vesicle ( $v$-) SNARE protein, which was linked to both endocytic and exocytic pathways, depending on the cell type (Martinez-Arca et al., 2000; Oishi et al., 2006; Krzewski et al., 2011). We analysed the subcellular distribution of Vamp7 in DT40 cells with cryo-electron microscopy in collaboration with the research group of Prof. H. Stark and found that Vamp7 marks multiple vesicle types of a wide size range in DT40 B cells (data not shown). This observation indicated that Vamp7 is not a very specific marker for the SLP65-containing vesicles and is consistent with an only moderate degree of colocalization between the two proteins (Mander's coefficient $\sim 0.2$, i.e. $20 \%$ colocalization). Another exosomal vesicular marker that characterised SLP65-positive vesicles was the dye quinacrine, which stains intracellular ATP. ATP has been shown to act as a paracrine/autocrine signaling molecule in several cell types (for review, see Corriden \& Insel, 2010). In B lymphocytes, ATP is stored in intracellular secretory granules and released upon ligation of the BCR or the Toll-like receptor (TLR). Extracellular ATP is then hydrolysed by enzymes in the plasma membrane into adenosine, which induces class switch recombination in B cells (Schena et al., 2013). It is unlikely that the plasma membrane targeting of SLP65 is coupled to this process, since we used the immature DT40 B cell line for the colocalization analysis, which does not undergo class-switch recombination. However, it is possible that intracellular ATP, transported to the plasma membrane in SLP65-containing vesicles, likewise plays a signalling role for another B cell response, such as e.g. apoptosis in DT40 cells. Interestingly, the release of Lat- and TCR $\zeta$ - containing vesicles in T lymphocytes, as well as the fusion of the ATP-carrying secretory vesicles in B lymphocytes, was regulated by calcium (Schena et al., 2013; Soares et al., 2013). It is possible that $\mathrm{Ca}^{2+}$, liberated upon $\mathrm{BCR}$ activation, also further stimulates the release of the SLP65-containing vesicles in a positive feedback-regulatory loop.

Even though the origin of SLP65-positive vesicles is still elusive, we have a hint that they originate from the trans-Golgi network (TGN). Fusing the $\mathrm{PH}$ domain of the protein OSBP1, which binds phosphatidylinositol-4-phosphate, a lipid species specifically located on TGN, to $\triangle N$ SLP65 was sufficient to bring it to the plasma membrane and to initiate $\mathrm{Ca}^{2+}$ mobilization upon BCR stimulation (Engelke et al., 2014). This indicates that targeting SLP65 to the Golgi network suffices for signaling. Moreover, this experiment once again shows that the $N$ terminus of SLP65 can be substituted by a lipid-binding 
domain. Interestingly, the loss of CIN85 binding by means of amino acid substitutions in the proline-rich regions of SLP65 resulted in the colocalization of the respective M23 mutant with a TGN marker; indicating that in the absence of CIN85 binding SLP65 is "trapped" in the Golgi network (Engelke et al., 2014). These data further support the notion that CIN85 binding further specifies vesicular targeting of SLP65, which is initiated by the $\mathrm{N}$ terminus. Nevertheless, colocalization analysis with more vesicular markers is needed to characterize and understand the origin of the SLP65-containing vesicles. Moreover, it would be interesting to analyse the subcellular distribution of other BCRsignaling proteins, such as Lyn, Syk, Btk, BCR subunits, etc., to investigate their potential involvement in the vesicular route of plasma membrane targeting.

The mechanisms of vesicular transport in lymphocytes are not clear. It is probable that cytoskeleton reorganization plays a major role for this process. BCR signaling induces cytoskeleton depolymerisation and re-polymerization (Hao \& August, 2005). Moreover, it was shown that alteration of the actin cytoskeleton in $B$ cells by using pharmacological agents that disrupt the actin network, such as Latrunculin $A$ and Cytochalasin $D$, is sufficient to trigger BCR signaling (Treanor et al., 2010). CLSM imaging analysis has also shown that treatment of DT40 cells with Latrunculin B results in a rapid translocation of SLP65 to the plasma membrane (unpublished data from the lab). It is therefore possible that the disruption of the actin cytoskeleton induces the release of intracellular vesicles containing SLP65 and maybe other signaling proteins. In support of this idea, constitutive interaction partners of SLP65, CIN85 and CD2AP, have a link to the actin cytoskeleton. CIN85 and CD2AP interact with CapZ isoforms, proteins involved in capping the barbed end of actin filaments and preventing the actin from depolymerisation (Hutchings et al., 2003). The functional role of these interactions is not clear. CD2AP additionally has several actin-binding sites (Kirsch et al., 1999; Tibaldi \& Reinherz, 2003). Further investigations are therefore needed to understand the contribution of the cytoskeleton reorganization to the vesicular transport and BCR signaling. 


\subsection{Conclusions and perspectives}

Together with my colleagues, I showed that the adaptor protein SLP65, which was previously considered to be a purely cytosolic protein, resides at vesicles in resting $B$ cells and that this vesicle association is important for BCR signalling. I identified the vesicleanchoring device as the N terminus of SLP65, which is a direct lipid-binding module. Our findings point to a mechanism where the adaptors SLP65 and CIN85 are delivered to the plasma membrane via vesicular transport upon BCR ligation. Such a mechanism circumvents recruitment of individual cytosolic proteins from the cytosol and is therefore advantageous for efficient signaling initiation.

The origin and protein composition of the herein identified signaling vesicles remains to be elucidated. Advanced microscopy techniques, such as STED (Stimulated emission depletion) and/or TIRF microscopy, should be applied to visualize the direct movement of the vesicles to the plasma membrane upon BCR stimulation. It would be also interesting to investigate whether the SLP65-positive vesicles fuse with the plasma membrane, as this appears not to be the case for the Vamp7- and Lat-positive vesicles in T lymphocytes (Larghi et al., 2013). In order to characterize the protein composition of the SLP65/CIN85containing vesicles further, they should be separated from other organelles in the cells. This has not been possible so far due to the absence of specific protein markers, which are stably associated with the vesicles. Colocalization analysis with more vesicular markers is therefore necessary to identify further vesicle-resident proteins. Once such markers are identified, biochemical approaches such as protein immunoprecipitation or affinity purification may be used to isolate the specific vesicular species. Alternatively, fluorescence-activated vesicle sorting may be applied for this purpose. Such flow cytometry-based techniques have been used to isolate exocytotic vesicles in other cell types (Cao et al., 2008). The isolated vesicles should then be subjected to mass spectrometry techniques to identify their protein constituents. It would also be interesting to investigate the lipid composition of the SLP65-positive vesicles with TLC (Thin Layer Chromatography) or similar techniques.

The investigation of the SLP65 structure by NMR spectroscopy has shown that SLP65 is intrinsically disordered in the N-terminal and central regions. Structural studies of the fulllength protein, instead of truncated variants, are necessary to verify the present data and to explore potential intramolecular interactions within SLP65. To detect possible structural transitions upon activation, it would be useful to investigate the secondary structure of SLP65 in the presence of its inducible interaction partners. For this purpose, SLP65 
should be phosphorylated in vitro by Syk kinase and the NMR spectra in the presence of Btk and/or PLCy2 SH2 domains should be recorded. Finally, structural investigations of the N-terminal single amino acid substitution variants of SLP65 may be useful to understand their loss-of-function phenotype, despite an unaffected ability to bind lipids. To fully comprehend the plasma membrane recruitment process of SLP65 it is also crucial to study the function of the central proline-rich region of the protein. Further mutational analysis of this part should be performed and respective deletion and single point mutants should be reconstituted into SLP65-deficient cell lines in order to narrow down the critical sequences for signaling. 


\section{Bibliography}

Abudula A, Grabbe A, Brechmann M, Polaschegg C, Herrmann N, Goldbeck I, Dittmann K, Wienands J (2007) SLP-65 signal transduction requires Src homology 2 domainmediated membrane anchoring and a kinase-independent adaptor function of Syk. J Biol Chem 282: 29059-29066

Antonny B (2011) Mechanisms of membrane curvature sensing. Annu Rev Biochem 80: 101-123

Baba TW, Humphries EH (1984) Avian leukosis virus infection: analysis of viremia and DNA integration in susceptible and resistant chicken lines. J Virol 51: 123-130

Baba Y, Hayashi K, Fujii Y, Mizushima A, Watarai H, Wakamori M, Numaga T, Mori Y, lino M, Hikida M, Kurosaki T (2006) Coupling of STIM1 to store-operated Ca2+ entry through its constitutive and inducible movement in the endoplasmic reticulum. Proc Natl Acad Sci U S A 103: 16704-16709

Ben-Bassat H, Goldblum N, Mitrani S, Goldblum T, Yoffey JM, Cohen MM, Bentwich Z, Ramot B, Klein E, Klein G (1977) Establishment in continuous culture of a new type of lymphocyte from a "Burkitt like" malignant lymphoma (line D.G.-75). Int J Cancer 19: 2733

Bergman Y, Cedar H (2004) A stepwise epigenetic process controls immunoglobulin allelic exclusion. Nat Rev Immunol 4: 753-761

Bigay J, Antonny B (2012) Curvature, lipid packing, and electrostatics of membrane organelles: defining cellular territories in determining specificity. Dev Cell 23: 886-895

Bonello G, Blanchard N, Montoya MC, Aguado E, Langlet C, He HT, Nunez-Cruz S, Malissen M, Sanchez-Madrid F, Olive D, Hivroz C, Collette Y (2004) Dynamic recruitment of the adaptor protein LAT: LAT exists in two distinct intracellular pools and controls its own recruitment. J Cell Sci 117: 1009-1016 
Brdicka T, Imrich M, Angelisova P, Brdickova N, Horvath O, Spicka J, Hilgert I, Luskova P, Draber P, Novak P, Engels N, Wienands J, Simeoni L, Osterreicher J, Aguado E, Malissen M, Schraven B, Horejsi V (2002) Non-T cell activation linker (NTAL): a transmembrane adaptor protein involved in immunoreceptor signaling. J Exp Med 196: 1617-1626

Bremes V (2012) CIN85/CD2AP-based protein complexes in B cell antigen receptor signaling. Cellular and Molecular Immunology, Georg-August University of Göttingen, Göttingen

Bubeck Wardenburg J, Fu C, Jackman JK, Flotow H, Wilkinson SE, Williams DH, Johnson R, Kong G, Chan AC, Findell PR (1996) Phosphorylation of SLP-76 by the ZAP-70 protein-tyrosine kinase is required for T-cell receptor function. J Biol Chem 271: 1964119644

Buerstedde JM, Reynaud CA, Humphries EH, Olson W, Ewert DL, Weill JC (1990) Light chain gene conversion continues at high rate in an ALV-induced cell line. Embo J 9: 921927

Burkhardt AL, Brunswick M, Bolen JB, Mond JJ (1991) Anti-immunoglobulin stimulation of B lymphocytes activates src-related protein-tyrosine kinases. Proc Natl Acad Sci U S A 88: $7410-7414$

Busse RA, Scacioc A, Hernandez JM, Krick R, Stephan M, Janshoff A, Thumm M, Kuhnel K (2013) Qualitative and quantitative characterization of protein-phosphoinositide interactions with liposome-based methods. Autophagy 9: 770-777

Campbell MA, Sefton BM (1992) Association between B-lymphocyte membrane immunoglobulin and multiple members of the Src family of protein tyrosine kinases. Mol Cell Biol 12: 2315-2321

Cao Z, Li C, Higginbotham JN, Franklin JL, Tabb DL, Graves-Deal R, Hill S, Cheek K, Jerome WG, Lapierre LA, Goldenring JR, Ham AJ, Coffey RJ (2008) Use of fluorescenceactivated vesicle sorting for isolation of Naked2-associated, basolaterally targeted exocytic vesicles for proteomics analysis. Mol Cell Proteomics 7: 1651-1667 
Carrasco YR, Batista FD (2006) B cell recognition of membrane-bound antigen: an exquisite way of sensing ligands. Curr Opin Immunol 18: 286-291

Cheng PC, Dykstra ML, Mitchell RN, Pierce SK (1999) A role for lipid rafts in B cell antigen receptor signaling and antigen targeting. J Exp Med 190: 1549-1560

Chin D, Means AR (2000) Calmodulin: a prototypical calcium sensor. Trends Cell Biol 10: 322-328

Chiu CW, Dalton M, Ishiai M, Kurosaki T, Chan AC (2002) BLNK: molecular scaffolding through 'cis'-mediated organization of signaling proteins. Embo J 21: 6461-6472

Chung JB, Silverman M, Monroe JG (2003) Transitional B cells: step by step towards immune competence. Trends Immunol 24: 343-349

Corriden R, Insel PA (2010) Basal release of ATP: an autocrine-paracrine mechanism for cell regulation. Sci Signal 3: re1

Crabtree GR, Olson EN (2002) NFAT signaling: Choreographing the social lives of cells. Cell 109: S67-S79

Di Noia JM, Neuberger MS (2007) Molecular mechanisms of antibody somatic hypermutation. Annu Rev Biochem 76: 1-22

Dong X, Biswas A, Suel KE, Jackson LK, Martinez R, Gu H, Chook YM (2009) Structural basis for leucine-rich nuclear export signal recognition by CRM1. Nature 458: 1136-1141

Dyson $\mathrm{HJ}$, Wright PE (2002) Coupling of folding and binding for unstructured proteins. Curr Opin Struct Biol 12: 54-60

Engelke M, Engels N, Dittmann K, Stork B, Wienands J (2007) $\mathrm{Ca}(2+)$ signaling in antigen receptor-activated B lymphocytes. Immunol Rev 218: 235-246 
Engelke $\mathrm{M}$, Oellerich $\mathrm{T}$, Dittmann $\mathrm{K}$, Hsiao $\mathrm{HH}$, Urlaub $\mathrm{H}$, Serve $\mathrm{H}$, Griesinger $\mathrm{C}$, Wienands J (2013) Cutting edge: feed-forward activation of phospholipase Cgamma2 via C2 domain-mediated binding to SLP65. J Immunol 191: 5354-5358

Engelke M, Pirkuliyeva S, Kuhn J, Wong L, Boyken J, Herrmann N, Becker S, Griesinger C, Wienands J (2014) Macromolecular assembly of the adaptor SLP-65 at intracellular vesicles in resting B cells. Sci Signal 7: ra79

Engels N, Wollscheid B, Wienands J (2001) Association of SLP-65/BLNK with the B cell antigen receptor through a non-ITAM tyrosine of Ig-alpha. Eur J Immunol 31: 2126-2134

Fanger MW, Hart DA, Wells JV, Nisonoff A (1970) Requirement for cross-linkage in the stimulation of transformation of rabbit peripheral lymphocytes by antiglobulin reagents. $J$ Immunol 105: 1484-1492

Feldmann M (1972) Induction of immunity and tolerance in vitro by hapten protein conjugates. I. The relationship between the degree of hapten conjugation and the immunogenicity of dinitrophenylated polymerized flagellin. J Exp Med 135: 735-753

Flaswinkel H, Reth M (1994) Dual role of the tyrosine activation motif of the Ig-alpha protein during signal transduction via the B cell antigen receptor. Embo J 13: 83-89

Fu C, Turck CW, Kurosaki T, Chan AC (1998) BLNK: a central linker protein in B cell activation. Immunity 9: 93-103

Futterer K, Wong J, Grucza RA, Chan AC, Waksman G (1998) Structural basis for Syk tyrosine kinase ubiquity in signal transduction pathways revealed by the crystal structure of its regulatory $\mathrm{SH} 2$ domains bound to a dually phosphorylated ITAM peptide. $\mathrm{J} \mathrm{Mol} \mathrm{Biol}$ 281: $523-537$

Gaidos G, Soni S, Oswald DJ, Toselli PA, Kirsch KH (2007) Structure and function analysis of the CMS/CIN85 protein family identifies actin-bundling properties and heterotypic-complex formation. J Cell Sci 120: 2366-2377 
Gerlach J, Ghosh S, Jumaa H, Reth M, Wienands J, Chan AC, Nitschke L (2003) B cell defects in SLP65/BLNK-deficient mice can be partially corrected by the absence of CD22, an inhibitory coreceptor for BCR signaling. Eur J Immunol 33: 3418-3426

Gold MR, Matsuuchi L, Kelly RB, DeFranco AL (1991) Tyrosine phosphorylation of components of the B-cell antigen receptors following receptor crosslinking. Proc Natl Acad Sci U S A 88: 3436-3440

Goldbeck I (2007) Studien zur subzellulären Navigation von Signalmolekülen der Ca2+Antwort in aktivierten B-Lymphocyten. Biochemistry, University of Bielefeld, Bielefeld

Goodnow CC, Vinuesa CG, Randall KL, Mackay F, Brink R (2010) Control systems and decision making for antibody production. Nat Immunol 11: 681-688

Gorska MM, Liang Q, Karim Z, Alam R (2009) Uncoordinated 119 protein controls trafficking of Lck via the Rab11 endosome and is critical for immunological synapse formation. J Immunol 183: 1675-1684

Greenfield NJ (2006) Using circular dichroism spectra to estimate protein secondary structure. Nat Protoc 1: 2876-2890

Grynkiewicz G, Poenie M, Tsien RY (1985) A new generation of Ca2+ indicators with greatly improved fluorescence properties. J Biol Chem 260: 3440-3450

Hao S, August A (2005) Actin depolymerization transduces the strength of B-cell receptor stimulation. Mol Biol Cell 16: 2275-2284

Hashimoto S, Iwamatsu A, Ishiai M, Okawa K, Yamadori T, Matsushita M, Baba Y, Kishimoto T, Kurosaki T, Tsukada S (1999) Identification of the $\mathrm{SH} 2$ domain binding protein of Bruton's tyrosine kinase as BLNK--functional significance of Btk-SH2 domain in B-cell antigen receptor-coupled calcium signaling. Blood 94: 2357-2364

Hempel WM, Schatzman RC, DeFranco AL (1992) Tyrosine phosphorylation of phospholipase C-gamma 2 upon cross-linking of membrane Ig on murine B lymphocytes. J Immunol 148: 3021-3027 
Herrmann N (2009) Kooperation funktioneller Domänen des Adapterproteins SLP-65 für die Ca2+ -Antwort in B-Lymphocyten. Biochemistry, Bielefeld University, Bielefeld

Hiebsch RR, Raub TJ, Wattenberg BW (1991) Primaquine blocks transport by inhibiting the formation of functional transport vesicles. Studies in a cell-free assay of protein transport through the Golgi apparatus. J Biol Chem 266: 20323-20328

Honjo T, Kinoshita K, Muramatsu M (2002) Molecular mechanism of class switch recombination: linkage with somatic hypermutation. Annu Rev Immunol 20: 165-196

Hutchings NJ, Clarkson N, Chalkley R, Barclay AN, Brown MH (2003) Linking the T cell surface protein CD2 to the actin-capping protein CAPZ via CMS and CIN85. J Biol Chem 278: $22396-22403$

Imamura Y, Katahira T, Kitamura D (2004) Identification and characterization of a novel BASH N terminus-associated protein, BNAS2. J Biol Chem 279: 26425-26432

Ishiai M, Kurosaki M, Inabe K, Chan AC, Sugamura K, Kurosaki T (2000) Involvement of LAT, Gads, and Grb2 in compartmentation of SLP-76 to the plasma membrane. $J$ Exp Med 192: 847-856

Ishiai M, Kurosaki M, Pappu R, Okawa K, Ronko I, Fu C, Shibata M, Iwamatsu A, Chan AC, Kurosaki T (1999a) BLNK required for coupling Syk to PLC gamma 2 and Rac1-JNK in B cells. Immunity 10: 117-125

Ishiai M, Sugawara H, Kurosaki M, Kurosaki T (1999b) Cutting edge: association of phospholipase C-gamma 2 Src homology 2 domains with BLNK is critical for B cell antigen receptor signaling. J Immunol 163: 1746-1749

Janssen E, Zhu M, Zhang W, Koonpaew S (2003) LAB: a new membrane-associated adaptor molecule in B cell activation. Nat Immunol 4: 117-123 
Johmura S, Oh-hora M, Inabe K, Nishikawa Y, Hayashi K, Vigorito E, Kitamura D, Turner M, Shingu K, Hikida M, Kurosaki T (2003) Regulation of Vav localization in membrane rafts by adaptor molecules Grb2 and BLNK. Immunity 18: 777-787

Jumaa H, Wollscheid B, Mitterer M, Wienands J, Reth M, Nielsen PJ (1999) Abnormal development and function of $B$ lymphocytes in mice deficient for the signaling adaptor protein SLP-65. Immunity 11: 547-554

Kabak S, Skaggs BJ, Gold MR, Affolter M, West KL, Foster MS, Siemasko K, Chan AC, Aebersold R, Clark MR (2002) The direct recruitment of BLNK to immunoglobulin alpha couples the B-cell antigen receptor to distal signaling pathways. Mol Cell Biol 22: 25242535

Kaisho T, Takeda K, Tsujimura T, Kawai T, Nomura F, Terada N, Akira S (2001) IkappaB kinase alpha is essential for mature B cell development and function. $J$ Exp Med 193: 417426

Kim S, Humphries EH, Tjoelker L, Carlson L, Thompson CB (1990) Ongoing diversification of the rearranged immunoglobulin light-chain gene in a bursal lymphoma cell line. Mol Cell Biol 10: 3224-3231

Kirsch KH, Georgescu MM, Ishimaru S, Hanafusa H (1999) CMS: an adapter molecule involved in cytoskeletal rearrangements. Proc Natl Acad Sci U S A 96: 6211-6216

Kohler F, Storch B, Kulathu Y, Herzog S, Kuppig S, Reth M, Jumaa H (2005) A leucine zipper in the $\mathrm{N}$ terminus confers membrane association to SLP-65. Nat Immunol 6: 204210

Kometani K, Yamada T, Sasaki Y, Yokosuka T, Saito T, Rajewsky K, Ishiai M, Hikida M, Kurosaki T (2011) CIN85 drives B cell responses by linking BCR signals to the canonical NF-kappaB pathway. J Exp Med 208: 1447-1457

König L (2012) B Cell Antigen Receptor-intrinsic Costimulation of IgG and IgE Isotypes. Georg-August University of Goettingen, 
Koretzky GA, Abtahian F, Silverman MA (2006) SLP76 and SLP65: complex regulation of signalling in lymphocytes and beyond. Nat Rev Immunol 6: 67-78

Krzewski K, Gil-Krzewska A, Watts J, Stern JN, Strominger JL (2011) VAMP4- and VAMP7-expressing vesicles are both required for cytotoxic granule exocytosis in NK cells. Eur J Immunol 41: 3323-3329

Kurosaki T, Johnson SA, Pao L, Sada K, Yamamura H, Cambier JC (1995) Role of the Syk autophosphorylation site and $\mathrm{SH} 2$ domains in $\mathrm{B}$ cell antigen receptor signaling. $\mathrm{J}$ Exp Med 182: 1815-1823

Kurosaki T, Maeda A, Ishiai M, Hashimoto A, Inabe K, Takata M (2000) Regulation of the phospholipase C-gamma2 pathway in B cells. Immunol Rev 176: 19-29

Kurosaki T, Shinohara H, Baba Y (2010) B cell signaling and fate decision. Annu Rev Immunol 28: 21-55

Laemmli UK (1970) Cleavage of structural proteins during the assembly of the head of bacteriophage T4. Nature 227: 680-685

Landsteiner K (1924) Experiments on Anaphylaxis to Azoproteins. J Exp Med 39: 631-637

Larghi P, Williamson DJ, Carpier JM, Dogniaux S, Chemin K, Bohineust A, Danglot L, Gaus K, Galli T, Hivroz C (2013) VAMP7 controls T cell activation by regulating the recruitment and phosphorylation of vesicular Lat at TCR-activation sites. Nat Immunol 14: 723-731

Liu SK, Fang N, Koretzky GA, McGlade CJ (1999) The hematopoietic-specific adaptor protein gads functions in T-cell signaling via interactions with the SLP-76 and LAT adaptors. Curr Biol 9: 67-75

Lupas A, Van Dyke M, Stock J (1991) Predicting coiled coils from protein sequences. Science 252: 1162-1164 
Mackay F, Schneider P, Rennert P, Browning J (2003) BAFF AND APRIL: a tutorial on B cell survival. Annu Rev Immunol 21: 231-264

Marfori M, Mynott A, Ellis JJ, Mehdi AM, Saunders NF, Curmi PM, Forwood JK, Boden M, Kobe B (2011) Molecular basis for specificity of nuclear import and prediction of nuclear localization. Biochim Biophys Acta 1813: 1562-1577

Martinez-Arca S, Alberts P, Galli T (2000) Clostridial neurotoxin-insensitive vesicular SNAREs in exocytosis and endocytosis. Biol Cell 92: 449-453

Melchers F, ten Boekel E, Seidl T, Kong XC, Yamagami T, Onishi K, Shimizu T, Rolink AG, Andersson J (2000) Repertoire selection by pre-B-cell receptors and B-cell receptors, and genetic control of B-cell development from immature to mature B cells. Immunol Rev 175: $33-46$

Metzger H (1992) Transmembrane signaling: the joy of aggregation. J Immunol 149: 1477-1487

Minegishi Y, Rohrer J, Coustan-Smith E, Lederman HM, Pappu R, Campana D, Chan AC, Conley ME (1999) An essential role for BLNK in human B cell development. Science 286: 1954-1957

Minguet S, Dopfer EP, Schamel WW (2010) Low-valency, but not monovalent, antigens trigger the B-cell antigen receptor (BCR). Int Immunol 22: 205-212

Motto DG, Ross SE, Wu J, Hendricks-Taylor LR, Koretzky GA (1996) Implication of the GRB2-associated phosphoprotein SLP-76 in T cell receptor-mediated interleukin 2 production. J Exp Med 183: 1937-1943

Murphy K (2011) Janeway's Immunobiology, 8th edition: Garland Science.

Niiro H, Clark EA (2002) Regulation of B-cell fate by antigen-receptor signals. Nat Rev Immunol 2: 945-956 
Oellerich T, Bremes V, Neumann K, Bohnenberger H, Dittmann K, Hsiao HH, Engelke M, Schnyder T, Batista FD, Urlaub H, Wienands J (2011) The B-cell antigen receptor signals through a preformed transducer module of SLP65 and CIN85. Embo J 30: 3620-3634

Oh-hora M, Johmura S, Hashimoto A, Hikida M, Kurosaki T (2003) Requirement for Ras guanine nucleotide releasing protein 3 in coupling phospholipase C-gamma2 to Ras in $\mathrm{B}$ cell receptor signaling. J Exp Med 198: 1841-1851

Oishi Y, Arakawa T, Tanimura A, Itakura M, Takahashi M, Tajima Y, Mizoguchi I, Takuma T (2006) Role of VAMP-2, VAMP-7, and VAMP-8 in constitutive exocytosis from HSY cells. Histochem Cell Biol 125: 273-281

Ottinger EA, Botfield MC, Shoelson SE (1998) Tandem SH2 domains confer high specificity in tyrosine kinase signaling. J Biol Chem 273: 729-735

Pahl HL (1999) Activators and target genes of Rel/NF-kappaB transcription factors. Oncogene 18: 6853-6866

Pappu R, Cheng AM, Li B, Gong Q, Chiu C, Griffin N, White M, Sleckman BP, Chan AC (1999) Requirement for B cell linker protein (BLNK) in B cell development. Science 286: 1949-1954

Parekh AB, Penner R (1997) Store depletion and calcium influx. Physiol Rev 77: 901-930

Patterson HC, Kraus M, Kim YM, Ploegh H, Rajewsky K (2006) The B cell receptor promotes $B$ cell activation and proliferation through a non-ITAM tyrosine in the Igalpha cytoplasmic domain. Immunity 25: 55-65

Patterson RL, Boehning D, Snyder SH (2004) Inositol 1,4,5-trisphosphate receptors as signal integrators. Annu Rev Biochem 73: 437-465

Peng SL, Gerth AJ, Ranger AM, Glimcher LH (2001) NFATc1 and NFATc2 together control both T and B cell activation and differentiation. Immunity 14: 13-20 
Pike KA, Ratcliffe MJ (2005) Dual requirement for the Ig alpha immunoreceptor tyrosinebased activation motif (ITAM) and a conserved non-Ig alpha ITAM tyrosine in supporting Ig alpha beta-mediated B cell development. J Immunol 174: 2012-2020

Pirkuliyeva S (2012) The role of C type lectin 17A in B cell antigen receptor signaling. Cellular and Molecular Immunology, Georg-August University Göttingen, Göttingen

Pleiman CM, D'Ambrosio D, Cambier JC (1994) The B-cell antigen receptor complex: structure and signal transduction. Immunol Today 15: 393-399

Purbhoo MA, Liu H, Oddos S, Owen DM, Neil MA, Pageon SV, French PM, Rudd CE, Davis DM (2010) Dynamics of subsynaptic vesicles and surface microclusters at the immunological synapse. Sci Signal 3: ra36

Putney JW, Jr., Ribeiro CM (2000) Signaling pathways between the plasma membrane and endoplasmic reticulum calcium stores. Cell Mol Life Sci 57: 1272-1286

Reth M (1989) Antigen receptor tail clue. Nature 338: 383-384

Reth M (1992) Antigen receptors on B lymphocytes. Annu Rev Immunol 10: 97-121

Reth M (2001) Oligomeric antigen receptors: a new view on signaling for the selection of lymphocytes. Trends Immunol 22: 356-360

Reth M, Petrac E, Wiese P, Lobel L, Alt FW (1987) Activation of V kappa gene rearrangement in pre-B cells follows the expression of membrane-bound immunoglobulin heavy chains. Embo J 6: 3299-3305

Richards JD, Dave SH, Chou CH, Mamchak AA, DeFranco AL (2001) Inhibition of the MEK/ERK signaling pathway blocks a subset of $B$ cell responses to antigen. $J$ Immunol 166: $3855-3864$

Rowley RB, Burkhardt AL, Chao HG, Matsueda GR, Bolen JB (1995) Syk protein-tyrosine kinase is regulated by tyrosine-phosphorylated Ig alpha/lg beta immunoreceptor tyrosine activation motif binding and autophosphorylation. J Biol Chem 270: 11590-11594 
Saijo K, I M, Santana A, Leitger M, Schmedt C, Tarakhovsky A (2002) Protein kinase C beta controls nuclear factor kappa $B$ activation in $B$ cells through selective regulation of the I kappa B kinase alpha. Journal of Experimental Medicine 195: 1647-1652

Saiki RK, Gelfand DH, Stoffel S, Scharf SJ, Higuchi R, Horn GT, Mullis KB, Erlich HA (1988) Primer-directed enzymatic amplification of DNA with a thermostable DNA polymerase. Science 239: 487-491

Sanchez M, Misulovin Z, Burkhardt AL, Mahajan S, Costa T, Franke R, Bolen JB, Nussenzweig M (1993) Signal transduction by immunoglobulin is mediated through Ig alpha and Ig beta. J Exp Med 178: 1049-1055

Sano R, Hayashi F, Kurosaki C, Yoshida M, Yokoyama S. (2007) Solution structure of the $\mathrm{SH} 2$ domain from mouse b-cell linker protein blnk.

Sapay N, Guermeur Y, Deleage G (2006) Prediction of amphipathic in-plane membrane anchors in monotopic proteins using a SVM classifier. BMC Bioinformatics 7: 255

Saxton TM, van Oostveen I, Bowtell D, Aebersold R, Gold MR (1994) B cell antigen receptor cross-linking induces phosphorylation of the p21ras oncoprotein activators SHC and mSOS1 as well as assembly of complexes containing SHC, GRB-2, mSOS1, and a 145-kDa tyrosine-phosphorylated protein. J Immunol 153: 623-636

Schamel WW, Reth M (2000) Monomeric and oligomeric complexes of the B cell antigen receptor. Immunity 13: 5-14

Scharenberg AM, El-Hillal O, Fruman DA, Beitz LO, Li Z, Lin S, Gout I, Cantley LC, Rawlings DJ, Kinet JP (1998) Phosphatidylinositol-3,4,5-trisphosphate (Ptdlns-3,4,5P3)/Tec kinase-dependent calcium signaling pathway: a target for SHIP-mediated inhibitory signals. Embo J 17: 1961-1972

Schena F, Volpi S, Faliti CE, Penco F, Santi S, Proietti M, Schenk U, Damonte G, Salis A, Bellotti M, Fais F, Tenca C, Gattorno M, Eibel H, Rizzi M, Warnatz K, Idzko M, Ayata CK, Rakhmanov M, Galli T, Martini A, Canossa M, Grassi F, Traggiai E (2013) Dependence of 
immunoglobulin class switch recombination in B cells on vesicular release of ATP and CD73 ectonucleotidase activity. Cell Rep 3: 1824-1831

Schroeder HW, Jr., Cavacini L (2010) Structure and function of immunoglobulins. J Allergy Clin Immunol 125: S41-52

Shinohara H, Yasuda T, Aiba Y, Sanjo H, Hamadate M, Watarai H, Sakurai H, Kurosaki T (2005) PKC beta regulates BCR-mediated IKK activation by facilitating the interaction between TAK1 and CARMA1. Journal of Experimental Medicine 202: 1423-1431

Soares H, Henriques R, Sachse M, Ventimiglia L, Alonso MA, Zimmer C, Thoulouze MI, Alcover A (2013) Regulated vesicle fusion generates signaling nanoterritories that control T cell activation at the immunological synapse. J Exp Med 210: 2415-2433

Sommer K, Guo BC, Pomerantz JL, Bandaranayake AD, Moreno-Garcia ME, Ovechkina YL, Rawlings DJ (2005) Phosphorylation of the CARMA1 linker controls NF-kappa B activation. Immunity 23: 561-574

Stork B, Engelke M, Frey J, Horejsi V, Hamm-Baarke A, Schraven B, Kurosaki T, Wienands J (2004) Grb2 and the non-T cell activation linker NTAL constitute a Ca(2+)regulating signal circuit in B lymphocytes. Immunity 21: 681-691

Su TT, Guo BC, Kawakami Y, Sommer K, Chae K, Humphries LA, Kato RM, Kang S, Patrone L, Wall R, Teitell M, Leitges M, Kawakami T, Rawlings DJ (2002) PKC-beta controls I kappa B kinase lipid raft recruitment and activation in response to BCR signaling. Nat Immunol 3: 780-786

Su YW, Zhang Y, Schweikert J, Koretzky GA, Reth M, Wienands J (1999) Interaction of SLP adaptors with the SH2 domain of Tec family kinases. Eur J Immunol 29: 3702-3711

Takata M, Kurosaki T (1996) A role for Bruton's tyrosine kinase in B cell antigen receptormediated activation of phospholipase C-gamma 2. J Exp Med 184: 31-40 
Teh YM, Neuberger MS (1997) The immunoglobulin (Ig)alpha and Igbeta cytoplasmic domains are independently sufficient to signal B cell maturation and activation in transgenic mice. J Exp Med 185: 1753-1758

Tibaldi EV, Reinherz EL (2003) CD2BP3, CIN85 and the structurally related adaptor protein CMS bind to the same CD2 cytoplasmic segment, but elicit divergent functional activities. Int Immunol 15: 313-329

Timmerman LA, Healy JI, Ho SN, Chen L, Goodnow CC, Crabtree GR (1997) Redundant expression but selective utilization of nuclear factor of activated T cells family members. $J$ Immunol 159: 2735-2740

Tonegawa S (1983) Somatic generation of antibody diversity. Nature 302: 575-581

Treanor B, Depoil D, Gonzalez-Granja A, Barral P, Weber M, Dushek O, Bruckbauer A, Batista FD (2010) The membrane skeleton controls diffusion dynamics and signaling through the B cell receptor. Immunity 32: 187-199

Treanor B, Harwood NE, Batista FD (2009) Microsignalosomes: spatially resolved receptor signalling. Biochem Soc Trans 37: 1014-1018

Trivedi V, Chaudhary N. NPTEL Course "Bioanalytical Techniques and Bioinformatics", Spectroscopic Techniques. Vol. 2014.

Tsang E, Giannetti AM, Shaw D, Dinh M, Tse JK, Gandhi S, Ho H, Wang S, Papp E, Bradshaw JM (2008) Molecular mechanism of the Syk activation switch. J Biol Chem 283: 32650-32659

Tsubata T, Reth M (1990) The products of pre-B cell-specific genes (lambda 5 and VpreB) and the immunoglobulin mu chain form a complex that is transported onto the cell surface. J Exp Med 172: 973-976

Vamparys L, Gautier R, Vanni S, Bennett WF, Tieleman DP, Antonny B, Etchebest C, Fuchs PF (2013) Conical lipids in flat bilayers induce packing defects similar to that induced by positive curvature. Biophys J 104: 585-593 
Von Spee C (2010) Subcellular targeting of adaptor molecules involved in BCR signaling. Cellular and Molecular Immunology, Georg-August University Göttingen, Göttingen

Whitmore L, Wallace BA (2004) DICHROWEB, an online server for protein secondary structure analyses from circular dichroism spectroscopic data. Nucleic Acids Res 32: W668-673

Whitmore L, Wallace BA (2008) Protein secondary structure analyses from circular dichroism spectroscopy: methods and reference databases. Biopolymers 89: 392-400

Wienands J, Freuler F, Baumann G (1995) Tyrosine-phosphorylated forms of Ig beta, CD22, TCR zeta and HOSS are major ligands for tandem SH2 domains of Syk. Int Immunol 7: 1701-1708

Wienands J, Schweikert J, Wollscheid B, Jumaa H, Nielsen PJ, Reth M (1998) SLP-65: a new signaling component in $B$ lymphocytes which requires expression of the antigen receptor for phosphorylation. J Exp Med 188: 791-795

Williams AF, Barclay AN (1988) The immunoglobulin superfamily--domains for cell surface recognition. Annu Rev Immunol 6: 381-405

Winding P, Berchtold MW (2001) The chicken B cell line DT40: a novel tool for gene disruption experiments. J Immunol Methods 249: 1-16

Winslow MM, Gallo EM, Neilson JR, Crabtree GR (2006) The calcineurin phosphatase complex modulates immunogenic B cell responses. Immunity 24: 141-152

Wong J, Ishiai M, Kurosaki T, Chan AC (2000) Functional complementation of BLNK by SLP-76 and LAT linker proteins. J Biol Chem 275: 33116-33122

Xu S, Tan JE, Wong EP, Manickam A, Ponniah S, Lam KP (2000) B cell development and activation defects resulting in xid-like immunodeficiency in BLNK/SLP-65-deficient mice. Int Immunol 12: 397-404 
Yablonski D, Kuhne MR, Kadlecek T, Weiss A (1998) Uncoupling of nonreceptor tyrosine kinases from PLC-gamma1 in an SLP-76-deficient T cell. Science 281: 413-416

Yamamoto T, Yamanashi Y, Toyoshima K (1993) Association of Src-family kinase Lyn with B-cell antigen receptor. Immunol Rev 132: 187-206

Yamanashi Y, Fukui Y, Wongsasant B, Kinoshita Y, Ichimori Y, Toyoshima K, Yamamoto T (1992) Activation of Src-like protein-tyrosine kinase Lyn and its association with phosphatidylinositol 3-kinase upon B-cell antigen receptor-mediated signaling. Proc Natl Acad Sci U S A 89: 1118-1122

Yamanashi Y, Kakiuchi T, Mizuguchi J, Yamamoto T, Toyoshima K (1991) Association of B cell antigen receptor with protein tyrosine kinase Lyn. Science 251: 192-194

Yasuda T, Sanjo H, Pages G, Kawano Y, Karasuyama H, Pouyssegur J, Ogata M, Kurosaki T (2008) Erk kinases link pre-B cell receptor signaling to transcriptional events required for early B cell expansion. Immunity 28: 499-508

Zhang $\mathrm{H}$, Constantine $\mathrm{R}$, Vorobiev $\mathrm{S}$, Chen $\mathrm{Y}$, Seetharaman $\mathrm{J}$, Huang $\mathrm{YJ}$, Xiao R, Montelione GT, Gerstner CD, Davis MW, Inana G, Whitby FG, Jorgensen EM, Hill CP, Tong L, Baehr W (2011) UNC119 is required for G protein trafficking in sensory neurons. Nat Neurosci 14: 874-880

Zhang J, Zheng X, Yang X, Liao K (2009) CIN85 associates with endosomal membrane and binds phosphatidic acid. Cell Res 19: 733-746

Zhang W, Irvin BJ, Trible RP, Abraham RT, Samelson LE (1999) Functional analysis of LAT in TCR-mediated signaling pathways using a LAT-deficient Jurkat cell line. Int Immunol 11: 943-950

Zhang W, Sloan-Lancaster J, Kitchen J, Trible RP, Samelson LE (1998a) LAT: the ZAP70 tyrosine kinase substrate that links T cell receptor to cellular activation. Cell 92: 83-92 
Zhang W, Trible RP, Samelson LE (1998b) LAT palmitoylation: its essential role in membrane microdomain targeting and tyrosine phosphorylation during $\mathrm{T}$ cell activation. Immunity 9: 239-246

Zhang Y (2008) I-TASSER server for protein 3D structure prediction. BMC Bioinformatics 9: 40

Zhou HX (2012) Intrinsic disorder: signaling via highly specific but short-lived association. Trends Biochem Sci 37: 43-48 


\section{Appendix}

\subsection{List of Figures}

Figure 2.1 A simplified scheme of the key signaling events in the BCR signal transduction pathway.

Figure 2.2 Domains and interaction partners of SLP65 involved in membrane targeting. .18

Figure 4.1 SLP65 is an IDP except for the SH2 domain.

Figure 4.2 Amino acid sequence of SLP65 GS.

Figure 4.3 The central part of SLP65 is indispensable for membrane targeting

Figure 4.4 The GS linker in the central part of SLP65 is sufficient for $\mathrm{Ca}^{2+}$ signaling once SLP65 is brought to the BCR.

Figure 4.5 Schematic representations of the Btk- and PLCY2-binding sites on SLP65 and the synthetic construct SLP65 Y96FnewBtk. .59

Figure 4.6 Swapping Btk and PLCy2 binding sites in SLP65 renders it unfunctional. 60

Figure 4.7 The N-terminal region of SLP65 is critical for its function.

Figure 4.8 The putative SLP65-Calmodulin interaction cannot be observed in vivo and the overexpression of CaM does not affect BCR-induced $\mathrm{Ca}^{2+}$ mobilization.

Figure 4.9 Single amino acid exchanges in the putative leucine zipper motif of SLP65 differentially affect $\mathrm{BCR}$-induced $\mathrm{Ca}^{2+}$ mobilization.

Figure 4.10 SLP65 colocalizes with vesicular markers quinacrine and Vamp7 dependent on its $\mathrm{N}$ terminus.

Figure 4.11 Cytosolic and membrane fractions of SLP65 are in constant exchange. .69

Figure 4.12 The purification of His-tagged SLP65 and an overview of the liposome floatation method.

Figure 4.13 SLP65 binds to neutral liposomes dependent on its $\mathrm{N}$ terminus.

Figure 4.14 SLP65 preferentially binds to negatively charged liposomes

Figure 4.15 SLP65 binds preferentially to small liposomes. .75

Figure 4.16 The $\mathrm{N}$ terminus of SLP65 can be substituted by the N-BAR domain of Amphiphysin. .77 
Figure 4.17 N-BAR- $\Delta$ N SLP65 colocalizes with quinacrine-positive vesicles.

Figure 4.18 N-BAR- $\Delta N$ chimera is dependent on CIN85 binding. .80

Figure 4.19 CIN85 CC domain binds to PA-containing liposomes. .81

Figure 4.20 The $\mathrm{N}$ terminus of SLP65 is intrinsically disordered in solution. 83

Figure 4.21 SLP65 $5-50$ binds to SUVs in a defined manner. 85

Figure 4.22 Lipid binding does not induce a structural change in the SLP65 N terminus..86 Figure 4.23 Individual hydrophobic residues in the SLP65 $\mathrm{N}$ terminus are important for correct subcellular localization and signaling, but are dispensable for lipid binding. .88

Figure 4.24 The N terminus of SLP65 is required, but individual hydrophobic residues are dispensable for SLP65 function in DG75 cells. 91

Figure 4.25 The $\mathrm{N}$ terminus of SLP65 is dispensable for $\mathrm{Ca}^{2+}$ signaling in mouse primary B cells. .92

Figure 4.26 The $\mathrm{N}$ terminus cannot function independently of SLP65. 95

Figure 5.1 The model of SLP65 translocation to the plasma membrane on intracellular vesicles. 100

Figure 5.2 The proposed mechanism of curvature recognition by SLP65. 102

Figure 5.3 The model of vesicle targeting by the SLP65 $\mathrm{N}$ terminus. 105

\subsection{List of Tables}

Table 3.1 Cycler parameters for a standard PCR program...... 39

Table 3.2 Cycler parameters for the overlap extension PCR, part 1. 39

Table 3.3 Cycler parameters for the overlap extension PCR, part 2. 40

\subsection{Abbreviations}

$\begin{array}{ll}\alpha & \text { alpha, anti } \\ \text { aa } & \text { Amino acid } \\ \text { Akt1 } & \text { Rac-alpha serine/threonine protein kinase } \\ \text { Ag } & \text { Antigen } \\ \text { ALPS } & \text { Amphipathic lipid packing sensor motif }\end{array}$


AP

APC

ATP

APS

BAR

BASH

Bcl10

BCR

BLNK

bp

BSA

Btk

C2

$\mathrm{CH}$

$\mathrm{CL}$

CaM

CapZ

CARMA1

CC

CCL

CD

cDNA

CD2AP

CD72

CIN85

CIP

Cit

CLEC17A

CLSM
Affinity purification

Antigen presenting cell

Adenine nucleotide phosphate

Ammonium persulphate

Bin-Amphiphysin-Rvs

B cell adaptor protein containing $\mathrm{SH} 2$ domain

B-cell lymphoma/leukemia 10

B cell antigen receptor

B cell linker protein

Base pair

Bovine serum albumin

Bruton's tyrosine kinase

Protein kinase $\mathrm{C}$ conserved region 2

Heavy chain constant domain

Light chain constant domain

Calmodulin

Capping protein (actin filament) muscle Z-line

CARD-containing MAGUK protein 1

Coiled-coil

Cleared cellular lysate

Cluster of differentiation, Circular dichroism

Complementary DNA

CD2-associated protein

Cluster of differentiation 72

Cbl-intereacting protein of $85 \mathrm{kDa}$

Calf intestinal phosphatase

Citrine

C type lectin $17 \mathrm{~A}$

Confocal laser scanning microscopy 
CRD Carbohydrate recognition domain

CRM1 Chromosomal maintenance 1

C-terminal Carboxy-terminal

DAG Diacylglycerol

DLS Dynamic light scattering

DMEM Dulbecco's modified Eagle's medium

DMSO Dimethylsulfoxide

DNA Deoxyribonucleic acid

Dok3 Docking protein 3

DOPC Di-oleyl-phosphatidylcholine

DOPE Di-oleyl-phosphatidylethanolamine

dNTP 2'-deoxynucleoside-5'-triphosphate

DTT 1,4-dithiothretiol

E. coli Escherichia coli

ECL Enhanced chemical luminescence

EGFP Enhanced green fluorescent protein

ER Endoplasmic reticulum

ERK Extracellular signal-regulated kinase

Fab Fragment antigen-binding

FACS Fluorescence activated cell sorter

Fc $\quad$ Fragment crystalisable

FCS Fetal calf serum, fluorescence correlation spectroscopy

FITC Fluorescein isothiocyanate

FRAP Fluorescence recovery after photobleaching

Gads Grb2-related adaptor protein

GEF Guanine nucleotide exchange factor

GFP Green fluorescent protein

Grb2 Growth factor receptor-bound protein 2

GTP Guanine nucleotide phosphate 
GST Glutathione S-transferase

HEPES 2-[4-(2-Hydroxyethyl)-1-piperazinyl]-ethanesulfonic acid

HRPO Horseradish peroxidase

HSQC Heteronuclear single quantum coherence spectrum

Hsv2 Homologous with swollen vacuole phenotype 2

IDP Intrinsically disordered protein

Ig Immunoglobulin

IKB Inhibitor of NF-KB

IKK IKB kinase complex

Indo-1 1H-indole-6-carboxylic acid, 2-[4-[bis[2[(acetyloxy)methoxy]-

IP Immunoprecipitation

IP3 Inositol-1,4,5,-triphosphate

IP3R IP3 receptor

IPTG Isopropyl $\beta$-D-1-thiogalactopyranoside

ITAM Immunoreceptor tyrosine-based activation motif

ITC Isothermal titration calorimetry

Itk IL2-inducible T cell kinase

JNK c-JUN NH2-terminal kinase

LAT Linker for activation of T cells

LB Lysogeny broth

Lck Lymphocyte-specific protein tyrosine kinase

LPS Lipopolysaccharide

LUV Large unilamellar vesicle

Lyn Lck/Yes-related tyrosine kinase

MACS Magnetic-activated cell sorting

MALT1 Mucosa-associated lymphoid tissue lymphoma translocation protein 1

MAPK $\quad$ Mitogen activated protein kinase

MEK1/2 Meiosis-specific serine/threonine kinase 1/2

MHC Major histocompatibility complex 
mlg Membrane-bound immunoglobulin

$\min \quad$ Minute(s)

MLV Multilamellar vesicle

Nck Non-catalytic region of tyrosine kinase

Neo Neomycine

NES Nuclear export signal

NFAT Nuclear factor of activated T cells

NF-kB Nuclear factor for kappa gene in B lymphocytes

NLS Nuclear localization signal

NMR Nuclear Magnetic Resonance

N-terminal Amino-terminal

NTAL Non-T cell activation linker

OD Optical density

OG Oregon Green

OSBP1 Oxysterol-binding protein 1

PA Phosphatidic acid

PAGE Polyacrylamide gel electrophoresis

PBS Phosphate buffered saline

PCR Polymerase chain reaction

PC Phosphatidylcholine

PE Phosphatidylethanolamine

PIK Phosphatidyl inositol kinase

PIP Phosphatidyl inositol phosphate

PIP2 Phosphatidyl-inositol-4,5-bisphosphate

PIP3 Phosphatidyl-inositol-3,4,5-triphosphate

$\mathrm{PH} \quad$ Pleckstrin-homology

PKC $\beta \quad$ Protein kinase C

PLC-y2 Phospholipase C-y2

PLD Phospholipase D 
PRR Proline-rich region

Pre- $\quad$ Precursor

PS Phosphatidylserine

PTK Protein tyrosine kinase

pY Phosphotyrosine

Raf Rat fibrosarcoma

Ras Rat sarcoma

RasGRP3 Ras guanyl-releasing protein 3

RNA Ribonucleic acid

ROI Region of interest

rpm rotations per minute

RPMI Roswell Park Memorial Institute medium

RT Room temperature

SD Standard deviation

SDS Sodium dodecyl sulfate

sec Second

SH2 Src-homology 2

SH3 Src-homology 3

SHP-1 SH2 domain-containing protein tyrosine phosphatase 1

SLP-65 SH2 domain containing leukocyte adaptor protein of $65 \mathrm{kDa}$

SLP-76 SH2 domain containing leukocyte adaptor protein of $76 \mathrm{kDa}$

SNARE Soluble N-ethylmaleimide-sensitive factor

SOC Store-operated $\mathrm{Ca}^{2+}$ channel

Sos $\quad$ Son of sevenless

STED Stimulated emission depletion microscopy

STIM1 Stromal interaction molecule 1

SUV Small unilamellar vesicle

Syk Spleen tyrosine kinase

TAE Tris acetate EDTA buffer 
TAK1 Transforming growth factor beta-activated kinase 1

TALEN Transcription activator-like effector nuclease

TBS Tris buffered saline

TCR T cell antigen receptor

TEMED N,N,N',N'-tetramethylethylene-diamine

TGN Trans-Golgi network

TIRF Total internal reflection microscopy

TLC Thin layer chromatography

TR Texas Red

Tris Tris-(hydroxymethyl)-aminomethane

Triton X- 4-(2',2',4',4'tetramethylbutyl) phenyldecaethyleneglycolether

UV Ultraviolet

VL Light chain variable domain

Vav GDP/GTP exchange factor for Rho family GTPases

VH Heavy chain variable domain

VSV-G Vesicular stomatitis virus glycoprotein

v/v Volume/volume

WT Wild type

w/v Weight/volume

X-gal 5-bromo-4-chloro-3-indolyl- $\beta$-D-galactopyranoside

ZAP-70 $\quad \zeta$-associated protein of $70 \mathrm{kDa}$

\subsection{Amino acids}

$\underline{\text { Amino acid }}$

Alanine

Aspargine

Aspartic acid

Arginine

\section{3 letter code}

Ala

Asn

Asp

Arg

\section{Single letter code}

A

N

D

$\mathrm{R}$ 
Cysteine

Glutamic acid

Glutamine

Glycine

Histidine

Isoleucine

Leucine

Lysine

Methionine

Phenylalanine

Proline

Serine

Threonine

Tryptophan

Tyrosine

Valine
Cys

Glu

GIn

Gly

His

Ile

Leu

Lys

Met

Phe

Pro

Ser

Thr

Trp

Tyr

Val
C

E

Q

G

$\mathrm{H}$

I

L

K

M

F

P

S

T

W

Y

V

\subsection{Deoxyribonucleotides}

Deoxyribonucleotide

Deoxyadenosine monophosphate

Deoxycytidine monophosphate

Deoxyguanine monophosphate

Deoxythymidine monophoshate
Symbol
A
C
G
$\mathrm{T}$ 


\section{Acknowledgements}

First of all, I would like to express my gratitude to my supervisor, Prof. Dr. Jürgen Wienands for giving me the opportunity to carry out my PhD thesis project in his group, for introducing me to the exciting field of molecular immunology, for his guidance, support, constructive criticism and never-exhausting enthusiasm.

I would also like to thank Prof. Dr. Lutz Walter and Professor Dr. Christian Griesinger, as members of my PhD thesis committee, for fruitful discussions and helpful suggestions.

I am very grateful to Dr. Steffen Burkhardt and Kerstin Grüninger from the Molecular Biology program coordination office, for their great support during my $\mathrm{PhD}$ years.

I would like to thank the members of our SFB collaboration from the group of Prof. Griesinger, Dr. Stefan Becker, Claudia Schwiegk, Leo Wong and Dr. Luigi Russo, for stimulating discussions, sharing ideas, performing the NMR experiments and contributing structural data for this project. I'm also grateful for the financial support our project got from DFG via SFB860, and for all the SFB retreats, where I could meet many great scientists and further develop my knowledge.

I would like to thank Dr. Karin Kühnel for introducing me to the liposome-based methods and Prof. Dr. Reinhard Jahn for allowing me to use the resources at the department of Neurobiology to perform my experiments. I'm very grateful to Andreea Scacioc, who taught me the liposome floatation assays, shared her bench with me and was always there to answer my questions and help me with suggestions, thereby greatly contributing to my project. I'm also thankful to Angel Perez Lara, for sharing his expertise on lipidprotein binding assays and for teaching me the DLS method.

I am very thankful to Dr. Andrew Woehler, for introducing me to the FRAP method, and for Markus Niebert for providing access to the confocal microscope at the department of Physiology for FRAP measurements. I also thank Prof. Dr. Carsten Lüder for allowing me to use the confocal microscope at the Medical Microbiology department.

I thank all the members of the Cellular and Molecular Immunology department for their support, advice and assistance. I thank Dr. Michael Engelke for introducing me to the lab in the beginning of my $\mathrm{PhD}$, for sharing his expertise and ideas. I thank Ines Heine and Gabriele Sonntag for excellent technical assistance, and Ingrid Teuteberg and Annika Schindler for organizational support. Special thanks to Julius for interesting discussions and exchanging ideas, and to Christopher for providing the SLP65 knock out cell line for my experiments. I thank Caren, Kathrin, Christoffer, Kanika, Kai, Henrike and Wiebke for their support, friendship and for the fun times we had during lunch and coffee breaks, as well as outside of the lab. 
I would like to thank my other friends and family for encouraging and supporting me throughout my graduate studies. Thank you, Maria, Metin, Ömer, Avani, Ingrid and Myro for all the good times during my PhD years. Our occasional meetings for drinks, brunch, barbeque, culture nights, going to the swimming pool, playing board games etc. gave me energy throughout my $\mathrm{PhD}$, and these are the times I will never forget! Thank you, Oana, for staying in touch, for your moral support during our long conversations on the phone and your occasional visits.

I thank my parents and sisters, as well as my grandparents and my aunt, for supporting me morally and financially throughout my studies abroad; and for always being so close, while being thousands of kilometers away.

Dear Jonas, I cannot thank you enough for your support! Thank you for your patience and energy, for helping me to deal with frustration, for your helpful suggestions and proofreading of this thesis, for distracting me from work when needed, and for making me happy every day throughout the last years. And special thanks to my future child for helping me to stay calm during the writing of this thesis. 


\section{Curriculum Vitae}

\section{Sona Pirkuliyeva}

Personal details

Date of birth:

10.10.1989

Place of birth:

Ashgabat, Turkmenistan

Contact details:

Theaterstraße 3, 37073 Göttingen

(+49) 17684350506

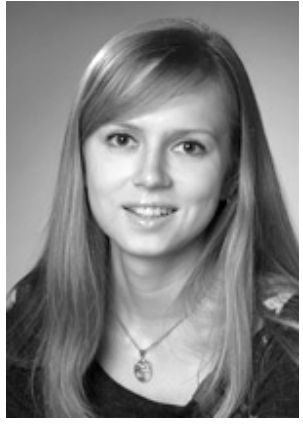

sonapirkuliyeva@yahoo.com

\section{Education}

10/2011-present

10/2011-present

PhD thesis project, Georg-August University of Goettingen, dept. of Cell. \& Mol. Immunology, thesis title "Structural and functional investigation of the primary transducer module of the B cell antigen receptor", supervised by Prof. Dr. Jürgen Wienands.

PhD program "Molecular Biology", Göttingen Graduate School for Neurosciences, Biophysics and Molecular Biology (GGNB).

10/2011-03/2012 MSc thesis project, Georg-August University Göttingen, dept. of Cellular and Molecular Immunology, thesis title "The role of $C$ type lectin 17A in B cell activation", supervised by Prof. Dr. Jürgen Wienands, completed with grade 1.3.

09/2010 - 09/2011 MSc studies, Msc/PhD program "Molecular Biology", International Max-Planck Research School, Georg-August University Göttingen, completed with the grade B (very good) and qualified for direct admission to the PhD program.

10/2006-07/2010 BSc studies at the Middle East Technical University, Ankara, Turkey, dept. of Molecular Biology and Genetics, graduated with CumGPA 3.52 (of 4.0 max), High Honor Student.

$2001-2006$

High School education - Turkmen-Turkish Girls High School, Ashgabat, Turkmenistan, graduated with the grade 5.0 (of 5.0 max).

Teaching experience

04/2013-06/2013

Supervision of practical training students, Medical University Göttingen, dept. of Cellular and Molecular Immunology

$2011 \& 2012$

Tutorials for MSc students from "Molecular Biology" program, topics "Immunology", "Signal Transduction", "Transcription".

\section{Administrative experience}

$2012 \& 2013$

Organization of the International PhD student symposium "Horizons in Molecular Biology" 
Language skills

$\begin{array}{ll}\text { German } & \text { Advanced (C1) } \\ \text { English } & \text { Fluent (C2) } \\ \text { Turkish } & \text { Fluent (C2) } \\ \text { French } & \text { Basic (A1) } \\ \text { Russian } & \text { Native } \\ \text { Turkmen } & \text { Native }\end{array}$

Stipends \& Awards

$03 / 2014$

Best Poster presentation, $1^{\text {st }}$ prize, awarded by German Society for Immunology, $10^{\text {th }}$ Spring School on Immunology, Ettal

$10 / 2012$ \& 09/2013 Traveling grants of the GGNB for attending $4^{\text {th }}$ Autumn School in Immunology (Bad Schandau, 10/2012), International Symposium on the Regulators of the Humoral Immune response (10/2012, Erlangen), $4^{\text {th }}$ International Symposium Regulators of Adaptive Immunity (Erlangen, 09/2013)

09/2011 - 08/2013 Stipend of the Excellence Foundation for the promotion of the MaxPlanck Society

09/2007-07/2010 Nippon Foundation Scholarship for Central Asian University students in Turkey

\section{Selected conferences}

$09 / 2014$

Oral presentation, selected talk "Assembly of SLP65 at vesicular membranes for the initiation of BCR signaling", $44^{\text {th }}$ Annual Meeting of the German Society for Immunology, Bonn

$03 / 2014$

Poster presentation "Assembly of signaling proteins on vesicular platforms for the initiation of humoral immune responses", $10^{\text {th }}$ Spring School on Immunology, Ettal and $12^{\text {th }}$ B cell Forum, Krickenbeck.

$10 / 2012$

Oral presentation "The role of CLEC17a in B cell activation" $4^{\text {th }}$ Autumn School Current Concepts in Immunology, Bad Schandau, Germany

M. Engelke, S. Pirkuliyeva*, J. Kühn*, L. Wong, J. Boyken, N. Herrmann, S. Becker, C. Griesinger, J. Wienands. Macromolecular assembly of the adaptor SLP-65 at intracellular vesicles in resting B cells, Science Signalling 7 (339), ra. 79 